Ergod. Th. \& Dynam. Sys. (1987), 7, 415-462

Printed in Great Britain

\title{
Diffeomorphisms on surfaces with a finite number of moduli
}

\author{
W. DE MELO AND S.J. VAN STRIEN
}

IMPA, Estrada Dona Castorina, 110, Rio de Janeiro, Brazil; Department of Mathematics, Techniche Hogeschool Delft, 2628 BL Delft, Julianalaan, 134 Holland

(Received 14 January 1986)

Abstract. This paper generalises the well known structural stability theorem which says that a diffeomorphism is structurally stable if it is Axiom $A$ and if all invariant manifolds are transversal to each other.

If these transversality conditions are not satisfied then the diffeomorphism not only fails to be stable, but also this gives rise to the appearance of moduli. That is, one needs several real parameters to parameterise all conjugacy classes of nearby diffeomorphisms. (The minimum number of parameters needed is called the number of moduli).

Here we deal with diffeomorphisms on two dimensional manifolds, whose asymptotic dynamics are well understood (the class of Axiom $\boldsymbol{A}$ diffeomorphisms). The main result characterises those Axiom $A$ diffeomorphisms which have a finite number of moduli. This result can be regarded as a generalisation of the structural stability theorem. From the proofs it follows that the dynamics of these diffeomorphisms can also be well understood.

In the proof of our main theorem we need certain invariant foliations to be quite smooth. In an appendix we prove a differentiable version of the Lambda Lemma.

One of the purposes of the theory of Dynamical Systems is to understand the orbit structure of diffeomorphisms. Here we say that two diffeomorphisms $f$ and $g$ have the same orbit structure if they are conjugate that is, if there is a homeomorphism $h$ of the ambient manifold such that $h f=g h$. Clearly this defines an equivalence relation $\sim$ on the space of diffeomorphisms. Since the dynamics of many diffeomorphisms is very complicated and sensitive to perturbations (of the diffeomorphisms) we cannot hope to understand the space of conjugacy classes in general. However this space is very neat in neighbourhoods of many conjugacy classes. This paper is in the direction of characterising all diffeomorphisms representing these conjugacy classes. One may measure the degree of complexity of this local structure by looking at the local dimensions of this space. This dimension we call the number of moduli. This number is defined as follows. If a diffeomorphism $f$ is structurally stable or if there are at most a countable number of different conjugacy classes in a neighbourhood $U$ of $f$ then we say that the number of moduli is zero. If a sufficiently small neighbourhood $U$ contains a countable number of $k$-parameter $C^{1}$ families of diffeomorphisms such that each diffeomorphism in $U$ is conjugate 
to at least one diffeomorphism from these families, then $f$ has a finite number of moduli. The minimum number $k$ of parameters needed is the number of moduli (or the modality of $f$ ).

Here we deal with diffeomorphisms on two dimensional manifolds whose asymptotic dynamics is well understood. In fact we consider the set $\mathscr{A}$ of diffeomorphisms satisfying Axiom A and the no cycle condition. Such diffeomorphisms are $\Omega$-stable, namely, the dynamics of the non-wandering set does not change with small perturbations [Sm1], [Sm2]. However the intersection pattern of invariant manifolds is the same for conjugate diffeomorphisms. Transversality conditions of stable and unstable manifolds are needed to have structural stability [R]. If these transversality conditions are not satisfied then the diffeomorphism not only fails to be stable but this also gives rise to the appearance of moduli [P2], [NPT], [MPS], [S1], [S2]. However even in this case the diffeomorphism may still have finite modality [Me2], [MP], [S2]. The purpose of this paper is to characterise the class $\mathcal{M}$ of diffeomorphisms in $\mathscr{A}$ having finite modality.

Our results provide a much more precise description of the conjugacy classes in a sufficiently small neighbourhood $\mathcal{N}$ of a diffeomorphism $f$ in $\mathcal{M}$. One has that $\mathcal{N}$ is a countable union of submanifolds $\mathscr{F}_{i}$. Each $\mathscr{F}_{i}$ has finite codimension and contains a finite dimensional submanifold $S_{i}$. Moreover there exists a differentiable fibration $\pi_{i}: \mathscr{F}_{i} \rightarrow S_{i}$ so that all diffeomorphisms in the same fiber are conjugate. Furthermore each $S_{i}$ contains a dense subset $T_{i}$ such that no two distinct diffeomorphisms in $T_{i}$ are conjugate to each other. The codimension of $\mathscr{F}_{i}$ is uniformly bounded and the maximum of all the dimensions of $S_{i}$ is the modality of $f$.

\section{Statement of results}

From now on let $M$ be a compact, $C^{\infty}$, two-dimensional manifold without boundary and $\operatorname{Diff}^{\infty}(M)$ be the set of $C^{\infty}$ diffeomorphisms on $M$ with the $C^{\infty}$ topology.

We denote by $\mathscr{A} \subset \operatorname{Diff}^{\infty}(M)$ the set of diffeomorphisms satisfying Axiom A and the no-cycle condition. Recall that $f \in \operatorname{Diff}^{\infty}(M)$ satisfies Axiom A if the nonwandering set $\Omega(f)$ is hyperbolic and the periodic orbits are dense in $\Omega(f)$, [Sm1]. If $f$ satisfies Axiom A then $\Omega(f)$ is a union of finitely many closed invariant sets $\Omega_{i}$ 's, called basic sets, such that $f$ has an orbit dense in each $\Omega_{i}$. Finally we say that $f$ satisfies the no-cycle condition if there are no basic sets $\Omega_{1}, \ldots, \Omega_{n}, \Omega_{n+1}=\Omega_{1}$ with $W^{u}\left(\Omega_{i}\right) \cap W^{s}\left(\Omega_{i+1}\right) \neq \varnothing$. Here $W^{u}\left(\Omega_{i}\right)$ (resp. $W^{s}\left(\Omega_{i}\right)$ ) is the set of points whose $\alpha$-limit set (resp. $\omega$-limit set) is contained in $\Omega_{i}$. We recall that if $f \in \mathscr{A}$ then $f$ is $\Omega$-stable [Sm2]. The main result of this paper is the characterisation of the set $M \subset \mathscr{A}$ of diffeomorphisms having finite moduli. Here the notion of modulus is defined as in the introduction. Remark that if $f$ has $k$ moduli then $f$ has a neighbourhood $U$ such that each $g$ in $U$ has modality at most $k$. In particular the set of diffeomorphisms in $\mathscr{A}$ with finite modality is open.

Let $M \subset \mathscr{A}$ be the set of diffeomorphisms satisfying the conditions below:

(1) if $x, y \in \Omega(f)$ are such that $W^{u}(x)$ is not transversal to $W^{s}(y)$ then the basic sets containing $x$ and $y$ are trivial (i.e. consist of periodic orbits); 
(2) there is only a finite number of orbits of non-transversal intersections between stable and unstable manifolds and the contact between these manifolds along each of these orbits is of finite order;

(3) if $p, q \in \operatorname{Per}(f)$ are such that $W^{u}(p)$ has an orbit of non-transversal intersection with $W^{s}(q)$ then the number of orbits in $W^{s}(p)$ (resp. in $W^{u}(q)$ ) belonging to some unstable (resp. stable) manifolds of periodic saddle points of $f$ is finite;

(4) if $x$ is a point of non-transversal intersection of $W^{u}(p)$ and $W^{s}(q)$ then there exists an arc $\Sigma$ transversal to $W^{u}(p)$ at $x$ such that no connected component of $\Sigma-\{x\}$ contains points of both stable and unstable manifolds of saddles;

(5) if $W^{u}(p)$ has a point of non-transversal intersection with $W^{s}(q)$, and $W^{u}(q)$ has a point of non-transversal intersection with $W^{s}(r)$, then there is no saddle point of $f$ whose unstable manifold (resp. stable manifold) intersects $W^{s}(p)$ (resp. $W^{u}(r)$ ).

Remark. It is well known that every diffeomorphism in $\mathscr{A}$ which satisfies the transversality conditions on invariant manifolds is structurally stable [Rb1]. The class $\mathcal{M}$ relaxes these conditions. The following theorem generalizes this structural stability result.

MAIN TheOREM. If $f \in \operatorname{Diff}^{\infty}\left(M^{2}\right)$ is in $\mathscr{A}$ then $f$ has finite modality if and only if $f \in \mathcal{M}$.

Remark. As in [P1] it is not hard to show that if $f \in \operatorname{Diff}^{\infty}\left(M^{2}\right)$ satisfies the Axiom $A$ condition and $f$ has cycles then $f$ has infinite modality.

\section{Existence of moduli}

Let $M$ be a compact $C^{\infty}$ manifold of dimension two and $f: M \rightarrow M$ be a $C^{\infty}$ diffeomorphism having a pair of periodic points $p$ and $q$ such that the unstable manifold of $p$ has an orbit of non-transversal intersection with the stable manifold of $q$. In this section we will construct all conjugacy invariants generated by the existence of these tangencies. We will use these conjugacy invariants to prove the following.

THEOREM. If $f \in \mathscr{A}$ has finite modality then $f \in \mathcal{M}$.

Later in $\S \S 3,4$ and 5 we will prove that two diffeomorphisms in $\mathcal{M}$, having an equivalent intersection pattern of stable and unstable manifolds, are conjugate if they have the same conjugacy invariants.

2(a). Some technical lemmas. In order to show the existence of moduli we will have to compare metrics induced on $M$ by two $C^{1}$ coordinate systems. So we start by describing some properties of $C^{r}$ metrics. By this we mean metrics which are induced by $C^{r}$ Riemannian structures. We say that $d: M \times M \rightarrow \mathbb{B}$ is a $C^{r}$ metric, $0 \leq r \leq \infty$, on $M$ if there exists a $C^{r}$ Riemannian structure $g$ on $M$ such that $d(x, y)$ is given by the infimum of the lengths of all paths that connect $x$ and $y$. In formulae:

$$
\begin{aligned}
& d(x, y)=\inf \left\{l_{g}(\gamma) ; \gamma:[0,1] \rightarrow M\right. \text { is a piecewise } \\
& \left.\qquad C^{1} \text { curve with } \gamma(0)=x \text { and } \gamma(1)=y\right\} .
\end{aligned}
$$


Here

$$
l_{g}(\gamma)=\int_{0}^{1} \sqrt{g\left(\gamma^{\prime}(t), \gamma^{\prime}(t)\right)} d t
$$

The distance from a point $x$ to a set $S$ will be denoted by $d(x, S)=\inf \{d(x, y) ; y \in S\}$. It will be convenient to use the following notation. For sequences $\alpha_{i}, \beta_{i}$ of real numbers we will write

$$
\begin{array}{ll}
\alpha_{i} \sim \beta_{i} \quad \text { if } \quad\left|\frac{\alpha_{i}}{\beta_{i}}\right| \text { is bounded and bounded away from zero, } \\
\alpha_{i}=\beta_{i} \quad \text { if } \quad \frac{\alpha_{i}}{\beta_{i}} \text { converges to } 1 .
\end{array}
$$

LEMMA 2.1. Let $d: \mathbb{R}^{n} \times \mathbb{R}^{n} \rightarrow \mathbb{R}$ be a $C^{0}$ metric on $\mathbb{R}^{n}$ induced by the Riemannian structure $g$. Let $d_{0}$ be the metric induced by the constant Riemannian structure $g_{0}$ which coincides with $g$ at 0 . If $S \subset \mathbb{R}^{n}$ contains 0 and $x_{i} \in \mathbb{R}^{n}-S$ converges to zero, then $d\left(x_{i}, S\right) \simeq d_{0}\left(x_{i}, S\right)$.

Proof. Since $g$ is continuous, for every $\rho>0$ there are positive constants $c_{\rho}, c_{\rho}^{\prime}$ such that

$$
c_{\rho} \sqrt{g((0, v),(0, v))} \leq \sqrt{g((x, v),(x, v))} \leq c_{\rho}^{\prime} \sqrt{g((0, v),(0, v))}
$$

for every $v \in \mathbb{R}^{n}$ and every $x \in \mathbb{R}^{n}$ with $\|x\|<\rho$. Clearly we can take $c_{\rho}$ and $c_{\rho}^{\prime}$ arbitrarily near 1 by taking $\rho$ sufficiently small. From (2.1) we have that if $\gamma$ is a piecewise $C^{1}$ curve contained in the ball with centre 0 and radius $\rho$ then

$$
c_{\rho} l_{g_{0}}(\gamma) \leq l_{g}(\gamma) \leq c_{\rho}^{\prime} l_{g_{0}}(\gamma),
$$

where $g_{0}$ is the constant Riemannian structure, i.e. $g_{0}((x, v),(x, w))=g((0, v),(0, w))$ for all $x, v, w \in \mathbb{R}^{n}$. Hence, if $\bar{\rho}>0$ is sufficiently small then

$$
c_{\rho} d_{0}(x, y) \leq d(x, y) \leq c_{\rho}^{\prime} d_{0}(x, y)
$$

for all $x, y \in \mathbb{R}^{n}$ with $\|x\|,\|y\| \leq \bar{\rho}$. Therefore for $\overline{\bar{\rho}}>0$ sufficiently small we have that

$$
c_{\rho} d_{0}(x, S) \leq d(x, S) \leq c_{\rho}^{\prime} d_{0}(x, S)
$$

for all $x \in \mathbb{R}^{n}$ with $\|x\| \leq \bar{\rho}$. This proves the lemma.

LEMMA 2.2. Let $S \subset \mathbb{R}^{n}$ be a codimension one $C^{1}$ submanifold containing 0 and $d_{j}$, $j=1,2$ be $C^{0}$ metrics on $\mathbb{R}^{n}$. Then there exists a positive real number $\lambda$ such that $d_{1}\left(x_{i}, S\right) \simeq \lambda d_{2}\left(x_{i}, S\right)$ for any sequence $x_{i} \in \mathbb{R}^{n}-S$ converging to $0 \in \mathbb{R}^{n}$.

Proof. By taking $C^{1}$ coordinates on $\mathbb{R}^{n}$ we may assume that $S$ is a hyperplane. By lemma 2.1 we can assume that $d_{1}$ and $d_{2}$ are induced by Riemannian structures which are constant with respect to this coordinate system. From these observations the lemma follows easily.

Remark. If $\tilde{S}$ is another $C^{1}$ codimension one submanifold tangent to $S$ at 0 and $x_{i} \in \mathbb{R}^{n}-\tilde{S}$ converges to 0 then $d_{1}\left(x_{i}, \tilde{S}\right) \simeq \lambda d_{2}\left(x_{i}, \tilde{S}\right)$, where $\lambda$ is the same constant as for $S$. This follows from the proof of the lemma and the fact that we can find a pair of $C^{1}$ diffeomorphisms $\varphi_{1}, \varphi_{2}:\left(\mathbb{R}^{n}, 0\right) \varsigma$ such that $\varphi_{1}(S)=\varphi_{2}(\tilde{S})$ is a hyperplane and the derivative of $\varphi_{1} \circ \varphi_{2}^{-1}$ at 0 is the identity. 
Using $C^{0}$ metrics we can introduce the notion of contact of $C^{1}$ submanifolds. Definition. Let $x$ be a point of tangency of two $C^{1}$ submanifolds $S_{1}, S_{2} \subset M$. We say that $S_{1}$ has a contact of order $n$ with $S_{2}$ at $x$ if for some metric $d$ on $M$ the limit:

$$
\lim _{\substack{w \rightarrow x \\ w \in S_{1}}} \frac{d\left(w, S_{2}\right)}{[d(w, x)]^{n}}
$$

exists and is positive. If this limit is infinite we say that the contact is less than $n$. If the limit is zero for all $n$ we say that the contact is infinite.

Remark. From lemmas 2.1 and 2.2 it follows that the above definition is independent of the metric. Notice also that the contact may not exist. However if there is a $C^{1}$ coordinate system $\varphi$ on a neighbourhood of $x$ such that $\varphi\left(S_{1}\right)$ and $\varphi\left(S_{2}\right)$ are both $C^{\infty}$ submanifolds of $\mathbb{R}^{2}$ then the contact is either defined or it is infinite.

Definition. Let $p$ be a hyperbolic fixed point of a $C^{\infty} \operatorname{diffeomorphism} f: M \rightarrow M$. By a linearising metric at $p$ we mean a $C^{0}$ metric $d$ on a neighbourhood $U$ of $W^{s}(p) \cup$ $W^{u}(p)$ such that in a $C^{1}$ coordinate system in $U$ linearising $f, d$ coincides with the Euclidean metric.

Remark. If $\overline{W^{s}(p)} \cap \overline{W^{u}(p)}=\{p\}$, such a linearising metric always exists. In fact by a theorem of Hartman [Ha] (see also the appendix) there is a $C^{1}$ coordinate system in a neighbourhood of $p$ linearising $f$. If $\overline{W^{s}(p)} \cap \overline{W^{u}(p)}=\{p\}$, this coordinate system can be extended to a full neighbourhood of $W^{s}(p) \cup W^{u}(p)$. Thus we obtain a linearising metric at $p$. These metrics are not unique. However, since any $C^{1}$ diffeomorphism commuting with a linear contraction $l: \mathbb{R} \rightarrow \mathbb{R}$ is linear, it is easy to see that if $\tilde{d}$ is another linearising metric then the restrictions of $d$ and $\tilde{d}$ to each connected component of $W^{s}(p) \cup W^{u}(p)-\{p\}$ differ only by a multiplicative constant.

The following two lemmas will be used repeatedly.

LEMMA 2.3. Let $p$ be a hyperbolic fixed point of saddle type of a $C^{2}$ diffeomorphism $f: M \rightarrow M$. Let $x \in W^{u}(p)-\{p\}, d$ be $a C^{0}$ metric on $M$ and $\alpha$ be the contracting eigenvalue of $d f_{p}$. For any sequence $x_{i} \rightarrow x$ we have:

(i) if there exists a sequence $n(i) \rightarrow \infty$ such that $f^{-n(i)}\left(x_{i}\right)$ converges to a point $z \in W^{s}(p)$, then $d\left(x_{i}, W^{u}(p)\right) \simeq c d(z, p)|\alpha|^{n(i)}$ for some constant $c$ which depends on $x, z$ and $d$ but not on the sequence; if $d$ is a linearising metric then $c$ is independent of $x$ and $z$;

(ii) if $d\left(x_{i}, W^{u}(p)\right) \simeq c|\alpha|^{n(i)}$ for some constant $c>0$ and some sequence $n(i) \rightarrow \infty$ then the sequence $f^{-n(i)}\left(x_{i}\right)$ has precisely one limit point if $\alpha>0$ and two limit points if $\alpha<0$. These limit points are contained in $W^{s}(p)$.

Proof. It is clear that both statements hold for a linearising metric. Therefore the lemma follows from lemma 2.2 .

Definition. Let $x$ be a one sided tangency (for example a quadratic tangency or a tangency of even order) between $W^{u}(p)$ and $W^{s}(q)$, where $p$ and $q$ are hyperbolic fixed points of $f$. Let $y \in W^{u}(q)$. We say that a sequence $x_{i} \rightarrow x$ is nice with respect to the pair $(x, y)$ if (i) $f^{i}\left(x_{i}\right)$ converges to $y$; (ii) $d\left(x_{i}, W^{u}(p)\right) \simeq d\left(x_{i}, W^{s}(q)\right)$. 
We will now prove that the property of being a nice sequence is preserved under a topological conjugacy.

LEMMA 2.4. Let h be a conjugacy between $f$ and $\bar{f}$. Then $x_{i} \rightarrow x$ is a nice sequence with respect to $(x, y)$ if and only if $h\left(x_{i}\right)$ is a nice sequence with respect to $(h(x), h(y))$.

Proof. We have two cases to consider: (a) $y$ is accumulated by the iterates of any small interval in $W^{u}(p)$ which contains $x$; (b) case (a) does not occur. In both cases it is easy to see that we have the following characterisation of nice sequences. The sequence $x_{i} \rightarrow x$ is nice with respect to $(x, y)$ if (i) $f^{i}\left(x_{i}\right) \rightarrow y$; (ii) given sequences of integers $n(j) \rightarrow \infty$ and $i(j) \rightarrow \infty$ such that $f^{-n(j)}\left(x_{i(j)}\right)$ converges to $z \in W^{s}(p)$ then for any other sequence $x_{i}^{\prime} \rightarrow x$ such that $f^{i}\left(x_{i}^{\prime}\right) \rightarrow y$ we have:

$$
\left.\begin{array}{ll}
\lim \sup d\left(f^{-n(j)}\left(x_{i(j)}^{\prime}\right), p\right) \leq d(z, p) & \text { in case (a), } \\
\lim \inf d\left(f^{-n(j)}\left(x_{i(j)}^{\prime}\right), p\right) \geq d(z, p) & \text { in case (b). }
\end{array}\right\}
$$

In fact let $x_{i}$ be nice with respect to $(x, y)$. Then $d\left(x_{i}, W^{u}(p)\right) \simeq d\left(x_{i}, W^{s}(q)\right)$ and $f^{i}\left(x_{i}\right) \rightarrow y$. If for some other sequence $x_{i}^{\prime}$ we also have $f^{i}\left(x_{i}^{\prime}\right) \rightarrow y$, then by lemma 2.3 one has $d\left(x_{i}, W^{s}(q)\right) \simeq d\left(x_{i}^{\prime}\right.$. $\left.W^{s}(q)\right)$. Moreover since $W^{u}(p)$ and $W^{s}(q)$ have one-sided tangencies

$$
\begin{array}{ll}
d\left(x_{i}^{\prime}, W^{u}(p)\right) \leq d\left(x_{i}^{\prime}, W^{s}(q)\right) & \text { in case (a), } \\
d\left(x_{i}^{\prime}, W^{u}(p)\right) \geq d\left(x_{i}^{\prime}, W^{s}(q)\right) & \text { in case (b). }
\end{array}
$$

Hence the limit points of $\left\{d\left(x_{i}^{\prime}, W^{u}(p)\right) / d\left(x_{i}, W^{u}(p)\right)\right\}$ are at most 1 in case (a), and at least 1 in case (b). From this and lemma 2.3 one deduces (*). Similarly (*) implies that $x_{i}$ is a nice sequence. Since $W^{s}(p)$ has dimension one, it follows easily that these characterisations are preserved under conjugacy.

2(b). Construction of moduli. Now we will construct all conjugacy invariants arising from non-transversal intersection of invariant manifolds of periodic points. To simplify the exposition we will assume that all periodic points involved are in fact fixed points. The general case is treated in the same way by looking at appropriate iterates of the diffeomorphism. The next lemma introduces the first conjugacy invariant found in [P2] and shows the rigidity of the conjugacy if this invariant is irrational as in [Me2].

LEMMA 2.5. Let $x$ (resp. $\bar{x}$ ) be a tangency of even order between $W^{u}(p)$ and $W^{s}(q)$ (resp. $W^{u}(\bar{p})$ and $W^{s}(\bar{q})$ ) where $p$ and $q$ (resp. $\bar{p}$ and $\bar{q}$ ) are hyperbolic fixed points of the $C^{2}$ diffeomorphism $f$ (resp. $\bar{f}$ ). Let $\alpha$ (resp. $\bar{\alpha}$ ) be the contracting eigenvalue of $d f_{p}\left(\right.$ resp. $\left.d \bar{f}_{\bar{p}}\right)$ and $\beta$ (resp. $\left.\bar{\beta}\right)$ be the expanding eigenvalue of $d f_{q}$ (resp. $\left.d \bar{f}_{\bar{q}}\right)$. Let $h$ be a conjugacy between $f$ and $\bar{f}$ with $h(p)=\bar{p}, h(q)=\bar{q}$ and $h(x)=\bar{x}$. Then we have the following properties:

$$
\frac{\log |\alpha|}{\log |\beta|}=\frac{\log |\bar{\alpha}|}{\log |\bar{\beta}|}
$$

(2) Let $d_{p}$ be a linearising metric at $p$ and $d_{q}$ be a linearising metric at $q$. If $\log |\alpha| / \log |\beta|$ is irrational then

$$
\frac{d_{\bar{p}}(h(z), \bar{p})}{\left[d_{p}(z, p)\right]^{\log |\tilde{\alpha}| / \log |\alpha|}}
$$


is constant in each connected component of $W^{s}(p)-\{p\}$, and

$$
\frac{d_{\bar{q}}(h(w), \bar{q})}{\left[d_{q}(w, q)\right]^{\log |\bar{\beta}| / \log \beta \mid}} .
$$

is constant in each connected component of $W^{u}(q)-\{q\}$.

Proof. (1) Let $x_{i} \rightarrow x$ be a nice sequence with respect to $(x, y)$ where $y \in W^{u}(q)$. Hence by lemma $2.4 \bar{x}_{i}=h\left(x_{i}\right)$ is a nice sequence with respect to $(h(x), h(y))$. Choose subsequence $i(j)$ and $n(j)$ such that $f^{-n(j)}\left(x_{i(j)}\right)$ converges to a point $z \in W^{s}(p)$. From lemma 2.3 it follows that

$$
c d_{p}(z, p)|\alpha|^{n(j)} \simeq d\left(x_{i(j)}, W^{u}(p)\right) \simeq d\left(x_{i(j)}, W^{s}(q) \simeq c^{\prime} d_{q}(y, q)|\beta|^{i(j)} .\right.
$$

Hence $i(j) / n(j) \simeq \log |\alpha| / \log |\beta|$. Since $h$ is a conjugacy it follows also that $i(j) / n(j) \simeq \log |\bar{\alpha}| / \log |\bar{\beta}|$. Thus

$$
\frac{\log |\alpha|}{\log |\beta|}=\frac{\log |\bar{\alpha}|}{\log |\bar{\beta}|}
$$

(2) Let $x_{i}$ be nice with respect to $(x, y)$. From the first part of the proof it follows that for any $i(j), n(j) \rightarrow \infty$,

$$
d_{p}\left(f^{-n(j)}\left(x_{i(j)}\right), p\right) \approx \Delta_{x} \cdot d_{q}(y, q) \cdot|\beta|^{i(j)} \cdot|\alpha|^{-n(j)},
$$

where $\Delta_{x}=c^{\prime} / c$. It follows that for any $z$ in one component of $W^{s}(p)-\{p\}$ there exists $i(j), n(j)$ such that $f^{-n(j)}\left(x_{i(j)}\right) \rightarrow z$, since $\log |\alpha| / \log |\beta|$ is irrational. Because the same holds for $\bar{f}$ one has

$$
\frac{d_{\bar{p}}(h(z), \bar{p})}{\left(d_{p}(z, p)\right)^{\delta}}=\frac{\Delta_{\bar{x}}}{\left(\Delta_{x}\right)^{\delta}} \cdot \frac{d_{\bar{q}}(\bar{y}, \bar{q})}{\left(d_{q}(y, q)\right)^{\delta}},
$$

where $\delta=\log |\bar{\alpha}| / \log |\alpha|=\log |\vec{\beta}| / \log |\beta|$. Since the right hand side of this equation is independent of $z$, the result follows.

Remarks. (1) From the second part of lemma 2.5 it follows that the restriction of $h$ to $W^{s}(p)-\{p\}$ and to $W^{u}(q)-\{q\}$ is a $C^{1}$ diffeomorphism.

(2) The restriction of $h$ to each component of $W^{s}(p)-\{p\}$ and $W^{u}(q)-\{q\}$ is determined by the image of one point. This is the rigidity of the conjugacy we have mentioned before.

COROLLARY 1. Each orbit of tangency between stable and unstable manifolds of periodic orbits gives rise to at least one modulus condition.

Proof. If $f$ has $k$ orbits of tangency, then arbitrarily near $f$ there is a diffeomorphism $\bar{f}$ with at least $k$ orbits of tangency of even order. Let $W^{u}(p)$ and $W^{s}(q)$ have tangencies of even order along $r$ orbits $O\left(x_{1}\right), \ldots, O\left(x_{r}\right)$. Then from the proof of lemma 2.5 (using the same notation) one obtains the following $r$ conditions:

$$
\frac{\log |\alpha|}{\log |\beta|}=\frac{\log |\bar{\alpha}|}{\log |\bar{\beta}|} \text { and } \frac{\left(\Delta x_{i}\right)^{\delta}}{\Delta_{\bar{x}_{i}}}=\frac{\left(\Delta_{x_{j}}\right)^{\delta}}{\left(\Delta_{\bar{x}_{j}}\right)} \text {. }
$$

From this corollary 1 follows.

Corollary 2. If $f \in \mathscr{A}$ has finite moduli then satisfies conditions (1), (2), (3) and (5) of $\S 1$. 
Proof. If $f \in \mathscr{A}$ does not satisfy (1), (2), (3) or (5) then for any $k \in \mathbb{N}$ there exists a diffeomorphism $f$ arbitrarily near $f$ satisfying one of the following properties:

(a) $\bar{f}$ has at least $k$ orbits of tangency between stable and unstable manifolds of periodic orbits;

(b) there exist saddles $\bar{p}, \bar{q}$ so that $W^{u}(\bar{p})$ and $W^{s}(\bar{q})$ are tangent and so that there exist an infinite number of orbits of intersections of invariant manifolds of saddles with $W^{s}(\bar{p})$ or $W^{u}(\bar{q})$. In the latter case $\bar{f}$ has modality at least $k$ because of remark 2 above. In the former case $\bar{f}$ has modality $\infty$ because of corollary 1 . Since this holds for any $k$ the modality of $f$ is $\infty$.

LEMMA 2.6. Let $\bar{f}$ and $f$ be as in lemma 2.5 with $\log |\alpha| / \log |\beta|$ irrational. Let $r$ (resp. $\bar{r}$ ) be a hyperbolic fixed point of $f$ (resp. $\bar{f}$ ) of saddle type whose stable manifold intersects transversally the unstable manifold of $q$ (resp. $\bar{q}$ ). If $h$ is a conjugacy between $f$ and $\bar{f}$ then the expanding eigenvalue of $d f(r)$ is equal to the expanding eigenvalue of $d \bar{f}(\vec{r})$.

Proof. Let $d$ (resp, $\bar{d}$ ) be $C^{0}$ metrics as before. Let $y_{i} \in W^{u}(q)$ be a sequence converging to $y \in W^{u}(q) \cap W^{s}(r)$ and such that $f^{i}\left(y_{i}\right)$ converges to a point $w \in$ $W^{u}(r)$. Since $h \mid W^{u}(q)-\{q\}$ is $C^{1}$, there exists a constant $c_{1}>0$ such that $\bar{d}\left(h\left(y_{i}\right), h(y)\right) \simeq c_{1} d\left(y_{i}, y\right)$. If $\gamma$ is the expanding eigenvalue of $d f(r)$ we have, by lemma 2.3,

$$
d\left(y_{i}, W^{s}(r)\right) \simeq c_{2} d(w, r)|\gamma|^{-i}
$$

for some constant $c_{2}>0$. Similarly

$$
\bar{d}\left(h\left(y_{i}\right), W^{s}(\bar{r})\right) \approx \bar{c}_{2} \bar{d}(\bar{w}, \bar{r})|\bar{\gamma}|^{-i} .
$$

From these equations we have $|\gamma|=|\bar{\gamma}|$. Clearly this implies $\gamma=\bar{\gamma}$.

Remark. Similarly to the above situation we have that the contracting eigenvalue at each saddle point whose unstable manifold intersects $W^{s}(p)$ transversally is a conjugacy invariant.

Now we will introduce some other conjugacy invariants which arise in the following situation: there are three fixed points $p, q, r$ such that $W^{u}(p)$ has a point $x$ of tangency with $W^{s}(q)$ of order $2 n$ and $W^{u}(q)$ has a point $y$ of tangency with $W^{s}(r)$ of order $2 m$. Take $C^{1}$ curves $\Sigma_{x}, \Sigma_{y}$ at $x, y$, transversal to $W^{s}(q)$ and $W^{u}(q)$ respectively. Take a neighbourhood $R$ of $q$ which contains $x$ and $y$, and on which $f$ has a linearising coordinate system. Let $\tilde{\Sigma}_{n, x}$ be the component of $f^{n}\left(\Sigma_{x}\right) \cap R$ containing $f^{n}(x)$, and similarly $\tilde{\Sigma}_{n, y}$ the component of $f^{-n}\left(\Sigma_{y}\right) \cap R$ containing $f^{-n}(y)$. There are four cases to consider: (see figure 2.1).

Case A. $\Sigma_{n, x} \cap W^{s}(r)$ and $\Sigma_{n, y} \cap W^{u}(p)$ do not accumulate to $y$ respectively $x$ as $n \rightarrow \infty$;

Case B. $\Sigma_{n, x} \cap W^{s}(r)$ does accumulate to $y$, but $\Sigma_{n, y} \cap W^{u}(p)$ does not accumulate to $x$;

Case C. $\Sigma_{n, x} \cap W^{s}(r)$ does not accumulate to $y$, but $\Sigma_{n, y} \cap W^{u}(p)$ does accumulate to $x$;

Case D. $\Sigma_{n, x} \cap W^{s}(r)$ and $\Sigma_{n, y} \cap W^{u}(p)$ do accumulate to $y$ respectively $x$. 

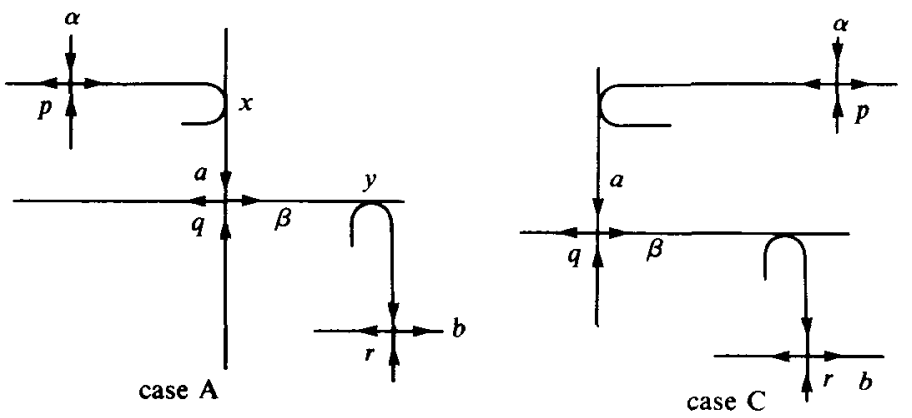

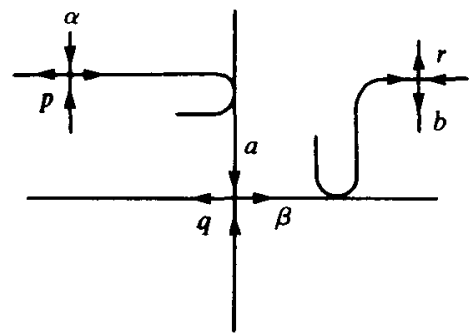

case B

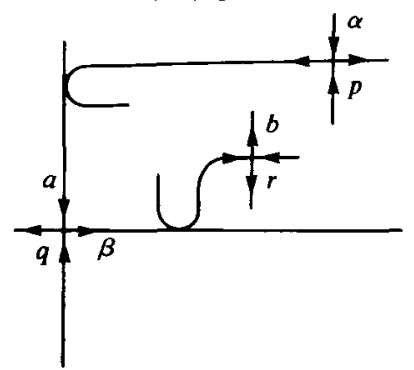

case D

Figure 2.1

LEMMA 2.7. Let $\bar{f}: M \rightarrow M$ be a diffeomorphism having the same intersection pattern of stable and unstable manifolds as $f$. Consider the following equations:

$$
\begin{array}{ll}
\text { (M1) } & \alpha=\bar{\alpha} \\
\text { (M2) } & \beta=\bar{\beta} \\
\text { (M3) } & a=\bar{a} \\
\text { (M4) } & b=\bar{b} \\
\text { (M5) } & \frac{Q_{x}[d(x, q)]^{2 n}}{d(y, q)}=\frac{Q_{\bar{x}}[\bar{d}(\bar{x}, \bar{q})]^{2 n}}{\bar{d}(\bar{y}, \bar{q})} \\
\text { (M6) } & \frac{Q_{y}[d(y, q)]^{2 m}}{d(x, q)}=\frac{Q_{\bar{y}}[\bar{d}(\bar{y}, \bar{q})]^{2 m}}{\bar{d}(\bar{x}, \bar{q})}
\end{array}
$$

where $\alpha$, a are contracting eigenvalues of $d f_{p}$ and $d f_{q} ; \beta, b$ are expanding eigenvalues of $d f_{q}, d f_{r} ; d$ is a linearising metric at $q$;

$$
Q_{x}=\lim _{\substack{w \in W^{s}(q) \\ w \rightarrow x}} \frac{d\left(w, W^{u}(p)\right)}{[d(w, x)]^{2 n}} ; \quad Q_{y}=\lim _{\substack{w \in W^{u}(q) \\ w \rightarrow y}} \frac{d\left(w, W^{s}(r)\right)}{[d(w, y)]^{2 m}} .
$$

If $\log |\alpha| / \log |\beta|$ and $\log |a| / \log |b|$ are irrational and $h$ is a conjugacy between $f$ and $\bar{f}$ such that $h(p)=\bar{p}, h(q)=\bar{q}, h(r)=\bar{r}, h(x)=\bar{x}$ and $h(y)=\bar{y}$ then the following conditions are satisfied:

Case A. Conditions (M1) to (M6).

Case B. Conditions (M1) to (M4) plus condition (M5).

Case C. Conditions (M1) to (M4) plus condition (M6).

Case D. Conditions (M1) to (M4). 
Proof. From lemma 2.5 we have that

and

$$
\frac{\log |\alpha|}{\log |\beta|}=\frac{\log |\bar{\alpha}|}{\log |\bar{\beta}|}
$$

$$
\frac{\log |a|}{\log |b|}=\frac{\log |\bar{a}|}{\log |\bar{b}|}
$$

Furthermore if these numbers are irrational we have that $h \mid W^{s}(q)-\{q\}$ and $h \mid W^{u}(q)-\{q\}$ are $C^{1}$ maps. We will show now that in all cases equalities (M1) to (M4) are satisfied. Take a sequence $x_{i} \in W^{s}(q)$ converging to the point of tangency $x$ and such that $f^{-n(i)}\left(x_{i}\right)$ converges to some point $z \in W^{s}(p)$. By lemma 2.3 we have that

$$
d\left(x_{i}, W^{u}(p)\right) \simeq c d(z, p)|\alpha|^{n(i)},
$$

where $c$ is a positive constant independent of the sequence. Now $W^{u}(p)$ and $W^{s}(q)$ have a tangency of order $2 n$ at $x$. Therefore if we consider a $C^{\infty}$ metric $d^{\prime}$ induced by a $C^{\infty}$ coordinate system in which $W^{u}(p)$ is a straight line and $W^{s}(q)$ is the graph of a homogeneous polynomial of degree $2 n$ then

$$
\frac{d^{\prime}\left(x_{i}, W^{u}(p)\right)}{\left[d^{\prime}\left(x_{i}, x\right)\right]^{2 n}} \rightarrow Q_{x}^{\prime}
$$

where $Q_{x}^{\prime}$ is a positive number. But since $d$ is a $C^{0}$ metric, lemma 2.2 tells us that $d\left(x_{i}, W^{u}(p)\right) / d^{\prime}\left(x_{i}, W^{u}(p)\right)$ converges to a positive constant which does not depend on the metric. Clearly the sequence $d\left(x_{i}, x\right) / d^{\prime}\left(x_{i}, x\right)$ also converges to a positive constant because $x_{i} \in W^{s}(q)$. This and (4) imply that

$$
\frac{d\left(x_{i}, W^{u}(p)\right)}{\left[d\left(x_{i}, x\right)\right]^{2 n}} \rightarrow Q_{x}
$$

and $Q_{x}>0$. Now $h \mid W^{s}(q)-\{p\}$ is $C^{1}$ so that

$$
d\left(x_{i}, x\right) \sim \bar{d}\left(h\left(x_{i}\right), \bar{x}\right) \text {. }
$$

Equations (3), (5) and (6) imply that $|\alpha|=|\bar{\alpha}|$. Hence $\alpha=\bar{\alpha}$. Similarly we prove that $b=\bar{b}$ and from (1) and (2) it follows that $\beta=\bar{\beta}$ and $a=\bar{a}$. If we are in case D then we are finished. So let us show that there are additional moduli in cases A, B, C. Let us prove that (M5) is satisfied in cases A and B. For that, take a sequence $x_{i} \rightarrow x$ nice with respect to $(x, y)$. Choose subsequences $i(j)$ and $n(j)$ such that $f^{-n(j)}\left(x_{i(j)}\right)$ converges to a point $z \in W^{s}(p)$. Since we are in case A or in case B we can take a sequence $z_{j} \in W^{s}(q)$ such that $f^{-n(j)}\left(z_{j}\right) \rightarrow z$. Since $\left\{x_{i}\right\}$ is nice with respect to $(x, y)$ and $d$ is a linearizing metric we have

So we have

$$
\begin{gathered}
d\left(x_{i(j)}, W^{s}(q)\right) \simeq d(y, q)|\beta|^{-i(j)}, \\
d\left(x_{i(j)}, W^{u}(p)\right) \simeq d\left(x_{i(j)}, W^{s}(q)\right) .
\end{gathered}
$$

$$
d\left(x_{i(j)}, W^{u}(p)\right) \simeq d(y, q)|\beta|^{-i(j)} .
$$

Since $f^{-n(j)}\left(x_{i(j)}\right)$ and $f^{-n(j)}\left(z_{j}\right)$ both converge to $z$ we have from lemma 2.3 that $d\left(x_{i(j)}, W^{u}(p)\right) \simeq d\left(z_{j}, W^{u}(p)\right)$. Finally we have equation $(5): d\left(z_{j}, W^{u}(p)\right) \simeq$ 
$Q_{x}\left\{d\left(z_{j}, x\right)\right\}^{2 n}$. This and (7) imply:

i.e.

$$
d(y, q) \cdot|\beta|^{-i(j)} \simeq Q_{x}\left\{d\left(z_{j}, x\right)\right\}^{2 n}
$$

$$
\frac{Q_{x}\{d(x, q)\}^{2 n}}{d(y, q)} \simeq\left\{\frac{d(x, q)}{d\left(z_{j}, x\right)}\right\}^{2 n} \cdot|\beta|^{-i(j)}
$$

Since $h \mid W^{s}(q)-\{q\}$ is linear and $d$ is a linearising metric we have

$$
\frac{d\left(z_{j}, x\right)}{d\left(h\left(z_{j}\right), h(x)\right)}=\frac{d(x, q)}{d(h(x), h(q))} \text {. }
$$

From this, $\beta=\bar{\beta}$, and (9) it follows that

is a topological invariant.

$$
\frac{Q_{x} \cdot\{d(x, q)\}^{2 n}}{d(y, q)}
$$

Similarly, we prove that in cases A and C condition (M6) is satisfied.

Kemark. It is not obvious that the numbers which appear in the modulus conditions (M5) and (M6) are independent of the choices made for the metric $d$. That these numbers are independent of the metrics is proved in $\S 3(\mathrm{~b})$. (In case $A$ this also follows from the conclusion of lemma (2.6)).

In $\S 5$ we shall show that the equations from lemma (2.6) are the only obstructions for constructing conjugacies between two diffeomorphisms as above.

Proof of the theorem. It remains to prove that if $f \in \mathscr{A}$ has finite modality then conditions 4 and 5 of $\S 1$ are satisfied. Notice that if $f$ has finite modality then the modality of any $g$ sufficiently near $f$ is at most equal to the modality of $f$. So we will prove that if $f \in \mathscr{A}$ does not satisfy condition 4 of $\S 1$ then $f$ can be approximated by diffeomorphisms with arbitrarily high modality. So let $f \in \mathscr{A}$ be a diffeomorphism having an orbit of non transversal intersection between $W^{s}(q)$ and $W^{u}(p)$. We may assume that all periodic points are in fact fixed points by considering a sufficiently high iterate of $f$. By taking a small perturbation of $f$ we may assume that $W^{u}(p)$ has a point $x$ of quasi-transversal (i.e. quadratic contact) intersection with $W^{s}(q)$. Now assume that condition 4 of $\S 1$ does not hold. Then any arbitrarily small arc $\Sigma$ at $x$ transversal to $W^{u}(p)$ contains unstable (stable) manifolds of saddles. Then, by taking a small perturbation of $f$ one can find a saddle $P$ (resp. $Q$ ) such that $W^{u}(P)\left(\right.$ resp. $\left.W^{s}(Q)\right)$ has an intersection with $W^{s}(p)$ (resp. $\left.W^{u}(q)\right)$. We also may assume that $W^{u}(P)$ (respectively $W^{s}(Q)$ ) contains a small interval $I$ (resp. $J$ ) which intersects transversally $W^{s}(p)$ (resp. $W^{u}(q)$ ) in a unique point (see figure 2.2). Now assume that condition 4 of $\S 1$ does not hold. Then $f^{k}(I)$ has an intersection with $J$ for any $k$ sufficiently big.

From the appendix it follows that we can construct an invariant $C^{1}$ unstable (resp. stable) foliation $\mathscr{F}_{p}^{u}$ (resp. $\mathscr{F}_{q}^{s}$ ), with a $C^{1}$ tangent line field, having $I$ (resp. $J$ ) as a leaf.

Claim. If $\log |\alpha| / \log |\beta|$ is irrational then the points of tangency of $f^{-k}(J)$ with $\mathscr{F}_{p}^{u}$ accumulate at $I \cap W^{s}(p)$.

The theorem then follows easily from this claim. In fact, if the claim is correct then by a $C^{\infty}$ small perturbation of $f$ with support in a small neighbourhood of $I \cap W^{s}(p)$ 


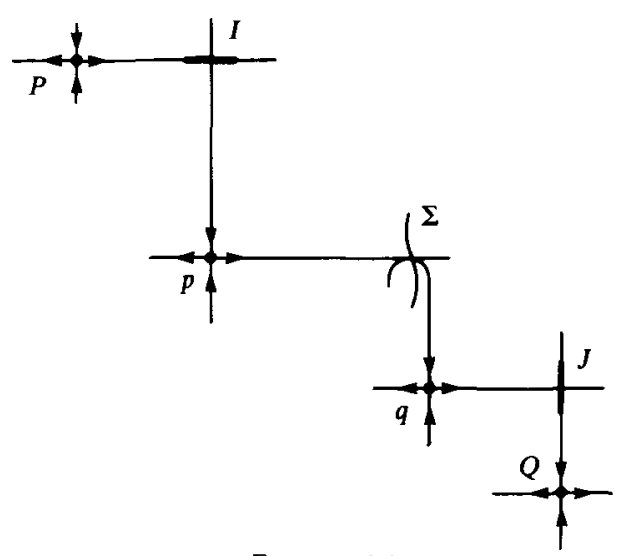

FIGURE 2.2

we can create an arbitrary number of orbits of tangency between $W^{u}(P)$ and $W^{s}(Q)$. Since by corollary 1 each orbit of tangency gives rise to a conjugacy invariant we have that $f$ has infinite modality. So it remains to prove the claim. Since $x$ is a point of quasi-transversal intersection of $W^{u}(p)$ with $W^{s}(q)$ and the tangent line fields to the foliations $\mathscr{F}_{p}^{u}$ and $\mathscr{F}_{q}^{s}$ are $C^{1}$ it follows, from the implicit function theorem, that in a neighbourhood of $x, \mathscr{F}_{p}^{u}$ is transversal to $\mathscr{F}_{q}^{s}$ except along a $C^{1}$ curve $\Sigma$ which is transversal to $W^{u}(p)$ at $x$ (see [Me2]). Let

$$
a_{n}=f^{n}(I) \cap \Sigma \text { and } b_{n}=f^{-n}(J) \cap \Sigma \text {. }
$$

If $d$ is a $C^{0}$ metric on $M$ then we have

$$
\begin{aligned}
& d\left(a_{n}, x\right) \simeq c_{1}|\alpha|^{n}, \\
& d\left(b_{n}, x\right) \simeq c_{2}|\beta|^{-n},
\end{aligned}
$$

where $c_{1}, c_{2}$ are positive constants. This follows from lemma 2.2 , the transversality of $\Sigma$ with $W^{u}(p)$ and the fact that $(*)$ is obviously true for appropriate linearising metrics. Now since $\log |\alpha| / \log |\beta|$ is irrational we can find sequences $n(k), m(k) \rightarrow \infty$ such that $|\beta|^{-n(k)} /|\alpha|^{m(k)} \rightarrow c_{1} / c_{2}$. From (*) therefore follows

and therefore:

$$
\frac{d\left(a_{m(k)}, b_{n(k)}\right)}{|\alpha|^{m(k)}} \rightarrow 0,
$$

$$
d\left(f^{-m(k)}\left(b_{n(k)}\right), f^{-m(k)}\left(a_{m(k)}\right)\right) \rightarrow 0 .
$$

Hence $f^{-m(k)}\left(b_{n(k)}\right)$ converges to $W^{s}(p) \cap I$ as $k \rightarrow \infty$. This proves the claim.

\section{A few techniques for constructing conjugacies}

In this section we will develop a few techniques for constructing conjugacies. In $\S \S 4$ and 5 we will employ these techniques in order to construct conjugacies between two nearby maps in $\mathscr{A}$ which satisfy the relevant moduli conditions.

Let us start with making a general comment. It is well known that every diffeomorphism in $\mathscr{A}$ which satisfies certain transversality conditions on invariant manifolds is structurally stable, see [Rb1]. In order to prove these results one has to construct conjugacies between two nearby diffeomorphisms. Basically there are two ways of doing this. One method is functional analytic. Here one defines an 
operator on an appropriate space of homeomorphisms and shows this operator has a hyperbolic fixed point, see [Rb1]. The other method is a geometric proof using invariant foliations, see [P1], [PS], [Me1], [Me2]. (For Axiom A diffeomorphisms this geometric method has only been developed in dimension two, see [Me1]). In any case it is probably difficult to use the functional analytic methods since all the operators will be non-hyperbolic, see [Rb2]. Therefore we will give geometric constructions using invariant foliations. These methods also enable us to keep track of all the freedom one has in constructing the conjugacies. Let us now define these invariant foliations and explain how they are used.

Take a hyperbolic periodic point $p$ (of saddle type) of a diffeomorphism $f$. An unstable foliation $\mathscr{F}_{p}^{u}$ (tubular family) for $p$ is a continuous retraction $\pi_{u}: V^{u} \rightarrow$ $W^{s}(\mathcal{O}(p))$, where $\mathscr{O}(p)$ is the orbit of $p$ and $V^{u}$ is a neighbourhood of $W^{u}(\mathcal{O}(p))$ with: (i) $\left(\pi_{u}\right)^{-1}(p)=W^{u}(p)$; (ii) the foliation whose leaves are fibers of $\pi_{u}$ is $f$-invariant. Similarly we define the stable foliation $\mathscr{F}_{p}^{s}$ for $p$. Using the $\lambda$-Lemma it is easy to construct invariant unstable foliations, see [P1]. The leaves in fact can be chosen differentiably ( $C^{k}$ if $f$ is $C^{k}$ ) but the field of tangent lines to the leaves is only continuous in general (however see the appendix where we prove this foliation is sometimes more smooth). The use of this foliation is explained by the following lemma whose proof is straightforward.

LEMMA. Let $p, \vec{p}$ be hyperbolic fixed points of $f$ and $\bar{f}$ with unstable foliations $\mathscr{F}_{p}^{u}, \mathscr{F}_{\bar{p}}^{u}$. Let $h$ be a map from a neighbourhood $\mathcal{N}_{p}$ of $W^{u}(p)$ to a neighbourhood of $W^{u}(\bar{p})$ with the following properties: (i) $h f=\bar{f} h$; (ii) $\left(h \mid W^{s}(p)\right): W^{s}(p) \rightarrow W^{s}(\bar{p})$ is continuous; (iii) $h \mid\left(\mathcal{N}_{p}-W^{s}(p)\right)$ is continuous; (iv) $h$ preserves the unstable foliations. Then $h$ is continuous.

In order to construct global conjugacies it is necessary that all unstable foliations fit together nicely. So let $p, q$ be hyperbolic periodic points of $f$ with $W^{u}(p) \cap$ $W^{s}(q) \neq \varnothing$. We say that the unstable foliations $\mathscr{F}_{p}^{u}$ and $\mathscr{F}_{q}^{u}$ are compatible if each leaf of $\mathscr{F}_{p}^{u}$ contains a leaf of $\mathscr{F}_{q}^{u}$. In order to construct a conjugacy $h$ between two nearby Morse-Smale diffeomorphisms one first constructs conjugacies on the stable manifolds of periodic orbits of saddle type preserving the unstable foliations. Here one proceeds inductively using the natural dynamical ordering of periodic orbits starting at sources and ending up at sinks. Then we extend $h$ to fundamental domains of the sinks again preserving these foliations. The conjugacy equation $(h f=\bar{f} h)$ gives a unique extension of $h$ to the whole manifold. From the lemma above it follows that $h$ is continuous. In this approach the compatibility of the foliations is essential.

In our case we have tangencies of invariant manifolds. Therefore some leaves of unstable foliations do not have a unique intersection with stable manifolds. Hence it is impossible to construct a compatible system of unstable foliations. So we will have to use both the unstable and the stable foliations. In the Morse-Smale case these foliations are transversal but here they can be tangent. The geometry of their intersection is extremely complicated (especially when higher order tangencies are involved). So we are forced to make careful adjustments of these foliations in order 
to get a good control of their intersection. These modifications near the tangencies must be globalised in order to keep the foliations invariant. For that we need some careful estimates which we will make in $\S 3(\mathrm{a})$ using a generalised $\lambda$-Lemma proved in the appendix.

There is another difference with the Morse-Smale case. Here we will have to define the conjugacy in a whole neighbourhood of the tangency points first. Then we have the problem of extending $h$ continuously to the closure of the orbit of this neighbourhood. This is done in $\S 3(\mathrm{~b})$.

3(a). Construction of foliations. Let $p$ be a hyperbolic fixed point (of saddle type) of a $C^{\infty}$ diffeomorphism $f$. As usual we say that $D$ is a fundamental domain of $W^{s}(p)$ if each orbit in $W^{s}(p)$ has exactly one intersection with $D$. Similarly $N$ is called a fundamental neighbourhood of $W^{s}(p)$ if each orbit sufficiently near $W^{s}(p)$ has precisely one intersection with $N$. Take a point $x \in W^{u}(p)-\{p\}$ and a small neighbourhood $V_{x}$ of $x$. Let $C_{x, n}$ be a cone-like region which has a tangency of order $n$ with $W^{u}(p)$ at $x$ :

$$
C_{x}=C_{x, n}=\left\{w \in V_{x} ; d\left(w, W^{u}(p)\right) \geq c(d(w, x))^{n}\right\} .
$$

Here $d$ is a $C^{0}$ metric on $M$ and $c$ is a positive constant. Let $\mathscr{F}$ be a $C^{1}$ invariant foliation on $V_{x}$ whose leaf through $x$ is $W^{u}(p)$. For each $a \in V_{x}$ let $L_{a}$ denote the leaf of $\mathscr{F}$ through $a$.

LEMMA 3.1 (Extension of foliations defined on cones). If

$$
\lim _{a \rightarrow x} \frac{\sup \left\{d\left(w, W^{u}(p)\right) ; w \in L_{a} \cap C_{x}\right\}}{\inf \left\{d\left(w, W^{u}(p)\right) ; w \in L_{a} \cap C_{x}\right\}}=1
$$

then there exists an invariant unstable $C^{0}$ foliation $\mathscr{F}_{p}^{u}$ whose restriction to $C_{x, n}$ coincides with $\mathscr{F}$.

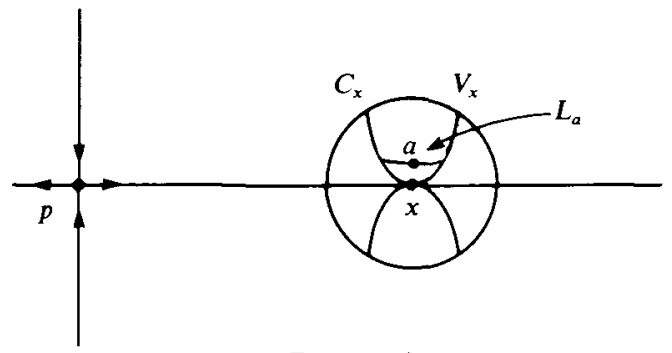

FIGURE 3.1

Remark. If $d$ is a linearizing metric then lemma 3.1 remains true if we take for $C_{x}$ a whole neighbourhood of $x$, provided $V_{x}$ is contained in a fundamental domain. Proof. Take any invariant unstable foliation $\hat{\mathscr{F}}_{p}^{u}$ of $p$. We will modify $\hat{\mathscr{F}}_{p}^{u}$ so that restricted to $C_{x}$ it will coincide with $\mathscr{F}$. Let $N_{x}$ be the connected component containing $x$ of a fundamental neighbourhood of $W^{u}(p)$ such that $N_{x}-C_{x}$ consists of two components as in figure 3.2. For $N_{x}$ sufficiently small $\hat{\mathscr{F}}_{p}^{u} \cap N_{x} \cap \partial C_{x}$ has precisely two intersections.

Define the new foliation $\mathscr{F}_{p}^{u}$ in $N_{x}$ by taking this foliation identical to $\hat{\mathscr{F}}_{p}^{u}$ on $N_{x}-C_{x}$ and identical to $\mathscr{F}$ on $C_{x}$. Clearly $\mathscr{F}_{p}^{u}$ extends uniquely into an invariant foliation 


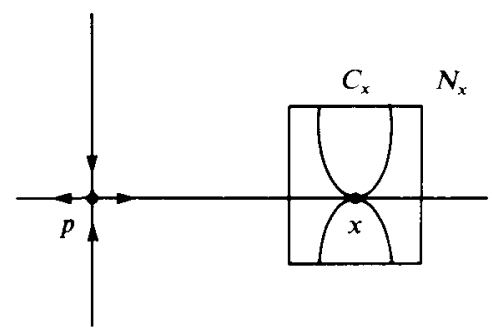

FIGURE 3.2

on a neighbourhood of $W^{u}(p)-\{p\}$. We must show that the closure of each leaf of $\mathscr{F}_{p}^{u}$ has exactly one intersection with $W^{s}(p)$. More precisely, we must check that there is a continuous projection $\pi_{p}$ on $W^{s}(p)$ along the leaves of $\mathscr{F}_{p}^{u}$. In order to show this it suffices to prove that for any sequence $a(i) \in N_{x}$ such that $f^{-i}(a(i)) \rightarrow y \in$ $W^{s}(p)$ as $i \rightarrow \infty$ one has $f^{-i}\left(L_{a(i)}\right) \rightarrow y$. Here $L_{a(i)}$ denotes the leaf of $\mathscr{F}_{p}^{u} \cap N_{x}$ through $a(i)$. Now if $a(i)$ and $x(i) \in L_{a(i)}$ are both contained in the same component of $N_{x}-C_{x}$ it follows from the fact that $\mathscr{F}_{p}^{u}$ is identical to the unstable foliation $\hat{\mathscr{F}}_{p}^{u}$ (on $N_{x}-C_{x}$ ) that $f^{-i}(x(i)) \rightarrow y$. So it suffices to consider sequences $a(i) \in N_{x} \cap C_{x}$ and show that for $x(i) \in L_{a(i)} \cap C_{x}$ one has $f^{-i}(x(i)) \rightarrow y$. But from equation (3.1) it follows that

$$
d\left(a(i), W^{u}(p)\right) \simeq d\left(x(i), W^{u}(p)\right) .
$$

From equation (3.2) and lemma 2.3 it follows that $f^{-i}(a(i)) \rightarrow y$ if and only if $f^{-i}(x(i)) \rightarrow y$. This finishes the proof of the lemma.

It will be convenient to give a general way of constructing foliations $\mathscr{F}$ which satisfy equation (3.1), in terms of some $C^{1}$-coordinate systems near $x$. In fact take a $C^{1}$-coordinate system $(u, v)$ near $x$ so that

$$
\{v=0\}=W^{u}(p), \quad\{(u, v)=(0,0)\}=\{x\} .
$$

In these coordinates the leaves of $\mathscr{F}$ are in the form

$$
L_{a}=\{v=g(u, a)\}
$$

with $g(0, a)=a$. Write $g_{a}(u)=g(u, a)$.

LEMMA 3.2 (Construction of foliations in cases which can be extended to tubular families). Let $n$ be the order of tangency of the cone $C_{x, n}$ with $W^{u}(p)$ and let $g: \mathbb{R}^{2} \rightarrow \mathbb{R}$ be so that:

(1) the $n$-jet $j^{n} g_{a}(u)$ varies $C^{1}$ or, in other words, $a \mapsto j^{n} g_{a}(u)$ is $C^{1}$.

(2) $u \mapsto g_{a}(u)$ is $C^{\infty}$.

(3) $j^{n} g_{0}(u)=0$.

Then the conclusion of lemma 3.1 holds, i.e. the foliation $\mathscr{F}$ on $C_{x, n}$ (corresponding to $g$ as above) can be extended to an unstable foliation.

Proof. According to the theorem of Taylor one has

$$
g(u, a)=a+\varphi_{2}(a) \cdot u+\cdots+\varphi_{n}(a) \cdot u^{n}+R(u, a) \cdot u^{n},
$$

with $R(u, a) \rightarrow 0$ as $(u, a) \rightarrow(0,0)$, and with $\varphi_{i} C^{1}$ so that $\varphi_{i}(0)=0$. Now for $(i, v) \in$ 
$C_{x, n}$ one has for some constant $c_{1}$,

$$
|u|^{n} \leq c_{1} \cdot|v| .
$$

Now let $(u, v)=(u, g(u, a))$. Since $\varphi_{i}$ is $C^{\prime}$ and $\varphi_{i}(0)=0$ one has from (3.3) and (3.4) that:

$$
|v|=|g(u, a)| \leq c_{2} \cdot|a|+c_{1} \cdot|v| \cdot|R(u, a)|
$$

for some constant $c_{2}$. From this it follows that

$$
|v| \leq c_{3}|a| \text { and }|u|^{n} \leq c_{4} \cdot|a|
$$

for some constants $c_{3}$ and $c_{4}$. Therefore

$$
\begin{aligned}
\left|\frac{v}{a}-1\right| & =\left|\frac{g(u, a)}{a}-1\right| \\
& \leq\left|\frac{\varphi_{2}(a)}{a}\right| \cdot|u|+\cdots+\left|\frac{\varphi_{n}(a)}{a}\right| \cdot|u|^{n}+\left|\frac{u^{n}}{a}\right| \cdot|R(u, a)| \\
& \leq c_{5} \cdot|u|+c_{4} \cdot|R(u, a)| .
\end{aligned}
$$

From this it follows that

$$
\left|\frac{v}{a}-1\right|=\left|\frac{g(u, a)}{a}-1\right| \rightarrow 0
$$

as $(x, a) \rightarrow(0,0)$. Obviously equation (3.6) implies equation (3.1) in lemma 3.1.

We will use lemma 3.2 in order to modify foliations near certain parts of the manifold. Let $f$ be a diffeomorphism with a non-transversal saddle-connection as in figures (3.3) and (3.4).

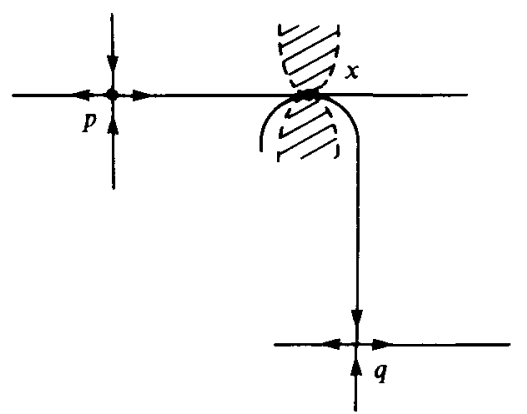

FIGURE 3.3. Tangency of even order

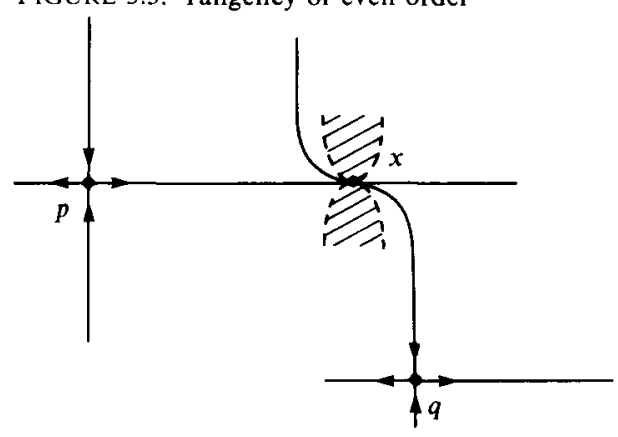

Figure 3.4. Tangency of odd order 
Assume that $W^{u}(p)$ and $W^{s}(q)$ have a tangency of order $n$ at $x$. Let $\mathscr{F}_{p}^{u}$ and $\mathscr{F}_{q}^{s}$ be respectively the unstable foliation at $p$ and the stable foliation at $q$. According to the theorem in the appendix one can choose these foliations $C^{1}$ so that the fields of tangent lines are $C^{1}$ and so that the leaves of these foliations are $C^{\infty}$. We first show that $\mathscr{F}_{p}^{u}$ and $\mathscr{F}_{q}^{s}$ can only be tangent in a certain cone $C_{x, n}$.

LEMMA 3.3. Let $\mathscr{F}, \mathscr{F}^{\prime}$ be $C^{1}$ foliations on a neighbourhood of the origin of $\mathbb{R}^{2}$ such that the leaves are $C^{r+1}, r \geq 1$, the fields of tangent lines are $C^{1}$ and the leaf $\mathscr{F}^{\prime}(0)$ of $\mathscr{F}^{\prime}$ through 0 has contact $r+1$ with $\mathscr{F}(0)$. Let $T$ be the set of points where $\mathscr{F}$ is tangent to $\mathscr{F}^{\prime}$. Then the contact of $T$ with $\mathscr{F}(0)$ at 0 is less than $r+1$, that is more precisely, either $T-\{0\}=\varnothing$ or

where $d$ is some $C^{0}$ metric.

$$
\lim _{\substack{z \in T-\{0\} \\ z \rightarrow 0}} \frac{d(z, \mathscr{F}(0))}{(d(z, 0))^{r+1}}=\infty,
$$

Proof. Choose a $C^{r+1}$ coordinate system $(u, v)$ in a neighbourhood of 0 such that $\mathscr{F}(0,0)=\{(u, v) \mid v=0\}$ and $\mathscr{F}^{\prime}(0,0)=\left\{(u, v) \mid v=u^{r+1}\right\}$. Denote by $\varphi(u, v)$ the tangent of the angle of $\mathscr{F}^{\prime}(u, v)$ with the horizontal line through $(u, v)$ and by $\psi(u, v)$ the tangent of the angle between $\mathscr{F}(u, v)$ and $\mathscr{F F}^{\prime}(u, v)$. Hence $\varphi$ and $\psi$ are $C^{1}$, $\psi(u, 0)=\varphi(u, 0), \varphi\left(u, u^{r+1}\right)=(r+1) \cdot u^{r}$ and $T=\{(u, v) \mid \psi(u, v)=0\}$. By the mean value theorem we have

and

$$
\psi(u, v)=\psi(u, 0)+\frac{\partial \psi}{\partial v}(u, \bar{v}) \cdot v
$$

$$
\psi(u, 0)=\varphi(u, 0)=\varphi\left(u, u^{r+1}\right)-\frac{\partial \varphi}{\partial v}(u, \tilde{v}) \cdot u^{r+1},
$$

for some $\bar{v}$ between 0 and $v$ and some $\tilde{v}$ between 0 and $u^{r+1}$. Thus

and

$$
\psi(u, v)=(r+1) \cdot u^{r}-\frac{\partial \varphi}{\partial v}(u, \tilde{v}) \cdot u^{r+1}+\frac{\partial \psi}{\partial v}(u, \bar{v}) \cdot v
$$

$$
\frac{\psi(u, v)}{u^{r+1}}=\frac{r+1}{u}-\frac{\partial \varphi}{\partial v}(u, \tilde{v})+\frac{\partial \psi}{\partial v}(u, \bar{v}) \cdot \frac{v}{u^{r+1}}
$$

If $(u, v) \in T$ we have $\psi(u, v)=0$ and since $\partial \psi / \partial v$ and $\partial \varphi / \partial v$ are bounded, we have

This clearly proves the lemma.

$$
\lim _{\substack{(u, v) \in T \\ u \rightarrow 0}} \frac{|v|}{|u|^{r+1}}=+\infty .
$$

Now we will put lemma 3.2 and lemma 3.3 together and show how to modify the foliations $\mathscr{F}_{p}^{u}$ and $\mathscr{F}_{q}^{s}$ so that they are tangent only along a curve.

Consider any $C^{0}$ metric $d$. Since $W^{u}(p)$ and $W^{s}(q)$ have a tangency of order $n$ one has (as in lemma (2.6)) that

$$
\lim _{\substack{w \in W^{s}(q) \\ w \rightarrow x}} \frac{d\left(w, W^{u}(p)\right)}{\left(d(w, x)^{n}\right.}=Q_{x}
$$

for some positive number $Q_{x}$. Now let $T$ be the set where $\mathscr{F}_{p}^{u}$ and $\mathscr{F}_{q}^{s}$ are tangent, and let as before

$$
C_{x, n}=\left\{w \mid d\left(w, W^{u}(p)\right) \geq c \cdot(d(w, x))^{n}\right\} .
$$


According to lemma 3.3, for any $c>0$ there is a neighbourhood $V_{x}$ of $x$ so that

$$
T \cap V_{x} \subset C_{x, n} \text {. }
$$

So take $c>Q_{x}$, and $V_{x}$ so small that $C_{x, n} \cap W^{s}(q)=\{x\}=C_{x, n} \cap W^{u}(p)$. In other words, outside the set $C_{x, n}$ the foliations $\mathscr{F}_{p}^{u}$ and $\mathscr{F}_{q}^{s}$ have no tangencies (in $V_{x}$ ), so we will modify these foliations in $C_{x, n}$. We can modify either $\mathscr{F}_{p}^{u}$ or $\mathscr{F}_{q}^{s}$ according to whichever is more convenient. Let us change $\mathscr{F}_{q}^{s}$. In order to do this take $C^{1}$-coordinates $(u, v)$ near $x$ so that $\{v=0\}=W^{u}(p)$, and so that the leaves of $\mathscr{F}_{p}^{u}$ are of the form $\{v=c\}$, i.e. parallel to the $\{v=0\}$ line. From the appendix it follows that this is possible. Clearly we can write $W^{s}(q)$ in the form $W^{s}(q)=\{v=q(u)\}$ where $q$ is some $C^{1}$ function and $q(0)=0$. From the remark above lemma 2.3 it follows that $q(u) / u^{n}$ converges to a number $Q \neq 0$ as $u \rightarrow 0$. We will treat the case that $n$ is even and the case that $n$ is odd separately.

Case 1: $n$ is even. Consider the foliation $\{v=g(u, a)\}, a \in \mathbb{R}$, where $g(u, a)=Q u^{n}+a$. According to lemma 3.2 we can change the foliation $\mathscr{F}_{q}^{s}$ so that inside $C_{x, n}$ the leaves of this foliation are of the form $\{v=g(u, a)\}$. From this and lemma 3.3 it follows that then $\mathscr{F}_{p}^{u}$ and $\mathscr{F}_{q}^{s}$ are tangent exactly along the line $\{\boldsymbol{u}=0\}$.

Case 2: $n$ is odd. In this case change the foliation $\mathscr{F}_{q}^{s}$ so that inside $C_{x, n}$ the leaves of this foliation are of the form $\{v=g(u, a)\}$, where $g(u, a)=Q u^{n}+a^{2} \cdot u+a$. From this it follows that then $\mathscr{F}_{p}^{u}$ and $\mathscr{F}_{q}^{s}$ are tangent only at the point $x$.

Remark. The set $C_{x, n} \backslash\{x\}$ consists of two components. Sometimes it will be necessary to modify $\mathscr{F}_{p}^{u}$ in one component and $\mathscr{F}_{q}^{s}$ in the other component.

3(b). Construction of conjugacies. Now we give a lemma which will show us how to use these foliations in the construction of conjugacies. As before let $V_{x}$ be a neighbourhood of a point $x \in W^{u}(p)-\{p\}$ and let,

$$
C_{x, n}=\left\{w \in V_{x} ; d\left(w, W^{u}(p)\right) \geq c \cdot\{d(w, x)\}^{n}\right\} .
$$

(Here $d$ is a $C^{0}$ metric as before).

We consider the following situation. Let $p, \bar{p}$ be hyperbolic fixed points of $C^{2}$ diffeomorphisms $f, \bar{f}$. Let the contracting eigenvalue of $f$ (resp. $\bar{f}$ ) be $\alpha$ (resp. $\bar{\alpha}$ ), and let

$$
\delta=\frac{\log |\bar{\alpha}|}{\log |\alpha|}, \quad \text { i.e. } \quad|\alpha|^{\delta}=|\bar{\alpha}| .
$$

Let $\mathscr{F}_{p}^{u}$ (resp. $\mathscr{F}_{\bar{p}}^{u}$ ) be the unstable foliation for $f$ (resp. $\bar{f}$ ). Let $d, \bar{d}$ be two $C^{0}$ metrics. We have the following.

LEMMA 3.4. Let $h: V_{x} \rightarrow V_{\bar{x}}$ be a homeomorphism satisfying the properties:

(i) $\lim _{\substack{w \in C_{x, n} \\ w \rightarrow x}} \frac{\bar{d}\left(h(w), W^{u}(\bar{p})\right)}{\left(d\left(w, W^{u}(p)\right)^{\delta}\right.}$ exists and is positive.

(ii) The restriction of $h$ to each connected component of $V_{x}-C_{x}$ maps leaves of the restriction of the foliation $\mathscr{F}_{p}^{u}$ to this connected component into leaves of $\mathscr{F}_{\bar{p}}^{u}$.

Then hextends to a homeomorphism of a neighbourhood of $p$, conjugating $f$ and $\bar{f}$. 
Proof. Let $d^{\prime}$ and $\bar{d}^{\prime}$ be metrics induced by $C^{1}$-linearising coordinates for $f$ (resp. $\bar{f}$ ). From lemma 2.2 in $\S 2$ it follows that

$$
\lim _{\substack{w \rightarrow x \\ w \in C_{x, n}}} \frac{\bar{d}^{\prime}\left(h(w), W^{u}(\bar{p})\right)}{\left\{d^{\prime}\left(w, W^{u}(p)\right\}^{\delta}\right.}=l \neq 0 .
$$

Furthermore since $d^{\prime}$ and $\bar{d}^{\prime}$ are induced by linearising coordinates one has for sequences $x_{i} \rightarrow x$ that $f^{-n(i)}\left(x_{i}\right)$ converges to some point $z \in W^{s}(p)$ if and only if

$$
d^{\prime}\left(x_{i}, W^{u}(p)\right) \simeq d^{\prime}(p, z) \cdot|\alpha|^{n(i)},
$$

and a similar statement holds for $\bar{f}$. From this, equation (3.6), and the fact that $|\alpha|^{\delta}=|\bar{\alpha}|$, it follows that $h$ extends to a homeomorphism of the closure of $\bigcup_{k=0}^{\infty} f^{-k}\left(C_{x, n}\right)$ conjugating $f$ and $\bar{f}$.

Now assume $V_{x}$ is sufficiently small so that $f\left(V_{x}\right) \cap V_{x}=\varnothing$. We can extend $h$ to $V_{x}$ by taking any extension of $h \mid C_{x, n}$ which maps leaves of $\mathscr{F}_{p}^{u}$ to leaves of $\mathscr{F}_{\bar{p}}^{u}$. It follows that $h$ extends to the closure of $\bigcup_{k=0}^{\infty} f^{-k}\left(V_{x}\right)$.

Let us now extend $h$ to a neighbourhood of $W^{u}(p)$. Take a leaf $F$ of $\mathscr{F}_{p}^{u}$. For simplicity we can assume that $V_{x}$ is chosen so that if $F$ intersects $V_{x}$, then $F \backslash\left(V_{x} \cup\right.$ $f^{-1}\left(V_{x}\right)$ ) consists of three components, see figure 3.5. Consider the component of $F \backslash\left(V_{x} \cup f^{-1}\left(V_{x}\right)\right)$ with boundary points $x_{1} \in V_{x}$ and $x_{2} \in f^{-1}\left(V_{x}\right)$.

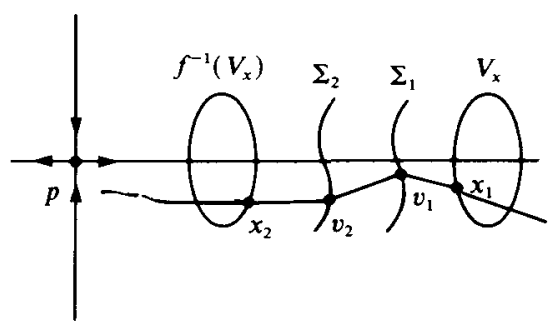

FiguRE 3.5

Consider $h\left(x_{1}\right)$ and $h\left(x_{2}\right)$. In general $h\left(x_{1}\right)$ and $h\left(x_{2}\right)$ need not lie on one leaf of $\mathscr{F}_{\bar{p}}^{u}$. Therefore we will modify $\mathscr{F}_{\bar{p}}^{u}$. In fact take two curves $\bar{\Sigma}_{1}, \bar{\Sigma}_{2}$ transversal to $W^{u}(\bar{p})$ between $h\left(V_{x}\right)$ and $\bar{f}^{-1}\left(h\left(V_{x}\right)\right)$ as in figure 3.5. The leaf $\bar{F}_{i}$ of $\mathscr{F}_{\bar{p}}^{u}$ through' $h\left(x_{i}\right)$ has an intersection $v_{i}$ with $\bar{\Sigma}_{i}$. Take a leaf $L_{v_{1}}$ in the strip between $\Sigma_{1}$ and $\Sigma_{2}$ which is piecewise linear with respect to the linearising coordinates for $\bar{f}$ such that $L_{v_{1}} \cap \bar{\Sigma}_{1}=v_{1}, L_{v_{1}} \cap \bar{\Sigma}_{2}=v_{2}$. Do this for every $v_{1} \in \bar{\Sigma}_{1}$. Now define a new foliation ' $\mathscr{F}_{\bar{p}}^{u}$ ' which is identical to $\mathscr{F}_{\bar{p}}^{u}$ outside the strip between $\Sigma_{1}$ and $\Sigma_{2}$ and identical to the piecewise linear one inside this strip. It is easy to see that ' $\mathscr{F}_{\bar{p}}^{u}$ ' is an honest invariant unstable foliation for $\bar{p}$, using lemma 3.1 .

Now define a conjugacy $h$ on a neighbourhood of $W^{u}(p)$ by taking any extension of $h \mid V_{x}$ which maps leaves of $\mathscr{F}_{p}^{u}$ onto leaves of ' $\mathscr{F}_{\bar{p}}^{u}$ '. From the construction above it follows that this is possible.

Now we consider the situation that a homeomorphism $h$ is only defined on a cone-like set $C_{x}$. More specifically consider the situation that $f$ has hyperbolic saddle-points $p, q$ such that $W^{u}(p)$ and $W^{s}(q)$, have a tangency of (even) order $n$ at a point $x$. 


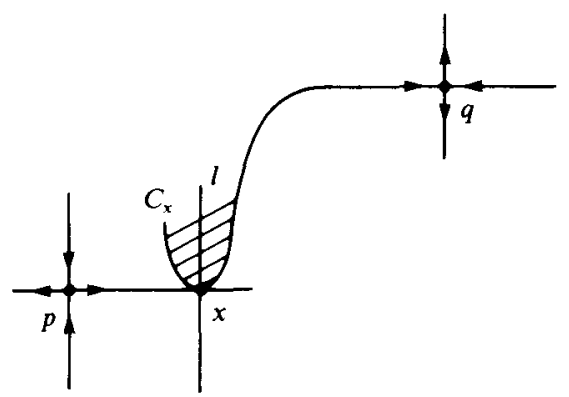

FIGURE 3.6

Let $C_{x}$ be the cone-like set as in figure 3.6, and let $\bar{f}$ be a diffeomorphism so that $W^{u}(\bar{p})$ and $W^{s}(\bar{q})$ also have a tangency of order $n$ at $\bar{x}$. Assume $h: C_{x} \rightarrow \bar{C}_{x}$ is a homeomorphism. When can we extend $h$ to a conjugacy on a neighbourhood of $p$ ? In order to investigate this question take a $C^{0}$-metric $d$ near $x$ and define

$$
Q_{x}=\lim _{\substack{w \in W^{s}(q) \\ w \rightarrow x}} \frac{d\left(w, W^{u}(p)\right)}{\{d(w, x)\}^{n}} .
$$

As we have shown before, $Q_{x}$ is well defined and $Q_{x} \neq 0$. Define $Q_{\bar{x}}$ similarly. Let $l$ be a $C^{\infty}$ curve through $x$ transversal to $W^{\text {u }}(p)$ and take a similar curve $\bar{l}$ for $\bar{f}$. We assume that $h(l)=\bar{l}$ and that the following limits exist and are positive:

$$
C_{1}=\lim _{\substack{w \rightarrow x \\ w \in C_{x}-l}} \frac{\bar{d}(h(w), \bar{l})}{d(w, l)} \quad \text { and } \quad C_{2}=\lim _{\substack{w \rightarrow x \\ w \in C_{x}-W^{s}(q)}} \frac{\bar{d}\left(h(w), W^{s}(\bar{q})\right)}{d\left(w, W^{s}(q)\right)} .
$$

Also assume the contracting eigenvalue of $f$ at $p$ is equal to the contracting eigenvalue of $\bar{f}$ at $\bar{p}$. The numbers $Q_{\bar{x}}, C_{1}, C_{2}$ do depend on the choice made for the metrics $d, \bar{d}$. However we have the following result.

LeMMA 3.5. Let $h$ be a homeomorphism as above. Then:

(1) the equality $Q_{x}=\left(C_{1}^{n} / C_{2}\right) Q_{\bar{x}}$ does not depend on the metric d, $\bar{d}$; (i.e. if the equality holds for some $C^{0}$ metrics $d, \bar{d}$ then it holds for all $C^{0}$ metrics);

(2) if $Q_{x}=\left(C_{1}^{n} / C_{2}\right) Q_{\bar{x}}$ then the homeomorphism $h$ can be extended to a conjugacy on a neighbourhood of $p$.

Proof. (1) Let $d^{\prime}$ be another $C^{0}$ metric instead of $d$. From lemma 2.2 and the remark below this lemma we have that there exists $\lambda>0$ so that

$$
\lim _{\substack{w \rightarrow x \\ w \in W^{s}(q)}} \frac{d\left(w, W^{u}(p)\right)}{d^{\prime}\left(w, W^{u}(p)\right)}=\lambda=\lim _{\substack{w \rightarrow x \\ w \in C_{x}-W^{s}(p)}} \frac{d\left(w, W^{s}(q)\right)}{d^{\prime}\left(w, W^{s}(q)\right)} .
$$

Furthermore if $w \in W^{s}(q)$ converges to $x$ then from lemma 2.1 it follows that $d(w, x) / d^{\prime}(w, x)$ converges to a positive constant $\mu$. On the other hand one has $d(w, l) \simeq d(w, x), d^{\prime}(w, l) \simeq d^{\prime}(w, x)$ for $w \in W^{s}(q)$ converging to $x$ since $W^{s}(q)$ and $W^{u}(p)$ are tangent to each other and transversal to $l$. Hence for some $\mu>0$

$$
\lim _{\substack{w \in W^{s}(q) \\ w \rightarrow x}} \frac{d(w, x)}{d^{\prime}(w, x)}=\mu=\lim _{\substack{w \in W^{s}(q) \\ w \rightarrow x}} \frac{d(w, l)}{d^{\prime}(w, l)}
$$


It follows immediately that the equality $Q_{x}=\left(C_{1}^{n} / C_{2}\right) Q_{\bar{x}}$ does not depend on the choice of $d$. Similarly it does not depend on the choice of $\bar{d}$.

(2) Take $C^{\infty}$ coordinates $(u, v)$ at $x$ so that $W^{u}(p)=\{v=0\}, W^{s}(q)=\left\{v=Q_{x} u^{n}\right\}$ and $l=\{u=0\}$. From (1) we can take for $d$ the usual Euclidean metric in this coordinate system. Take $w \in C_{x}$ and let $w$ have coordinates $(u, v)$. Then

$$
d\left(w, W^{u}(p)\right)=v=Q_{x}(d(w, l))^{n}+v-Q_{x}(d(w, l))^{n} .
$$

Furthermore

$$
v-Q_{x}(d(w, l))^{n} \simeq d\left(w, W^{s}(q)\right)
$$

as $w \rightarrow x$ (see the arguments used in the remark below lemma 2.2). Now we use the following elementary fact. Take sequences $a_{i}, b_{i}, c_{i}, d_{i}$ of positive numbers such that $a_{i} \simeq b_{i}+c_{i}$ and $c_{i} / d_{i}$ converges to 1 . Then $a_{i} \simeq b_{i}+d_{i}$. It follows that

$$
d\left(w, W^{u}(p)\right)=Q_{x}(d(w, l))^{n}+d\left(w, W^{s}(q)\right)
$$

and similarly

$$
\bar{d}\left(h(w), W^{u}(\bar{p})\right) \simeq Q_{\bar{x}}(\bar{d}(h(w), \bar{l}))^{n}+\bar{d}\left(h(w), W^{s}(\bar{q})\right) .
$$

Using a similar elementary computation as above one concludes that

$$
\frac{\bar{d}\left(h(w), W^{u}(\bar{p})\right)}{d\left(w, W^{u}(p)\right)}
$$

converges for $w \in C_{x}$ converging to $x$ if and only if

$$
Q_{x}=\frac{C_{1}^{n}}{C_{2}} Q_{\bar{x}}
$$

(In this case the limit is $C_{2}$ ). Using lemma 3.4 it follows that we can extend $h$ to a conjugacy near $p$.

4. Construction of conjugacies when all invariant manifolds except $W^{u}(p)$ and $W^{s}(q)$ intersect each other transversally

Suppose that $p$ and $q$ are hyperbolic saddle-points and that $W^{u}(p)$ and $W^{s}(q)$ have $k$-orbits of non-transversal intersection.

In order to state the next theorem we need some notation. Let $\alpha$ (resp. $\beta$ ) be the contracting (resp. expanding) eigenvalue of $f$ at $p$ (resp. $q$ ). Choose a fundamental domain $D_{p}^{u}$ in $W^{u}(p)$ and let $z_{1}, \ldots, z_{k}$ be the points in $D_{p}^{u}$ where $W^{u}(p)$ and $W^{s}(q)$ are tangent. Order these points so that $z_{i}$ is between $p$ and $z_{i+1}$ on the curve $W^{u}(p)$. Then take a $C^{1}$ curve $\Sigma_{i}$ at $z_{i}$ transversal to $W^{u}(p)$ and $W^{s}(q)$ and take linearising metrics $d_{p}, d_{q}$ at $p$ and $q$ respectively. Define

$$
\Delta_{z_{i}}=\lim _{\substack{w \rightarrow z_{i} \\ w \in \Sigma_{i}}} \frac{d_{p}\left(w, z_{i}\right)}{d_{q}\left(w, z_{i}\right)}
$$

Let $p_{1}, \ldots, p_{n(p)}\left(\operatorname{resp} . q_{1}, \ldots, q_{n(q)}\right)$ be the saddles whose unstable (resp. stable) manifolds intersect $W^{s}(p)$ (resp. $\left.W^{u}(q)\right)$. Denote the contracting (resp. expanding) eigenvalue of $f$ at $p_{i}\left(\operatorname{resp} q_{i}\right)$ by $A_{i}$ (resp. $\left.B_{i}\right)$, and the linearising metrics by respectively $d_{p_{i}}$ and $d_{q_{i}}$. Choose fundamental domains $D_{p}^{s}$ in $W^{s}(p)$ and $D_{q}^{u}$ in $W^{u}(q)$. Let $x_{1}, \ldots, x_{l(p)}\left(\right.$ resp. $\left.y_{1}, \ldots, y_{l(q)}\right)$ be the intersections of the unstable (resp. stable) 
manifolds of the saddles $p_{i}$ 's (resp. $q_{i}$ 's) with $D_{p}^{s}$ (resp. $D_{q}^{u}$ ) ordered as above. Let $I=\left\{i \mid W^{u}(p)\right.$ and $W^{s}(q)$ have a tangency of even order at $\left.z_{i}\right\}$,

$$
I_{p_{i}}=\left\{j ; x_{j} \in W^{u}\left(p_{i}\right) \cap D_{p}^{s}\right\}
$$

and

Define

$$
I_{q_{i}}=\left\{j ; y_{j} \in W^{s}\left(q_{i}\right) \cap D_{q}^{\mu}\right\}
$$

$$
\begin{array}{ll}
\Delta_{p_{i}, x_{j}}=\lim _{\substack{w \rightarrow x_{j} \\
w \in W^{s}(p)}} \frac{d_{p_{i}}\left(w, x_{j}\right)}{d_{p}\left(w, x_{j}\right)} & \text { if } x_{j} \in W^{u}\left(p_{i}\right) \cap D_{p}^{s} \\
\Delta_{q_{i}, y_{j}}=\lim _{\substack{w \rightarrow y_{j} \\
w \in W^{u}(q)}} \frac{d_{q_{i}}\left(w, y_{j}\right)}{d_{q}\left(w, y_{j}\right)} & \text { if } y_{j} \in W^{s}\left(q_{i}\right) \cap D_{q}^{u} .
\end{array}
$$

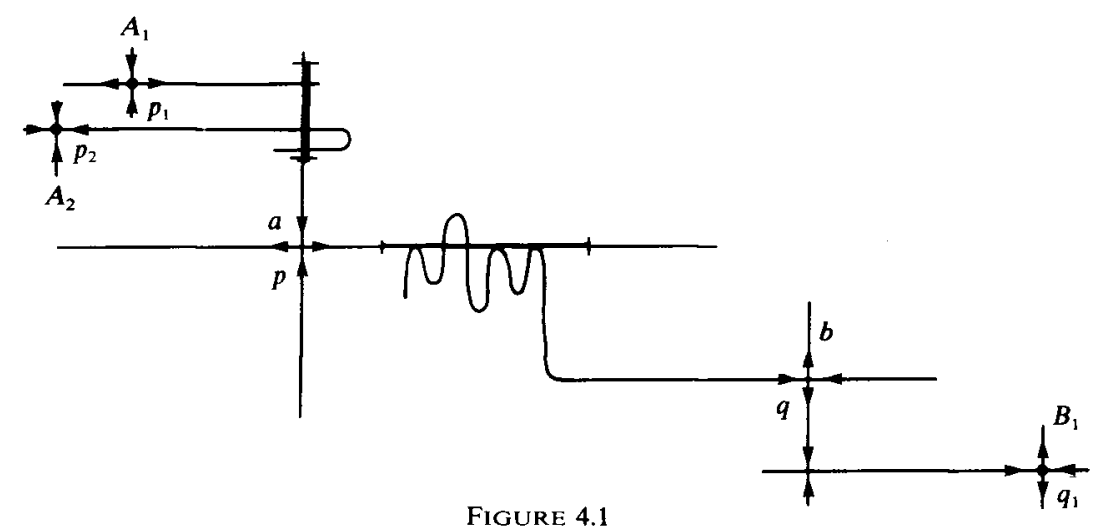

Let $f, \bar{f} \in \mathscr{M}$ be as above and $C^{1}$ near each other. Assume that all invariant manifolds (except $W^{u}(p)$ and $W^{s}(q)$ ) are transversal to each other. Furthermore suppose that $\bar{f}$ has points $\bar{z}_{i}, \bar{x}_{i}, \bar{y}_{i}, \bar{p}_{i}, \bar{q}_{i}$ corresponding to $z_{i}, x_{i}, y_{i}, p_{i}, q_{i}$, which are ordered in the same way, such that $W^{u}(p), W^{s}(q)$ have a tangency of even order at $z_{i}$ if and only if $W^{u}(\bar{p}), W^{s}(\bar{q})$ have a tangency of even order at $\bar{z}_{i}$.

THEOREM 4.1. The diffeomorphisms $f, \bar{f} \in \mathcal{M}$ as above are conjugate if conditions (M1)-(M5) hold. Here (Mi) is given by:

$$
\frac{\log |\alpha|}{\log |\beta|}=\frac{\log |\bar{\alpha}|}{\log |\bar{\beta}|},
$$

i.e. there exists $\delta>0$ such that $|\alpha|^{\delta}=|\bar{a}|$ and $|\beta|^{\delta}=|\bar{\beta}|$;

$$
\begin{gathered}
\frac{\left(\Delta_{z_{i}}\right)^{\delta}}{\Delta_{\bar{z}_{i}}}=\frac{\left(\Delta_{z_{i}}\right)^{\delta}}{\Delta_{\bar{z}_{j}}} \quad \text { for all } i, j \in I ; \\
A_{i}=\bar{A}_{i} \quad i=1, \ldots, n(p), \\
B_{j}=\bar{B}_{j} \quad j=1, \ldots, n(q) ; \\
\frac{d_{p}\left(x_{i}, p\right)}{d_{p}\left(x_{j}, p\right)}=\left[\frac{d_{\bar{p}}\left(\bar{x}_{i}, \bar{p}\right)}{d_{\bar{p}}\left(\bar{x}_{j}, \bar{p}\right)}\right]^{\delta} \quad i=1, \ldots, l(p), \\
\frac{d_{q}\left(y_{i}, q\right)}{d_{q}\left(y_{j}, q\right)}=\left[\frac{d_{\bar{q}}\left(\bar{y}_{i}, \bar{q}\right)}{d_{\bar{q}}\left(y_{j}, \bar{q}\right)}\right]^{\delta} \quad j=1, \ldots, l(q) ;
\end{gathered}
$$


(M5)

$$
\begin{array}{ll}
\frac{\Delta_{\bar{p}_{i}, \bar{x}_{j}}}{\left|\Delta_{p_{i}, x_{j}}\right|^{\delta-1}} \cdot\left[d_{p}\left(x_{j}, p\right)\right]^{\delta-1}=\frac{\Delta_{\tilde{p}_{i}, \bar{x}_{k}}}{\left|\Delta_{p_{i}, x_{k}}\right|^{\delta-1}} \cdot\left[d_{p}\left(x_{k}, p\right)\right]^{\delta-1} & \text { for all } j, k \in I_{p_{i}}, \\
\frac{\Delta_{\bar{q}_{i}, \bar{y}_{j}}}{\left|\Delta_{q_{i}, y_{j}}\right|^{\delta-1}} \cdot\left[d_{q}\left(y_{j}, q\right)\right]^{\delta-1}=\frac{\Delta_{\bar{q}_{i}, y_{k}}}{\left|\Delta_{q_{i}, y_{k}}\right|^{\delta-1}} \cdot\left[d_{q}\left(y_{k}, q\right)\right]^{\delta-1} & \text { for all } j, k \in I_{q_{i}}
\end{array}
$$

If $\log |\alpha| / \log |\beta|$ is irrational these conditions are also necessary for the existence of a conjugacy.

Remark. Only tangencies of even order lead to conjugacy invariants. However the presence of tangencies of odd order causes the modality to increase because they generate, by perturbations of the diffeomorphism, new tangencies of even order.

Proof. The necessity of the moduli conditions follows from lemma 2.5 and its corollaries. In order to show the sufficiency of these conditions we have to construct conjugacies. We have subdivided the proof in a few cases.

Case 1. Suppose that there is no periodic saddle point $P$ with $W^{u}(P) \cap W^{s}(p) \neq \varnothing$ and similarly no periodic saddle-point $Q$ with $W^{u}(q) \cap W^{s}(Q) \neq \varnothing$. As before let $W^{u}(p)$ and $W^{s}(q)$ be tangent at $k$ orbits $\mathcal{O}\left(z_{1}\right), \ldots, \mathcal{O}\left(z_{k}\right)$. It will turn out that the number of conjugacy invariants is equal to the cardinality of $I$. In particular if $\# I=0$ then there are no conjugacy invariants. Let us now construct a conjugacy between $f$ and $\bar{f}$. First we define $h: W^{s}(p) \rightarrow W^{s}(\bar{p})$ so that

$$
d_{\bar{p}}(h(v), \bar{p})=c\left[d_{p}(v, p)\right]^{\delta},
$$

where $c$ is a positive constant and

$$
\delta=\frac{\log |\bar{\alpha}|}{\log |\alpha|}
$$

From (4.1), (4.2) and the fact that $d_{p}$ is a linearising metric it follows that $h \circ f=\bar{f} \circ h$ restricted to $W^{s}(p)$.

Case 1(a): All tangencies are of odd order (i.e. $\# I=0$ ). Take an unstable family $\mathscr{F}_{p}^{u}$ for $p$ and a stable family $\mathscr{F}_{q}^{s}$ for $q$. Using the construction at the end of $\S 3(\mathrm{a})$ one can modify $\mathscr{F}_{p}^{u}$ and $\mathscr{F}_{q}^{s}$ so that, near $z_{i}, \mathscr{F}_{p}^{u}$ and $\mathscr{F}_{q}^{s}$ are tangent only in $z_{i}$. Define $h: W^{u}(q) \rightarrow W^{u}(\bar{q})$ to be any homeomorphism so that $h \circ f=\bar{f} \circ h$. The homeomorphisms $h: W^{s}(p) \rightarrow W^{s}(\bar{p})$ and $h: W^{u}(q) \rightarrow W^{u}(\bar{q})$ induce maps on the space of leaves of $\mathscr{F}_{p}^{u}$ and $\mathscr{F}_{q}^{s}$. This defines $h: V_{z_{i}} \rightarrow V_{\bar{z}_{i}}$ uniquely, where $V_{z_{i}}$ is a neighbourhood of $z_{i}$. Now we show how to extend $h$ globally. Take a system of invariant foliations $\mathscr{F}_{\Omega_{i}}^{s}$, $\mathscr{F}_{\Omega_{i}}^{u}$ for all basic sets $\Omega_{i}$ in $\Omega(f)$, so that $\mathscr{F}_{\Omega_{i}}^{s}$ and $\mathscr{F}_{\Omega_{j}}^{s}$ (and similarly $\mathscr{F}_{\Omega_{i}}^{u}$ and $\mathscr{F}_{\Omega_{j}}^{u}$ ) are compatible for all basic sets $\Omega_{i}, \Omega_{j}$ except where $\Omega_{i}=\{p\}$ and $\Omega_{j}=\{q\}$. In [P1], [MP] and [Me2] it is shown how to do this when $\Omega(f)$ is finite. If $\Omega(f)$ is infinite then one has to use the methods of [Me1]. Remark that $f \in \mathcal{M}$ implies that there is no point $x$ which is part of a non-trivial basic set (i.e. which is not a periodic orbit) such that $W^{u}(x)$ or $W^{s}(x)$ has intersection with $W^{s}(p)$ or $W^{u}(q)$. As in [Pa1] and [Me1] we proceed by defining $h: W^{u}\left(\Omega_{i}\right) \rightarrow$ $W^{u}\left(\bar{\Omega}_{i}\right)$ for any basic set $\Omega_{i}$ such that $W^{s}\left(\Omega_{i}\right) \cap W^{u}(p) \neq \varnothing$. This induces a map on the space of leaves of $\mathscr{F}_{\Omega_{i}}^{s}$ and from the transversality of $W^{s}(x), x \in \Omega_{i}$, with $W^{u}(p)$ this gives a map $h: W^{u}(p) \cap V \rightarrow W^{u}(\bar{p}) \cap \bar{V}$, where $V$ is a neighbourhood 
of $W^{u}(p) \cap W^{s}\left(\Omega_{i}\right)$. Extend these maps to a conjugacy $h: W^{u}(p) \rightarrow W^{u}(\bar{p})$. Since $\mathscr{F}_{p}^{u}$ and $\mathscr{F}_{q}^{s}$ intersect transversally, except at $z_{i}$, the conjugacies $h: W^{s}(p) \rightarrow W^{s}(\bar{p})$ and $h: W^{u}(p) \rightarrow W^{u}(\bar{p})$ can be extended to a unique conjugacy on a neighbourhood of $p$ which respects all invariant foliations. As in [P1], [MP], [Me1] and [Me2] one extends $h$ globally.

Case 1(b): One even tangency, i.e. \#I=1. Let $z=z_{i}$ be a tangency of $W^{u}(p)$ and $W^{s}(q)$ of even order. Using the construction of the end of $\S 3(a)$ one can modify $\mathscr{F}_{p}^{u}$ and $\mathscr{F}_{q}^{s}$ so that, near $z, \mathscr{F}_{p}^{u}$ and $\mathscr{F}_{q}^{s}$ are tangent along a $C^{1}$-curve $\Sigma$. The homeomorphism $h: W^{s}(p) \rightarrow W^{s}(\bar{p})$ induces a map on the fibers of $\mathscr{F}_{p}^{u}$ and $\mathscr{F}_{\bar{p}}^{u}$, and we get a homeomorphism $h: \Sigma \rightarrow \bar{\Sigma}$. From equation (4.1) we get

$$
d_{\bar{p}}(h(v), \bar{z}) \simeq c\left[d_{p}(v, z)\right]^{\delta} \quad \text { for } v \in \Sigma .
$$

Hence

$$
d_{\bar{q}}(h(v), \bar{z}) \simeq c \frac{\left(\Delta_{z}\right)^{\delta}}{\Delta_{\bar{z}}}\left[d_{q}(v, z)\right]^{\delta}
$$

Using the conjugacy equation $h \circ f=\bar{f} \circ h$ we can extend $h$ to $\bigcup_{j \in Z} f^{j}(\Sigma)$. This set accumulates on $W^{u}(q)$. From (4.4) it follows that $h$ extends continuously to a homeomorphism $h: W^{u}(q) \rightarrow W^{u}(\bar{q})$ defined by

$$
d_{\bar{q}}(h(v), \tilde{q})=c \frac{\left(\Delta_{z}\right)^{\delta}}{\Delta_{\bar{z}}}\left[d_{q}(v, q)\right]^{\delta} .
$$

From (M1) we have that $h \mid W^{u}(q)$ is a conjugacy. Of course the map $h: \Sigma \cup W^{u}(q) \rightarrow$ $\bar{\Sigma} \cup W^{u}(\bar{q})$ may not map leaves of $\mathscr{F}_{q}^{s}$ into leaves of $\mathscr{F}_{\bar{q}}^{s}$, but as in the proof of lemma 3.4 one can modify $\mathscr{F}_{\bar{q}}^{s}$ in the complement of a neighbourhood of the orbit of $\bar{z}$ so this is the case. As before we can use these foliations to extend $h$.

Case 1(c): More tangencies of even order; i.e. \#I>1. As before we can induce homeomorphisms $h: \Sigma_{i} \rightarrow \bar{\Sigma}_{i}$ for $i \in I$ and we can define $h$ on $\bigcup_{j} f^{j}\left(\Sigma_{i}\right)$ using $h \circ f=\bar{f} \circ h$. If (M1) holds, then each map $h: \Sigma_{i} \rightarrow \bar{\Sigma}_{i}$ induces a conjugacy $h: W^{u}(q) \rightarrow$ $W^{u}(\bar{q})$ satisfying:

$$
d_{\bar{q}}(h(v), \bar{q})=c \cdot \frac{\left(\Delta_{z_{i}}\right)^{\delta}}{\Delta_{\bar{z}_{i}}} \cdot\left[d_{\bar{p}}(v, q)\right]^{\delta} .
$$

These conjugacies only coincide if (M2) holds. As before one needs to modify $\mathscr{F}_{\bar{q}}^{s}$ so that $h$ maps leaves of $\mathscr{F}_{q}^{s}$ into leaves of $\mathscr{F}_{\bar{q}}^{s}$. Extend $h$ as before.

Case 2. Suppose we are in the same situation as before except that there is exactly one saddle-point $P$ with $W^{u}(P) \cap W^{s}(p) \neq \varnothing$ and exactly one saddle point $Q$ with $W^{s}(Q) \cap W^{u}(q) \neq \varnothing$. Assume also that $W^{u}(P) \cap W^{s}(p)\left(\operatorname{resp} . W^{s}(Q) \cap W^{u}(q)\right)$ is a unique orbit $\mathcal{O}(x)$ (resp. $\mathcal{O}(y)$ ). Suppose that all intersections of invariant manifolds (except of $W^{u}(p)$ and $W^{s}(q)$ ) are transversal, see figure 4.2 .

We will now show how to construct conjugacies in this case. Take $C^{1}$ foliations $\mathscr{F}_{P}^{u}$ and $\mathscr{F}_{\mathrm{Q}}^{\mathrm{s}}$. According to the first theorem in the appendix this is possible. Now in the constructions we made in cases $1(\mathrm{~b})$ and $1(\mathrm{c})$ we had to modify the foliation $\mathscr{F}_{q}^{s}$. But since we do not wish to modify the foliation $\mathscr{F}_{Q}^{s}$ we will have to be careful. In fact here we need condition 4 in the definition in the class $\mathcal{M}$, see $\S 1$. Consider the 


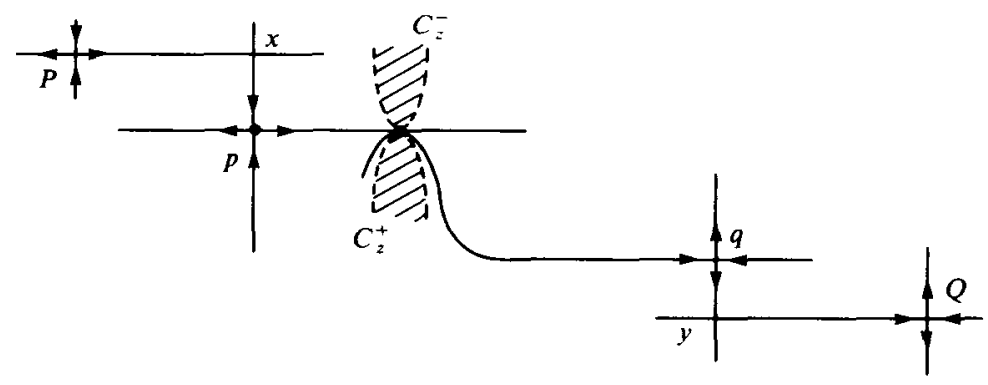

Figure 4.2

cone-like sets $C_{z_{i}}$ as in $\S 3(\mathrm{a})$. Let $C_{z_{i}}^{+}$be the component of $C_{z_{i}} \backslash\left\{z_{i}\right\}$ such that $C_{z_{i}}^{+}$ is disjoint from $W^{u}(P)$, and $C_{z_{i}}^{-}$be the other component. From condition 4 in $\S 1$ it follows that $C_{z_{i}}^{+}$is non-empty and $C_{z_{i}}^{-}$is disjoint from $W^{s}(Q)$. Let $W_{z_{i}^{+}}^{s}(p)$ be the component of $W^{s}(p)-\{p\}$ on to which $f^{n}\left(C_{z_{\mathrm{i}}}^{+}\right)$accumulates and $W_{z_{i}^{-}}^{s}(p)$ the other component of $W^{s}(p)-\{p\}$. Condition 4 implies that $W_{z_{i}^{+}}^{s}(p)$ does not depend on the tangency point $z_{i}$. We also denote this set by $W_{+}^{s}(p)$. Similarly define $W_{z}^{u}(q)$ and $W_{z}^{s}-(q)$. Clearly the foliation $\mathscr{F}_{P}^{u}$ is disjoint from $C_{z_{i}}^{+}$and the foliation $\mathscr{F}_{Q}^{s}$ is disjoint from $C_{z_{i}}^{-}$. So we can modify $\mathscr{F}_{p}^{u}$ in $C_{z_{i}}^{+}$and $\mathscr{F}_{q}^{s}$ in $C_{z_{i}}^{-}$as in $\S 3(\mathrm{a})$, without changing $\mathscr{F}_{P}^{u}$ or $\mathscr{F}_{\mathrm{Q}}^{s}$. In this way we get foliations $\mathscr{F}_{p}^{u}$ and $\mathscr{F}_{q}^{s}$ which either are tangent along a curve $\Sigma_{i}$ (if the tangency at $z_{i}$ is of even order) or only at $z_{i}$ (if the tangency of $W^{u}(p)$ and $W^{s}(q)$ is of odd order). Let us now construct conjugacies.

Case 2(a): \#I=0. The construction is basically the same as in case $1(a)$. We start now by defining conjugacies $h: W^{s}(P) \rightarrow W^{s}(\bar{P})$ and $h: W^{u}(Q) \rightarrow W^{u}(\bar{Q})$. Via the leaves of $\mathscr{F}_{P}^{u}$ and $\mathscr{F}_{Q}^{s}$ this induces homeomorphisms on subsets of $W^{s}(p)$ into $W^{s}(\bar{p})$ and on subsets of $W^{u}(q)$ into $W^{u}(\bar{q})$. Extend these homeomorphisms to conjugacies $h: W^{s}(p) \rightarrow W^{s}(\bar{p})$ and $h: W^{u}(q) \rightarrow W^{u}(\bar{q})$. Now proceed as before.

Case 2(b): $\# I=1$. Let $\Sigma$ be the curve of tangency of $\mathscr{F}_{p}^{u}$ and $\mathscr{F}_{q}^{s}$, and let $\Sigma_{-}$be the component of $\Sigma-\{z\}$ such that $f^{j}\left(\Sigma_{-}\right)$accumulates on $W_{-}^{s}(p)\left(=W_{z}^{s}-(p)\right)$. Now define $h: W_{-}^{s}(p) \rightarrow W_{-}^{s}(\bar{p})$ as in equation (4.1) where we choose $c>0$ such that $h(x)=\bar{x}$, where $x \in W^{s}(p) \cap W^{u}(P)$ and $\bar{x} \in W^{s}(\bar{p}) \cap W^{u}(\bar{P})$. That is:

$$
c=\frac{d_{\bar{p}}(\bar{x}, \bar{p})}{\left(d_{p}(x, p)\right)^{\delta}} .
$$

The map $h$ induces a map on the space of leaves of $\mathscr{F}_{p}^{u}$ and therefore a map $h: \Sigma_{-} \rightarrow \bar{\Sigma}_{-}$. As in case $1(\mathrm{~b})$ this induces a map $h: W_{-}^{u}(q) \rightarrow W_{-}^{u}(\bar{q})$, which is a conjugacy if and only if condition (M1) is satisfied. So up to now we have defined $h$ on one side only: starting in $W_{-}^{s}(p)$ and ending in $W_{-}^{u}(q)$. Similarly one can start in $W_{+}^{u}(q)$ and end in $W_{+}^{s}(p)$. It follows now from the construction and equation (4.1) that $h: W^{s}(p) \rightarrow W^{s}(\bar{p})$ and $h: W^{u}(q) \rightarrow W^{u}(\bar{q})$ are $C^{1}$ outside $p$ and $q$. From this it follows that $h$ extends to $h: W^{s}(P) \rightarrow W^{s}(\bar{P})$ and $h: W^{u}(Q) \rightarrow W^{u}(\bar{Q})$ if and only if

$$
A=\bar{A}, \quad B=\bar{B},
$$


see lemma 3.4. As in the proof of lemma 3.4 one has to change the foliations $\mathscr{F}_{\bar{P}}^{u}$ (resp. $\mathscr{F}_{\tilde{Q}}^{s}$ ) in order to make sure that $h$ maps leaves of $\mathscr{F}_{P}^{u}$ (resp. $\mathscr{F}_{Q}^{s}$ ) into leaves of $\mathscr{F}_{\bar{P}}^{u}$ (resp. $\mathscr{F}_{\bar{Q}}^{s}$ ). As before one extends $h$.

Case 2(c): \#I>1. One can construct conjugacies in this case in the same way as in cases $2(\mathrm{~b})$ and $1(\mathrm{c})$. As in case 1(c) one needs the additional modulus conditions (M2).

Proof of theorem 4.1: Remaining cases. Suppose now that $f$ and $\bar{f}$ are again as in case 2 , but that there is more than one saddle-point $P_{i}$ with $W^{u}\left(P_{i}\right) \cap W^{s}(p) \neq \varnothing$. Let us first treat the case that there is no saddle-point $Q$ with $W^{s}(Q) \cap W^{u}(q) \neq \varnothing$. Then one can modify $\mathscr{F}_{q}^{s}$ so that $\mathscr{F}_{p}^{u}$ and $\mathscr{F}_{q}^{s}$ are tangent only in curves $\Sigma_{i}$ or at the points $z_{i}$. Now construct $h$ as in case 2 . There is one problem left. There are several orbits of intersections $O\left(x_{i}\right) \subset W^{u}\left(P_{i}\right) \cap W^{s}(p)$. But in the construction of $h: W^{s}(p) \rightarrow W^{s}(\bar{p})$ one has to choose a constant $c>0$ as in equation (4.5) so that $h\left(x_{i}\right)=\bar{x}_{i}$ for all $i$. This is possible if and only if

$$
\frac{d\left(x_{i}, p\right)}{d\left(x_{1}, p\right)}=\left\{\frac{d\left(\bar{x}_{i}, \bar{p}\right)}{d\left(\bar{x}_{1}, \bar{p}\right)}\right\}^{\delta}, \quad \text { for all } i .
$$

Since $h \mid W^{s}(p)$ is differentiable we need (M3) in order to extend $h$ to $W^{s}\left(P_{i}\right)$. As before $h \mid W^{s}\left(P_{i}\right)$ must be a linear map (because $\left.A_{i}=\bar{A}_{i}\right)$. If $W^{u}\left(P_{i}\right) \cap W^{s}(p)$ contains more than one orbit we will need (M5) because each orbit will induce a linear map $W^{s}\left(P_{i}\right) \rightarrow W^{s}\left(\bar{P}_{i}\right)$ and these maps must coincide. If all these modulus conditions are satisfied one can extend $h$ as before.

The general case goes similarly. If there are also saddle-points $Q_{j}$ with

$$
W^{s}\left(Q_{j}\right) \cap W^{u}(q) \neq \varnothing
$$

then it follows from $f \in \mathcal{M}$ that $W_{+}^{u}(q) \neq W^{u}(q), W_{+}^{s}(p) \neq W^{s}(p)$ and either

(i) $W^{u}\left(P_{i}\right) \cap W_{+}^{s}(p) \neq \varnothing$ and $W^{s}\left(Q_{j}\right) \cap W_{+}^{u}(q) \neq \varnothing$; or

(ii) $W^{u}\left(P_{i}\right) \cap W_{-}^{s}(p) \neq \varnothing$ and $W^{s}\left(Q_{j}\right) \cap W_{-}^{u}(q) \neq \varnothing$.

In this case one modifies $\mathscr{F}_{p}^{u}$ on one side of $z_{i}$ and $\mathscr{F}_{q}^{s}$ on the other side, see case 2(b). The construction of a conjugacy goes exactly as before. This finishes the proof of theorem (4.1).

\section{Construction of conjugacies: general case}

First we consider the case that:

$5(\mathrm{a}) . W^{u}(p), W^{s}(q)$ and $W^{u}(q), W^{s}(r)$ have non-transversal intersections. All other invariant manifolds intersect transversally. Assume that there are three hyperbolic periodic points $p, q, r$ so that both $W^{u}(p)$ and $W^{s}(q)$ as well as $W^{u}(q)$ and $W^{s}(r)$ have exactly one orbit of non-transversal intersections. As in lemma 2.7 there are exactly four cases A, B, C and D to consider. Let (M1)-(M6) be the modulus conditions from lemma 2.7. We will prove:

THEOREM 5.1. Let $f, \bar{f} \in \mathcal{M}$ be as above and $C^{\infty}$ near each other. Assume that no other invariant manifold of saddle point intersects $W^{s}(q) \cup W^{u}(q)$. The diffeomorphisms $f$ and $\bar{f}$ are conjugate if and only if the modulus conditions (Mi) from lemma 2.7. (i.e. 6,5 or 4 conditions depending on the case we are looking at from lemma 2.7) are satisfied. 
Proof of theorem 5.1. In figure 5.1 we have drawn the situations A, B, C and D. The necessity of the modulus conditions (Mi) are proved in lemma 2.6. As before the numbers $\alpha, \beta, a, b$ denote the eigenvalues at $p, q, r$ as indicated in this figure. We will assume that $\alpha, \beta a, b$ are positive. The general case can be dealt with similarly.

Also we can assume that the tangency of $W^{u}(p)$ and $W^{s}(q)$ and the tangency of $W^{u}(q)$ and $W^{s}(r)$ are of even order, because if one of these tangencies is of odd order, then one can construct the conjugacy as in the proof of theorem 4.1. Finally we will assume that $W^{u}(p) \cap W^{s}(q)$ and $W^{u}(q) \cap W^{s}(r)$ both consist of only one orbit. The general case is treated similarly.

Case A: Denote by $R$ the upper right hand quadrant near $q$, bounded by $W^{u}(q)$ and $W^{s}(q)$. That is, let $U_{q}$ be a rectangular region on which $f$ is linearisable. Then $R$ is the connected component of $U_{q}-W^{s}(q) \cup W^{u}(q)$ having the orbits of tangencies in its boundary. First choose $C^{1}$ invariant foliations $\mathscr{F}_{q}^{s}$ and $\mathscr{F}_{q}^{u}$ having $C^{1}$ tangent line fields. These foliations $\mathscr{F}_{q}^{s}$ and $\mathscr{F}_{q}^{u}$ in'duce a $C^{1}$-linearising system for $f$. Let $d$ be the metric induced by this coordinate system.

(a) Let $W^{u}(p), W^{s}(q)$ (resp. $W^{u}(q)$ and $W^{s}(r)$ ) have a tangency of order $n$ (resp. $m$ ), with $n$ and $m$ even numbers. Define

$$
Q_{x}=\lim _{\substack{z \in W^{s}(q) \\ z \rightarrow x}} \frac{d\left(z, W^{u}(p)\right)}{\{d(z, x)\}^{n}},
$$

and define $Q_{y}$ similarly. In lemma 2.6 it is shown that the numbers

$$
\frac{Q_{x} \cdot[d(x, q)]^{n}}{d(y, q)}, \frac{Q_{y} \cdot\{d(y, q)\}^{m}}{d(x, q)}, \alpha, \beta, a, b
$$

are topological invariants. Let us show that these invariants are sufficient.

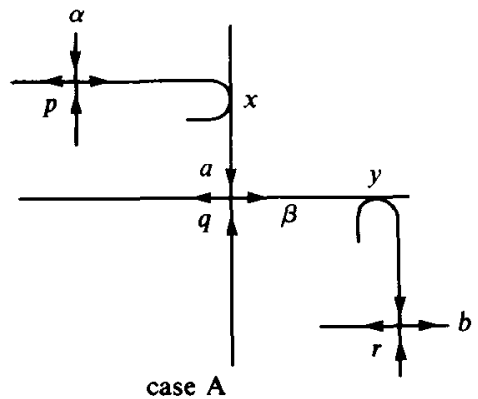

case A

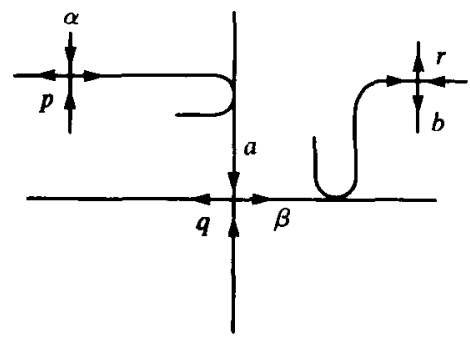

case B
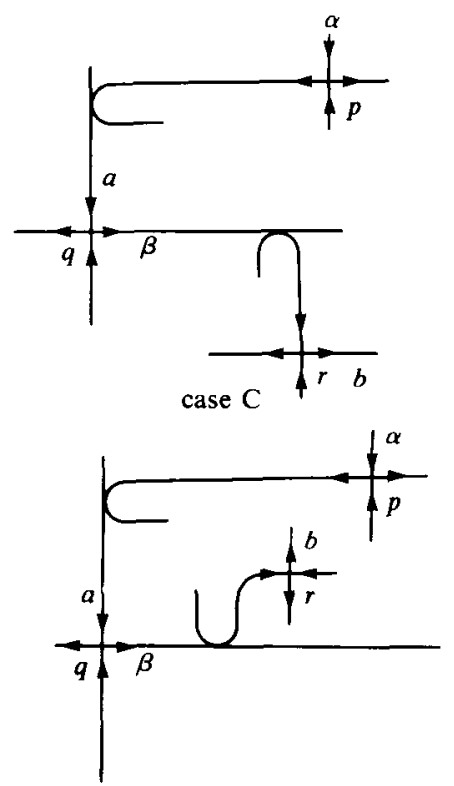

case D

FIGURE 5.1 
(b) So we need to define a conjugacy between $f$ and $\vec{f}$. First we define a conjugacy $h$ on the quadrant $R$. We do this so that $h$ is linear with respect to the linearising coordinates and so that $h(x)=\bar{x}, h(y)=\bar{y}$. Since

$$
a=\bar{a}, \quad \beta=\bar{\beta}
$$

then $h$ indeed defines a conjugacy on $R$ near $q$.

(c) Let us now show that we can extend $h$ to a neighbourhood of $p$, provided $\alpha=\bar{\alpha}$ and the modulus condition on $Q_{x}$ and $Q_{y}$ is satisfied.

Let $l$ be the line through $x$ which is horizontal with respect to the linearising coordinates, and let $\bar{l}$ be similar. Clearly $h(l)=\bar{l}$ and for $x_{i} \rightarrow x$,

$$
\begin{aligned}
\frac{d\left(h\left(x_{i}\right), W^{s}(\bar{q})\right.}{d\left(x_{i}, W^{s}(q)\right)} & \rightarrow \frac{\bar{d}(\bar{y}, \bar{q})}{d(y, q)}, \\
\frac{\bar{d}\left(h\left(x_{i}\right), l\right)}{d\left(x_{i}, l\right)} & \rightarrow \frac{\bar{d}(\bar{x}, \bar{q})}{d(x, q)},
\end{aligned}
$$

where $d, \bar{d}$ are induced by linearising coordinates at $q$ and $\bar{q}$. Take any unstable invariant foliations $\mathscr{F}_{p}^{u}$ and $\mathscr{F}_{\bar{p}}^{u}$. We claim that we can modify $\mathscr{F}_{\bar{p}}^{u}$ so that $\mathscr{F}_{\bar{p}}^{u}$ agrees with $h\left(\mathscr{F}_{p}^{u}\right)$ inside $R$. In fact from lemma $3.5, \S 3(\mathrm{~b})$, it follows that $h$ extends to a conjugacy near $p$ if and only if

$$
\alpha=\bar{\alpha}, \quad Q_{x}=\frac{C_{1}^{n}}{C_{2}} \cdot Q_{\bar{x}},
$$

where $C_{1}, C_{2}$ are respectively the limits in equations (5.4) and (5.3). Clearly this corresponds to two equations from (5.1). From lemma 3.5 it follows that one can extend $h$ to a neighbourhood of $p$, and after a slight modification of $\mathscr{F}_{\bar{p}}^{u}$, the conjugacy $h$ maps leaves of $\mathscr{F}_{p}^{u}$ onto $\mathscr{F}_{\bar{p}}^{u}$.

(d) In the same way one can extend $h$ to a conjugacy near $r$, provided $b=\bar{b}$ and the conjugacy condition on $Q_{y}$ and $Q_{\bar{y}}$ is satisfied.

(e) Now one can extend $h$ as in $\S 4$.

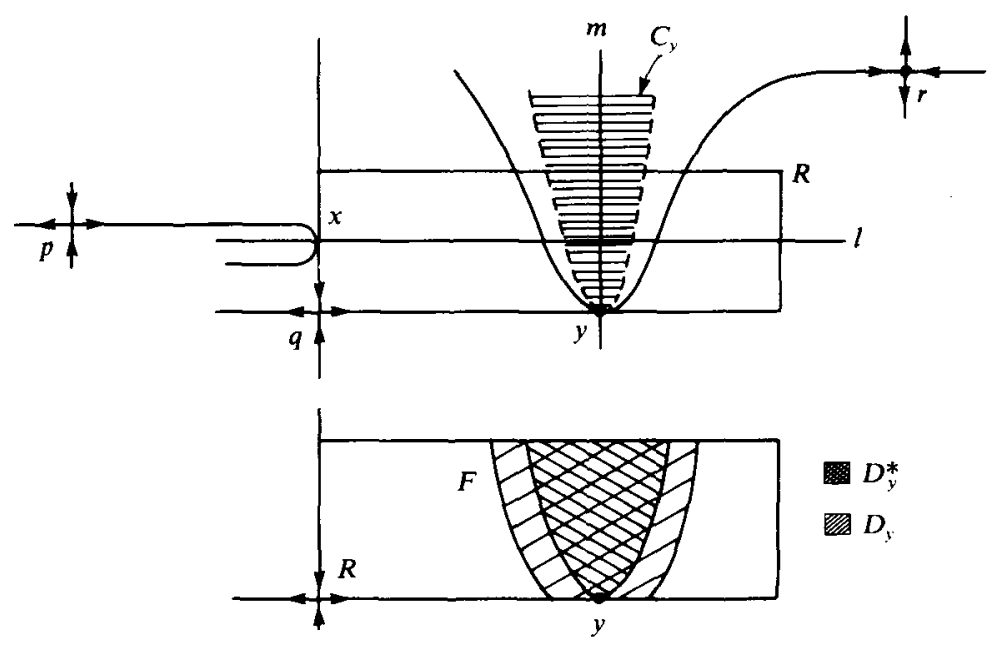

Figure 5.2. 
Remark. If there are invariant manifolds intersecting with $W^{s}(q)$ and $W^{u}(q)$ then one has new necessary moduli conditions, see $\$ 2$ and theorem 4.1. As in $\S 4$ one can extend $h$ to $M$ provided all these additional moduli conditions are satisfied.

Cases B and C. Cases B and C are dual since one obtains case B out of case C by taking $f^{-1}$. Therefore we will just treat case B. In lemma 2.6 it is shown that the numbers

$$
Q_{x} \cdot \frac{\{d(x, q)\}^{n}}{d(y, q)}, \alpha, \beta, a, b
$$

are topological invariants. Since we do not have any moduli conditions on $Q_{y}$ and $Q_{\bar{y}}$ the construction of a conjugacy is more complicated than in the previous case.

(a) As in the previous case choose $C^{1}$-invariant foliations $\mathscr{F}_{q}^{s}$ and $\mathscr{F}_{q}^{u}$ having $C^{1}$ tangent line fields. Let $l$ be the leaf of $\mathscr{F}_{q}^{u}$ through $x$ and $m$ the leaf of $\mathscr{F}_{q}^{s}$ through $y$, see figure 5.2 .

(b) We want to change $\mathscr{F}_{r}^{s}$ so that $\mathscr{F}_{r}^{s}$ and $\mathscr{F}_{q}^{u}$ have only tangencies at $\mathrm{m}$. So consider a cone-like set $C_{y}$ near $y$ as in lemma 3.3, $\$ 3(\mathrm{a})$. From this lemma and the first theorem in the appendix it follows that $\mathscr{F}_{r}^{s}$ and $\mathscr{F}_{q}^{u}$ can only have tangencies inside the sets $f^{i}\left(C_{y}\right)$. Now modify $\mathscr{F}_{r}^{s}$ inside $C_{y}$ so that $\mathscr{F}_{r}^{s}$ restricted to $C_{y}$ is given by homogeneous polynomials (+constants) in terms of the coordinates induced by $\mathscr{F}_{q}^{s}$ and $\mathscr{F}_{q}^{u}$. From lemma 3.2 this is possible. It follows that $\mathscr{F}_{r}^{s}$ and $\mathscr{F}_{q}^{u}$ have only tangencies along $f^{i}(m)$.

(c) Let $R$ be as before. Take a leaf $F$ of $\mathscr{F}_{r}^{s}$ as in figure 5.2 and let $D_{y}$ (resp. $D_{y}^{*}$ ) be the region bounded by $F$ and $\partial R$ (resp. $W^{s}(r)$ and $\partial R$ ). Take $F$ and $R$ so that the sets $f^{i}\left(D_{y}\right)$ are mutually disjoint. Now let $\mathscr{F}^{s}$ be an invariant foliation on $R$ which is identical to $\mathscr{F}_{r}^{s}$ in $\bigcup f^{i}\left(D_{y}\right)$ and identical to $\mathscr{F}_{q}^{s}$ away from $\bigcup f^{i}\left(D_{y}\right)$. By lemma 3.3 one can choose the rectangle $R$ and $\mathscr{F}^{s}$ so that all its leaves are transversal to $\mathscr{F}_{q}^{u}$ outside the cones $f^{i}\left(C_{y}\right)$. From the construction of $\mathscr{F}_{r}^{s}$ and since $\mathscr{F}^{s}$ coincides with $\mathscr{F}_{r}^{s}$ inside $D_{y}, \mathscr{F}^{s}$ and $\mathscr{F}_{q}^{u}$ are tangent precisely along iterates of $m$.

(d) Now we define the conjugacy on $R$. First define linear conjugacies $h: W^{s}(q) \rightarrow$ $W^{s}(\bar{q})$ and $h: W^{u}(q) \rightarrow W^{u}(\bar{q})$ so that $h(x)=\bar{x}$ and $h(y)=\bar{y}$, see equation (4.2). Since $\beta=\bar{\beta}$ and $a=\bar{a}$ this is possible. These maps induce maps on the space of leaves of $\mathscr{F}_{q}^{u}$ and of $\mathscr{F}^{s}$ outside iterates of $D_{y}^{*}$. Therefore these maps induce homeomorphisms $h: m \rightarrow \bar{m}$ and $h: \Lambda D^{+} \rightarrow \vec{l} \backslash \bar{D}^{+}$, where $D^{+}=\bigcup f^{i}\left(D_{y}^{*}\right)$ and $\bar{D}^{+}=$ $\cup f^{i}\left(\bar{D}_{y}^{*}\right)$. Since $\mathscr{F}_{q}^{u}$ and $\mathscr{F}^{s}$ are precisely tangent along (iterates of) $m$, the homeomorphism $h: m \rightarrow \bar{m}$ induces also a homeomorpinism $h: l \cap D^{+} \rightarrow l \cap \bar{D}^{+}$, via the leaves of $\mathscr{F}^{s}$. Hence there is a unique homeomorphism $h$ on $\operatorname{int}(R)$ which preserves $\mathscr{F}_{q}^{u}$ and $\mathscr{F}^{s}$. Let us check that $h$ is a continuous conjugacy. In order to do this we want to apply lemma 3.5 as in the previous case, $\mathrm{A}$. We have to make some estimates similar to (5.3) and (5.4).

LEMMA 5.2. Take metrics $d, \bar{d}$ corresponding to the linearising coordinates for $f$ and $\bar{f}$ 
near $q$ and $\bar{q}$. Then one has for a sequence $x_{i} \rightarrow x$,

$$
\left.\begin{array}{rl}
\frac{\bar{d}\left(h\left(x_{i}\right), \bar{l}\right)}{d\left(x_{i}, l\right)} & \rightarrow \frac{\bar{d}(\bar{x}, \bar{q})}{d(x, q)}, \\
\frac{\bar{d}\left(h\left(x_{i}\right), W^{s}(\bar{q})\right)}{\bar{d}\left(x_{i}, W^{s}(q)\right)} & \rightarrow \frac{\bar{d}(\bar{y}, \bar{q})}{d(y, q)} .
\end{array}\right\}
$$

Proof. The first limit follows from the fact that $h$ preserves $\mathscr{F}_{q}^{u}$ and from the fact that $h \mid W^{s}(q)$ is linear with $h(x)=\bar{x}$. The second limit is proved as follows. If $x_{i} \rightarrow x$ and $f^{j(i)}\left(x_{i}\right) \rightarrow w \in W^{u}(q)$, then

$$
\begin{gathered}
d\left(x_{i}, W^{s}(q)\right) \simeq|\beta|^{j(i)} \cdot d(w, q), \\
\bar{d}\left(h\left(x_{i}\right), W^{s}(\bar{q})\right) \simeq|\bar{\beta}|^{j(i)} \cdot \bar{d}(h(w), \bar{q}) .
\end{gathered}
$$

Since $h \mid W^{u}(q)$ is linear and $h(y)=\bar{y}$ the second equation in (5.5) follows by taking the ratio of the two equations in (5.6).

Now it follows from equations (5.5) and the modulus conditions on $\alpha, Q_{x}$, that $h$ can be extended to a conjugacy near $p$. This is done exactly as in case A.

(e) As before one can extend $h$ to $M$. In fact since $h$ maps $\mathscr{F}^{s}$ into $\bar{F}^{s}$ it follows from lemma 3.4 in $\S 3(\mathrm{~b})$, and from $b=\bar{b}$ that $h$ extends to conjugacy near $r$. The extension to $M$ goes as before.

Case D. In lemma 2.6 it is shown that in this case the numbers

$$
\alpha, \beta, a, b
$$

are topological invariants. Now we show that there are no modulus conditions on $Q_{x}$ and $Q_{y}$ in this case. It follows that any conjugacy $h$ between $f$ and $\bar{f}$ will be highly non-linear in this case. We construct the conjugacy in a number of steps:

(a) As before take $C^{1}$-unstable and stable foliations $\mathscr{F}_{q}^{u}$ and $\mathscr{F}_{q}^{s}$.

(b) Take a region $D_{y}$ as in the previous case. Here the boundary of $D_{y}$ is a leaf of $\mathscr{F}_{r}^{s}$ (or is a piece of $\partial R$ ). Let $N_{y}$ be a neighbourhood of $D_{y}$. By lemma 3.3 all the leaves of $\mathscr{F}_{r}^{s}$ are transversal to the leaves of $\mathscr{F}_{q}^{s}$ and $\mathscr{F}_{q}^{u}$ inside $N_{y} \backslash C_{y}$. Hence, using the implicit function theorem we can take a new invariant foliation $\mathscr{F}_{q}^{s^{\prime}}$ for $q$ (which is $C^{1}$ except possibly at iterates of $y$ and identical to $\mathscr{F}_{q}^{s}$ outside iterates of $N_{y} \backslash C_{y}$ ) so that each leaf of $\mathscr{F}_{r}^{s}$ in $D_{y} \backslash C_{y}$ is the graph of a homogeneous polynomial function (+constants) with respect to the coordinates induced by $\mathscr{F}_{q}^{s^{\prime}}$ and $\mathscr{F}_{q}^{u}$. Take a similar set $D_{x}$ and a similar foliation $\mathscr{F}_{q}^{u}$ related to $\mathscr{F}_{p}^{u}$.

(c) Modify the foliations $\mathscr{F}_{p}^{u}$ and $\mathscr{F}_{r}^{s}$ as before. That is, modify $\mathscr{F}_{r}^{s}$ inside $C_{y}$ so that the leaves are given by homogeneous polynomials with respect to the $C^{1}$ linearising coordinates induced by $\mathscr{F}_{q}^{s^{\prime}}$ and $\mathscr{F}_{q}^{u^{\prime}}$. Do the corresponding modification for $\mathscr{F}_{\mathrm{r}}^{s}$.

(d) Now take an invariant foliation $\mathscr{F}^{s}$ as before which is identical to $\mathscr{F}_{r}^{s}$ inside $D_{y}$ and identical to $\mathscr{F}_{q}^{s}$ away from iterates of $D_{y}$. Take a similar foliation $\mathscr{F}^{u}$. According to lemma 3.3 and the last theorem in the appendix all tangencies of $\mathscr{F}^{s}$ and $\mathscr{F}^{u}$ are contained in $f^{i}\left(C_{x}\right) \cup f^{j}\left(C_{y}\right)$. Let us study these tangencies.

(e) First we study the tangencies of $\mathscr{F}^{s}$ and $\mathscr{F}^{u}$ in $f^{i}\left(C_{x}\right) \cap f^{j}\left(C_{y}\right)$. Since $\mathscr{F}^{s}$ and $\mathscr{F}^{u}$ are given by polynomial functions it suffices to prove the following lemma. 


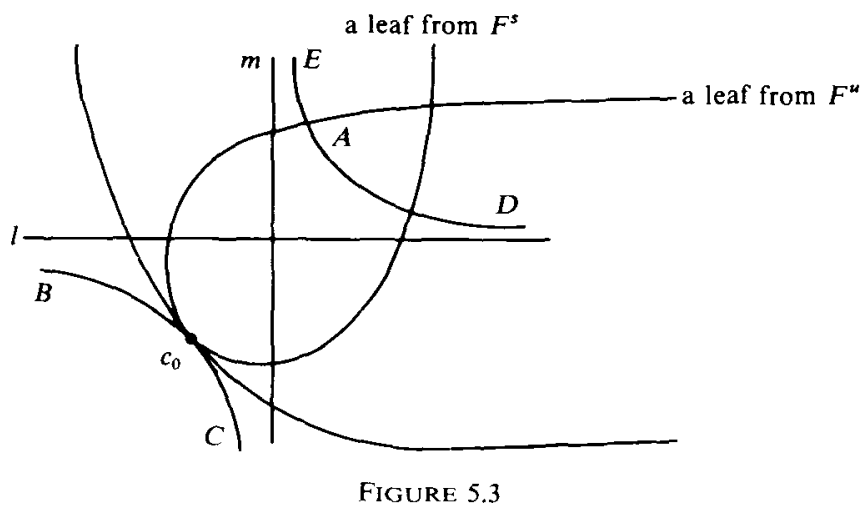

LEMMA 5.3. Consider the following two foliations in $\mathbb{R}^{2}$ :

$$
\begin{array}{ll}
F^{u}=\left\{(u, v) \mid u=Q_{1} \cdot v^{n}+a,\right. & a \in \mathbb{R}\} \\
F^{s}=\left\{(u, v) \mid v=Q_{2} \cdot u^{m}+b,\right. & b \in \mathbb{R}\},
\end{array}
$$

where $n, m$ are even. Then:

(i) $F^{s}$ and $F^{u}$ are tangent along a curve consisting of two components;

(ii) there is a unique point $c_{0}$ where $F^{s}$ and $F^{u}$ have a tangency of odd order. $F^{s}$ and $F^{u}$ are transversal to $c$ except at $c_{0}$.

Proof. If $F^{s}$ and $F^{u}$ are tangent at $(u, v)$ then the tangency vector $\left(1, m Q_{2} u^{m-1}\right)$ to $F^{s}$ at $(u, v)$ and the tangency vector $\left(n Q_{1} v^{n-1}, 1\right)$ to $F^{u}$ are proportional. Hence $F^{s}$ and $F^{u}$ are tangent along the curve:

$$
n \cdot m \cdot Q_{1} \cdot Q_{2} \cdot u^{m-1} \cdot v^{n-1}=1 .
$$

Statement (ii) follows similarly.

We have drawn the curve $c$ as a union of the curves A, B, C, D, E in figure (5.3). We can also make the following:

Observations. (1) Each leaf of $F^{s}$ and each leaf of $F^{u}$ intersects the curve $c$ at least once and at most three times.

(2) There is a curve $A$ in $c$, as drawn in figure 5.3 , so that if a leaf in $F^{s}$ or a leaf in $F^{u}$ intersects $A$ then this leaf has no other intersections with $c$.

Let $A, B, C, D, E$ be the curves in $c$ as is shown in figure 5.3.

From the lemma it follows that all the tangencies of $\mathscr{F}^{u}, \mathscr{F}^{s}$ inside $f^{i}\left(C_{x}\right) \cap f^{-j}\left(C_{y}\right)$ are subsets of (scaled down) copies $c_{i j}$ of the curve $c$ as above. The last theorem in the appendix implies that the leaves of $\mathscr{F}^{u}$ (respectively $\mathscr{F}^{s}$ ) outside $C_{y}\left(C_{x}\right)$ accumulate in a $C^{1}$ sense to $W^{s}(q)\left(W^{u}(q)\right)$. Hence the set $c_{i j}$ consists of two components, and contains the point $c_{0}$ and the arc $A$.

(f) Since $\mathscr{F}_{a}^{u^{\prime}}$ is equal to $\mathscr{F}_{q}^{u}$ in $C_{x}$ it follows that inside $f^{i}\left(C_{x}\right) \cap f^{-j}\left(D_{y}\right)$ the foliations $\mathscr{F}^{s}, \mathscr{F}^{u}$ are polynomial with respect to $\mathscr{F}_{q}^{u}$ and $\mathscr{F}_{q}^{s}$. Hence inside these sets the tangencies of $\mathscr{F}^{u}$ and $\mathscr{F}^{s}$ are as in (e). Furthermore $\mathscr{F}^{s}$ is identical to $\mathscr{F}_{q}^{s}$ outside the iterates of a neighbourhood $N_{y}$ of $D_{y}$. Hence all tangencies of $\mathscr{F}^{s}$ with $\mathscr{F}^{u}$ outside $f^{j}\left(N_{y}\right)$ are contained in the lines $f^{i}(l)$. Now choose $\mathscr{F}^{s}$ on $N_{y} \backslash D_{y}$ so 

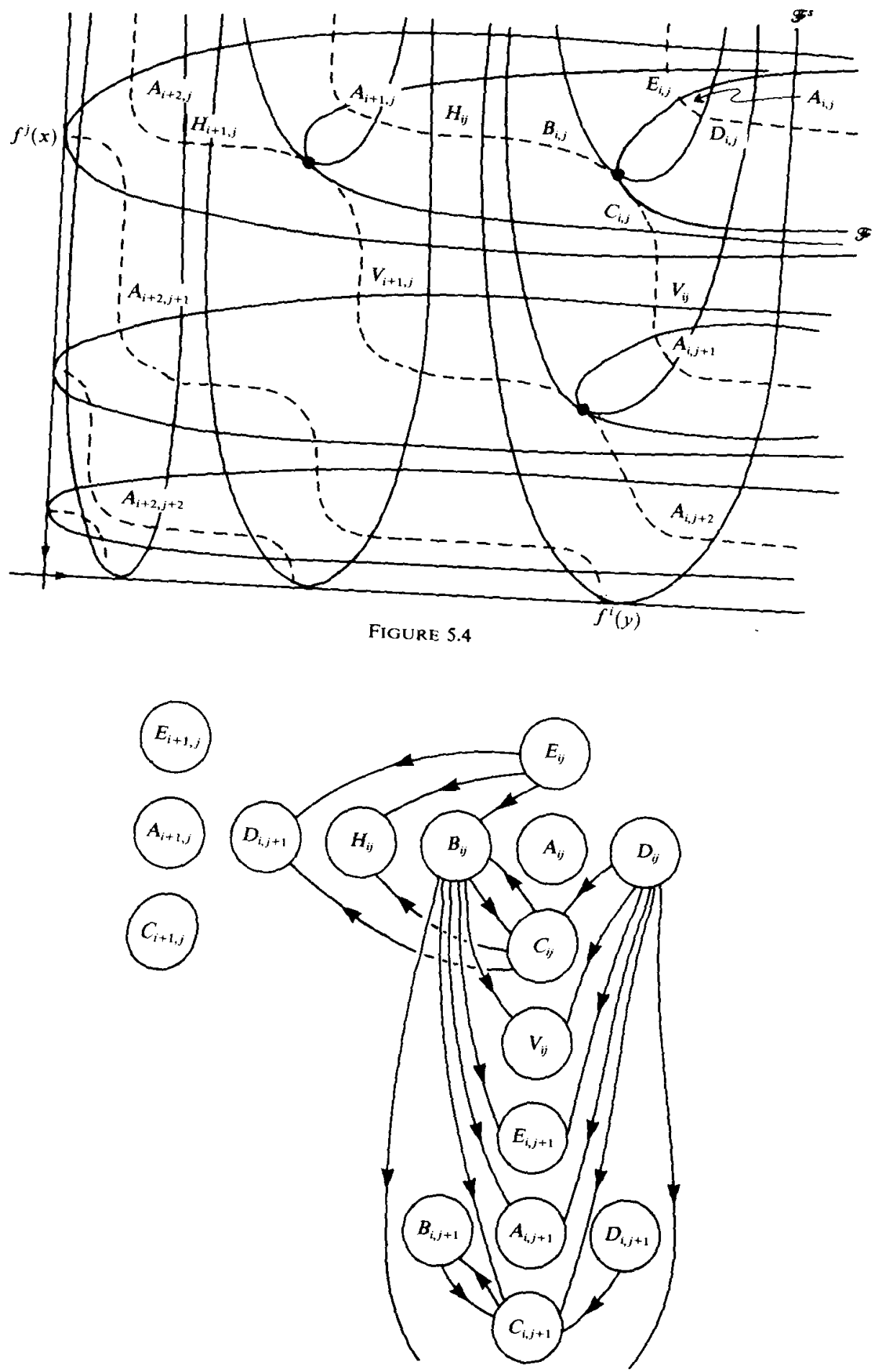

Figure 5.5 
that $\mathscr{F}^{s}$ has only tangencies with $\mathscr{F}^{u}$ in $\left(N_{y} \backslash D_{y}\right) \cap f^{i}\left(C_{x}\right)$ along line segments. Similarly inside $f^{-j}\left(C_{y}\right) \cap f^{i}\left(D_{x}\right)$ the foliations $\mathscr{F}^{s}, \mathscr{F}^{u}$ are polynomial w.r.t. $\mathscr{F}_{q}^{u^{\prime}}$ and $\mathscr{F}_{q}^{s}$. Now choose $\mathscr{F}^{u}$ or $N_{x} \backslash D_{x}$ similarly as above. Since all tangencies of $\mathscr{F}^{s}$ and $\mathscr{F}^{u}$ are contained in $f^{i}\left(C_{x}\right) \cup f^{-j}\left(D_{y}\right)$ it follows that $\mathscr{F}^{s}$ and $\mathscr{F}^{u}$ are tangent along a curve $c$ (with a countable number of components), as drawn in figure 5.4.

(g) Now one can decompose the tangency curve $c$ in curves $A_{i j}, B_{i j}, C_{i j}, D_{i j}, F_{i j}$, $H_{i j}, V_{i j}$ as is shown in figure 5.4. Here $A_{i j}$ etc. are contained in $f^{i}\left(C_{x}\right) \cap f^{j}\left(C_{y}\right)$. The curves $H_{i j}, V_{i j}$ are bounded by $W^{s}(r)$ and $W^{u}(p)$, and the curves $A_{i j}, B_{i j}, C_{i j}$, $D_{i j}, E_{i j}$, are as in figure 5.3. Let $l_{i}=f^{i}(l)$ and $m_{j}=f^{-j}(m)$. Using the leaves of these foliations we can define a diagram of maps between those intervals as is shown in figure 5.5. More precisely: to each point $z$ on the tangency curve $c$ we associate a new point $\psi(z) \in c$. We do this as follows.

(i) For $z \in A_{i j}$, let $\psi(z)=z$.

(ii) For $z \in B_{i j}$ take the leaf $F$ of $\mathscr{F}^{s}$ through $z$. Follow $F$ downward and let $\psi(z)$ be the first intersection of $F$ with the curve $c$ in one of the components $C_{i, j^{\prime}}, V_{i, j^{\prime}}$, $E_{i, j^{\prime}}$, or $A_{i j^{\prime}}$, see the diagram in figure 5.5.

(iii) For $z \in C_{i j}$ follow a leaf $F$ of $F^{u}$ to the left and let $\psi(z)$ be the first intersection of $F$ with $c$ in a component $B_{i^{\prime}, j}, H_{i^{\prime}, j}, D_{i^{\prime}, j}$ or $A_{i^{\prime}, j}$.

(iv) $z \in D_{i j}$. Then follow $F$ downwards as in case (ii).

(v) $z \in E_{i j}$ as (iii).

(vi) $z \in V_{i j}$, then let $\psi(z)$ be the intersection of the leaf of $\mathscr{F}^{u}$ through $z$ with $W^{s}(q)$.

(vii) $z \in H_{i j}$, then $\psi(z)$ is the intersection of the leaf of $\mathscr{F}^{s}$ through $z$ with $W^{u}(q)$.

Clearly if $h$ is a conjugacy between two diffeomorphisms $f, \bar{f}$ as above and if $h$ preserves the foliations $\mathscr{F}^{s}, \mathscr{F}^{u}$, then one must have $h(c)=\bar{c}$ and $h \circ \psi=\bar{\psi} \circ h$. From this it follows that we have very little freedom in choosing a conjugacy $h$.

For example $\psi^{-1}$ maps $B_{i j} \cup C_{i j}$ into $C_{i j} \cup B_{i j}$. We will show shortly that this map in fact is a contraction. Clearly for the point $c_{i j} \in C_{i j} \cap B_{i j}$ where $\mathscr{F}^{u}$ and $\mathscr{F}^{s}$ are tangent with $c$, one has $\psi\left(c_{i j}\right)=c_{i j}$. Since $h$ must respect all foliations, it must map the end-points of $B_{i j} \cup C_{i j}$ onto end-points of $\overline{B_{i j}} \cup \overline{C_{i j}}$ since these points belong to stable and unstable manifolds. Hence $h$ is completely fixed in a sequence of points in $B_{i j} \cup C_{i j}$ converging to a fixed point $c_{i j}$ of $\psi$.

Clearly $\psi^{k}(z)$ is either contained in $W^{s}(q), W^{u}(q)$ or $A_{i j}$ for some sufficiently big $k$ or the sequence $\left\{\psi^{k}(z)\right\}$ is infinite. If this sequence is infinite assign to it a symbol-sequence $\left\{S_{k}\right\}$, where $S_{k}$ is the component $B_{i j}, C_{i j}, D_{i j}$ or $E_{i j}$ of $c$ which contains $\psi^{k}(z)$.

LEMMA 5.4(i). If the sequence $\left\{\psi^{k}(z)\right\}$ is infinite then no other point $z^{\prime} \in c$ has the same symbol sequence as $z$.

(ii) If this sequence is finite it must end with an interval $A_{i j}, W^{s}(q)$ or $W^{u}(q)$. Moreover in this case there is a small interval (in the curve c) of points having the same sequence.

Proof. The second statement is obvious from the definitions and by continuity. So suppose the sequence $\left\{\psi^{k}(z)\right\}$ is infinite. Then for each $k \in \mathbb{N}, \psi^{k}(z)$ is contained in intervals $B_{i j}, C_{i j}, D_{i j}, E_{i j}$ for some $i, j$. Let $\pi_{l}$ be the projection from $B_{i j} \cup D_{i j}$ on $f^{i}(l)$ 
defined as follows. Take $z \in B_{i j} \cup D_{i j}$, and the leaf $F$ of $\mathscr{F}^{s}$ through $z$. Let $\pi_{l}(z)$ be the intersection of $F$ with $f^{i}(l)$ (near $z$ ). Similarly define a projection $\pi_{m}$ : $C_{i j} \cup E_{i j} \rightarrow f^{-j}(m)$. Then define

$$
\theta(z)= \begin{cases}\pi_{m} \circ \psi \circ \pi_{l}^{-1}(z) & \text { for } z \in \pi_{l}\left(B_{i j} \cup D_{i j}\right), \\ \pi_{l} \circ \psi \circ \pi_{m}^{-1}(z) & \text { for } z \in \pi_{m}\left(C_{i j} \cup E_{i j}\right) .\end{cases}
$$

Now $f^{i}(l)$ and $f^{-j}(m)$ are all copies of $\mathbb{R}^{+}$so we can consider $\theta$ as a map from $\theta: \mathbb{R}^{+} \rightarrow \mathbb{R}^{+}$(which is not defined everywhere). We claim that $\theta$ is an expansion:

$$
\left|\theta^{\prime}(z)\right|>1 \text {. }
$$

From this claim the lemma follows. In fact take a point $z^{\prime} \in c$ near $z$. If $\psi^{k}\left(z^{\prime \prime}\right)$ is an infinite sequence for every $z^{\prime \prime} \in c$ between $z$ and $z^{\prime}$, then it follows that the length of $\psi^{k}$ (segment on $c$ between $z$ and $z^{\prime}$ ) has finite length. Since $\theta$ is an expansion, this is impossible.

So let us prove (5.7). From the construction above it follows that $A_{i j}, B_{i j}, C_{i j}, D_{i j}, E_{i j}$ are contained in the set

$$
\left\{f^{i}\left(C_{x}\right) \cap f^{-j}\left(D_{y}\right)\right\} \cup\left\{f^{i}\left(D_{x}\right) \cap f^{-j}\left(C_{y}\right)\right\} .
$$

Inside $f^{-j}\left(D_{y}\right) \cap f^{i}\left(C_{x}\right)$ the foliations $\mathscr{F}^{s}, \mathscr{F}^{u}$ are given by polynomials (+constants) w.r.t. $\mathscr{F}_{q}^{u}$ and $\mathscr{F}_{q}^{s^{\prime}}$. Similarly inside $f^{i}\left(D_{x}\right) \cap f^{-j}\left(C_{y}\right)$ the foliations $\mathscr{F}^{s}, \mathscr{F}^{u}$ are polynomial w.r.t. $\mathscr{F}_{q}^{u^{\prime}}$ and $\mathscr{F}_{q}^{s}$. It follows that it is sufficient to prove the following lemma.

LEMMA 5.5. Consider the following two foliations:

$$
\begin{array}{ll}
F^{u}=\left\{(u, v) ; u=Q_{1} \cdot v^{n}+a,\right. & a \in \mathbb{R}\} \\
F^{s}=\left\{(u, v) ; v=Q_{2} \cdot u^{m}+b,\right. & b \in \mathbb{R}\},
\end{array}
$$

where $n, m$ are even. Let $F_{a}^{u}\left(F_{b}^{s}\right)$ be the leaf of $F^{u}\left(F^{s}\right)$ through $(a, 0)($ resp. $(0, b))$. Let $\theta(b)$ be the number $a \in \mathbb{R}$ so that $F_{a}^{u}$ is tangent to $F_{b}^{s}$ in the intervals $C \cup E$, see figure 5.3. Then $\theta: \mathbb{R} \rightarrow \mathbb{R}$ is an expansion, i.e. $\left|\theta^{\prime}\right|>1$. A similar result holds in $B \cup D$, if we change the role of $F^{u}$ and $F^{s}$.

Proof. The foliations $F^{u}$ and $F^{s}$ are tangent in the curve

$$
v=c(u)=\frac{1}{\left(n \cdot m \cdot Q_{1} \cdot Q_{2}\right)^{1 / n-1} \cdot u^{m-1 / n-1}} .
$$

So

$$
\begin{aligned}
& b=c(u)-Q_{2} \cdot u^{m}=B(u), \\
& a=u-Q_{1} \cdot(c(u))^{n}=A(u) .
\end{aligned}
$$

Now $a$ is related to $b$ by:

$$
a=A \circ B^{-1}(b)=\theta(b) .
$$

Hence since $n Q_{1} \cdot(c(u))^{n-1} \cdot m \cdot Q_{2} u^{m-1}=1$ one has

$$
\theta^{\prime}(b)=\frac{A^{\prime}(u)}{B^{\prime}(u)}=\frac{1-Q_{1} \cdot n \cdot(c(u))^{n-1} \cdot c^{\prime}(u)}{c^{\prime}(u)-Q_{2} \cdot m \cdot u^{m-1}}=\frac{1}{m \cdot Q_{2} \cdot u^{m-1}} .
$$

Here the last equality follows by using the definition of $c(u)$ explicitly. For the point where the curve $c$ is tangent to $F^{u}$ and $F^{s}$ one has $m \cdot Q_{2} \cdot u^{m-1}=1$. Since by assumption $F_{a}^{u}$ and $F_{b}^{s}$ are tangent in $C \cup E$ it follows that $\left|\theta^{\prime}(b)\right|>1$. 
(h) Up to now we have investigated the set of tangencies of $\mathscr{F}^{u}$ and $\mathscr{F}^{s}$, and a diagram related to these tangencies. Now we can define the conjugacy $h$ as follows.

Take conjugacies $h: W^{s}(q) \rightarrow W^{s}(q), h: W^{u}(q) \rightarrow W^{u}(q)$ which are linear and so that $h(x)=x$. Since

$$
a=\bar{a}, \quad \beta=\bar{\beta}
$$

this is possible. Then take a homeomorphism

$$
h: A_{i 0} \rightarrow \bar{A}_{i 0} \text {. }
$$

Here we have freedom. Extend $h$ to $\bigcup_{i j} A_{i j}$ by forcing the conjugacy $h \circ f=\bar{f} \circ h$. From lemma 5.4 it follows that there is a unique extension of $h$ to the tangency curve $c$ so that $\bar{\psi} \circ h=h \circ \psi$, i.e. so that $h$ respects the diagram in figure 5.5. It follows that there is a unique extension of $h$ to $R$ which respects the foliations $\mathscr{F}^{s}$ and $\mathscr{F}^{u}$. From the way $h$ is constructed it follows that $h$ is monotone. By interchanging the role of $f$ and $\bar{f}$ it follows that $h$ has a monotone inverse. Hence $h: c \rightarrow \bar{c}$ is a homeomorphism. Since $\mathscr{F}_{q}^{s^{\prime}}$ is a $C^{1}$ foliation, except possibly in $y$, it follows as in lemma 5.2 that

$$
\lim _{\substack{z \rightarrow x \\ z \in l}} \frac{\bar{d}(h(z), x)}{d(z, x)}
$$

converges. Here $d, \bar{d}$ are $C^{0}$-metrics. It follows from lemma 3.4 that $h$ extends to a conjugacy near $p$, since

$$
\alpha=\bar{\alpha} .
$$

Similarly $h$ can be extended to a conjugacy near $r$, since

$$
b=\bar{b} \text {. }
$$

5(b). Construction of conjugacies: remaining cases. Let $f, \bar{f} \in \mathcal{M}$ be as in theorem 5.1 except that there are finitely many saddle-points whose invariant manifolds intersect $W^{s}(q) \cup W^{u}(q)$ transversally in a finite number of orbits. To define a conjugacy $h$ in this case we start by constructing stable and unstable foliations for these saddle points and then we construct the foliations $\mathscr{F}^{s}$ and $\mathscr{F}^{u}$ compatible with these foliations. We then perform the construction of the proof of theorem 5.1. In order to extend this conjugacy to a neighbourhood of the saddle points whose invariants manifolds intersect $W^{s}(q) \cup W^{u}(p)$ we need more moduli conditions as in $\S 4$. If these extra moduli conditions are satisfied we extend the conjugacy using the same arguments of the proof of theorem 4.1.

Another situation that may occur for a diffeomorphism $f \in \mathcal{M}$ having a cascade of tangencies is a combination of the four cases treated in $\S 5(\mathrm{a})$. In fact we may have a finite number of saddle points $p_{1}, \ldots, p_{q}$ such that $W^{u}\left(p_{i}\right)$ has a finite number of orbits of non-transversal intersection with $W^{s}(q)$ and also a finite number of saddles $q_{1}, \ldots, q_{s}$ whose stable manifolds have orbits of non-transversal intersection with $W^{u}(q)$. If $\bar{f}$ is a nearby diffeomorphism having the same intersection pattern of stable and unstable manifolds we can construct a conjugacy between $f$ and $\bar{f}$ by putting the previous techniques together provided the appropriate moduli conditions are satisfied. 


\section{A bound for the number of tangencies}

In $\S 4$ and $\S 5$ we proved that two nearby diffeomorphisms in $\mathcal{M}$, having the same intersection pattern of stable and unstable manifolds, are conjugate provided a finite number of moduli conditions are satisfied. In order to conclude the proof of the main theorem it remains to show that every diffeomorphism $f \in \mathcal{M}$ has a neighbourhood $\mathcal{N}$ which contains a countable number of $k$-parameter families of diffeomorphisms such that any diffeomorphism in $\mathcal{N}$ has the same intersection pattern of stable and unstable manifolds as some diffeomorphism in one of these families. In this section we will achieve this by proving the following result.

THEOREM 6.1. If $f \in \mathcal{M}$ then there exists a neighbourhood $\mathcal{N}$ of $f$ and a number $K>0$ such that $\mathcal{N} \subset \mathcal{M}$ and the number of orbits of non-transversal intersection of stable and unstable manifolds of every $g \in \mathcal{N}$ is at most $K$.

Proof. Since $\mathscr{A}$ is an open set [Sm1], it is easy to see that every $f \in \mathcal{M}$ has a neighbourhood $\mathcal{N} \subset \mathscr{A}$ such that every $g \in \mathcal{N}$ satisfies conditions (1), (3), (4) and (5) of the definition of $\mathcal{M}$ in $\S 1$. Now we prove that if $\mathcal{N}$ is small enough then condition (2) is also satisfied and we get a bound for the number of orbits of tangencies. Let $V \subset M$ be a small open set such that each orbit of tangency of $f$ has a unique point in $V$. So, in order to prove the theorem it is enough to show that for each $g \in \mathcal{N}$, the number of tangencies of $g$ in $V$ is at most $K$ and these tangencies are of finite order. Let $x \in V$ be a point of tangency of order $r$ of $W^{u}(p)$ and $W^{s}(q)$ where $p$ and $q$ are saddle points of $f$. For each $g \in \mathcal{N}$ let $U(g)=$ $W^{u}(p(g)) \cap V$ and $S(g)=W^{s}(q(g)) \cap V$ where $p(g)$ (resp. $q(g)$ ) is the periodic point of $g$ near $p$ (resp. $q$ ). If $P(g)$ is a saddle point of $g$ whose unstable manifold intersect $W^{s}(p(g))$ then, by the $\lambda$-Lemma $[\mathbf{P 1}], W^{u}(P(g)) \cap V$ is a sequence of submanifolds $U_{n}(g)$ which converges to $U(g)$ in the $C^{\infty}$ topology. Similarly if $Q(g)$ is a saddle point whose stable manifold intersects $W^{u}(q(g))$ then $W^{s}(Q(g)) \cap V$ is a sequence of submanifolds $S_{m}(g)$ converging to $S(g)$ in the $C^{\infty}$ topology. Since $f \in \mathcal{M}$ we have that $S_{m}(f)$ is transversal to $U_{n}(f)$ for all $m, n \in \mathbb{N}$ and $U(f) \cap S(f)=$ $\{x\}$. Hence if $\mathcal{N}$ and $V$ are small enough and $z \in U_{n}(g) \cap S_{m}(g)$ then the contact between $U_{n}(g)$ and $S_{m}(g)$ at $z$ is at most $r$. So we need to prove that

$$
\#\left\{m \in \mathbb{N} ; U_{m}(g) \text { is not transversal to } S_{n}(g) \text { for some } n \in \mathbb{N}\right\}
$$

is uniformly bounded.

For each $g \in \mathcal{N}$ let $\mathscr{F}_{p(g)}^{u}$ be a $C^{1}$ unstable foliation at $p(g)$ such that each $U_{n}(g)$ is contained in a leaf of $\mathscr{F}_{p(g)}^{u}$ and the $r$-jet of $\mathscr{F}_{p(g)}^{u}$ is $C^{1}$ and varies continuously with $g$ (see the appendix). Similarly we consider a stable foliation $\mathscr{F}_{q(g)}^{s}$ compatible with $W^{s}(Q(g))$. Let $(u, v)$ be a $C^{\infty}$ coordinate system in a neighbourhood $V_{x}$ of $x$ such that $U(f)=\{(u, 0) ; u \in(-a, a)\}$ and $S(f)=\left\{(u, v) ; v=u^{r}, u \in(-a, a)\right\}$. We may take $V$ and $\mathcal{N}$ so that for each $g \in \mathcal{N}$

$$
\begin{aligned}
U_{n}(g) & =\left\{(u, v) ; v=\psi_{n}^{g}(u)\right\} \\
U(g) & =\left\{(u, v) ; v=\psi_{\infty}^{g}(u)\right\} . \\
S_{m}(g) & =\left\{(u, v) ; v=\varphi_{m}^{g}(u)\right\} \\
S(g) & =\left\{(u, v) ; v=\varphi_{\infty}^{g}(u)\right\}
\end{aligned}
$$


where $\varphi_{m}^{g}, \varphi_{\infty}^{g}, \psi_{n}^{g}, \psi_{\infty}^{g}:[-a, a] \rightarrow \mathbb{R}$ are $C^{\infty}$ functions satisfying the following properties:

(a) The maps $g \mapsto D^{j} \varphi_{m}^{g}, D^{j} \varphi_{\infty}^{g}, D^{j} \psi_{n}^{g}, D^{j} \psi_{\infty}^{g}$, from $\mathcal{N}$ to $C^{1}([-a, a], \mathbb{R})$ are continuous for $j=0,1, \ldots, r$.

(b) $\varphi_{m}^{g}(u)-\varphi_{\propto}^{g}(u)>0, \psi_{n}^{g}(u)-\psi_{\infty}^{g}(u)<0$ for all $u \in[-a, a]$.

(c) There exists $1 \leq C<\infty$ such that

(i) $\frac{1}{C} \beta_{g}^{-m}\left|\varphi_{n}^{g}(u)-\varphi_{\infty}^{g}(u)\right| \leq\left|\varphi_{n+m}^{g}(u)-\varphi_{\infty}^{g}(u)\right| \leq C \beta_{g}^{-m}\left|\varphi_{n}^{g}(u)-\varphi_{\infty}^{g}(u)\right|$

$$
\frac{1}{C} \alpha_{g}^{m}\left|\psi_{n}^{g}(u)-\psi_{\infty}^{g}(u)\right| \leq\left|\psi_{n+m}^{g}(u)-\psi_{\infty}^{g}(u)\right| \leq C \alpha_{g}^{m}\left|\psi_{n}^{g}(u)-\psi_{\infty}^{g}(u)\right|
$$

(ii)

$$
\begin{gathered}
\left|D\left(\varphi_{m}^{g}-\varphi_{\infty}^{g}\right)(u)\right| \leq C\left|\varphi_{m}^{g}(u)-\varphi_{\infty}^{g}(u)\right| \\
\left|D\left(\psi_{n}^{g}-\psi_{\infty}^{g}\right)(u)\right| \leq C\left|\psi_{n}^{g}(u)-\psi_{\infty}^{g}(u)\right|,
\end{gathered}
$$

where $\alpha_{g}$ is the contracting eigenvalue of $D g(p(g))$ and $\beta_{g}$ is the expanding eigenvalue of $D g(q(g))$.

(d) Each $U_{n}(g)$ (resp. $S_{m}(g)$ ) has at most $r-1$ points of tangencies with leaves of the foliation $\mathscr{F}_{q(g)}^{s}\left(\right.$ resp. $\left.\mathscr{F}_{p(g)}^{u}\right)$.

Conditions (a) and (d) follow from the continuity of the $r$-jet of the foliations $\mathscr{F}_{p(g)}^{u}$ and $\mathscr{F}_{q(g)}^{s}$; (b) follows from condition (4) of the definition of $\mathcal{M}$ in $\$ 1$; (c) follows from the differentiability of the foliations $\mathscr{F}_{p(g)}^{u}, \mathscr{F}_{q(g)}^{s}$ and its 1 -jet. We need some lemmas.

LEMMA 6.2. If $D \varphi_{n}^{g}(u)=D \psi_{m}^{g}(u)$ and $\varphi_{n}^{g}(u)=\psi_{m}^{g}(u)$ for some $u \in(-a, a)$ and $m, n \in \mathbb{N}$ then

$$
\left|D \psi_{\infty}^{g}(u)-D \varphi_{\infty}^{g}(u)\right| \leq 2 C\left|\psi_{\infty}^{g}(u)-\varphi_{\infty}^{g}(u)\right| .
$$

Proof. From (b) it follows that

$$
\begin{gathered}
0<\varphi_{n}^{g}(u)-\varphi_{\infty}^{g}(u)=\psi_{m}^{g}(u)-\varphi_{\infty}^{g}(u)<\psi_{\infty}^{g}(u)-\varphi_{\infty}^{g}(u), \\
\varphi_{\infty}^{g}(u)-\psi_{\infty}^{g}(u)<\varphi_{n}^{g}(u)-\psi_{\infty}^{g}(u)=\psi_{m}^{g}(u)-\psi_{\infty}^{g}(u)<0 .
\end{gathered}
$$

Hence (using (c(ii))):

$$
\left|D \varphi_{n}^{g}(u)-D \varphi_{\infty}^{g}(u)\right| \leq C\left|\varphi_{n}^{g}(u)-\varphi_{\infty}^{g}(u)\right|<C\left|\varphi_{\infty}^{g}(u)-\psi_{\infty}^{g}(u)\right| .
$$

Therefore using $D \varphi_{n}^{g}(u)=D \psi_{m}^{g}(u)$ and the inequalities above:

$$
\begin{aligned}
\left|D \varphi_{\infty}^{g}(u)-D \psi_{\infty}^{g}(u)\right| & <C\left|\varphi_{\infty}^{g}(u)-\psi_{\infty}^{g}(u)\right|+\left|D \psi_{m}^{g}(u)-D \psi_{\infty}^{g}(u)\right| \\
& \leq C\left|\varphi_{\infty}^{g}(u)-\psi_{\infty}^{g}(u)\right|+C\left|\psi_{m}^{g}(u)-\psi_{\infty}^{g}(u)\right| \\
& \leq 2 C\left|\varphi_{\infty}^{g}(u)-\psi_{\infty}^{g}(u)\right| .
\end{aligned}
$$

LEMMA 6.3. Let $J_{g}=\left\{u \in(-a, a) ; \varphi_{\infty}^{g}(u) \leq \psi_{\infty}^{g}(u)\right.$ and $\left|D \psi_{\infty}^{g}(u)-D \varphi_{\infty}^{g}(u)\right| \leq$ $\left.2 C\left|\psi_{\infty}^{g}(u)-\varphi_{\infty}^{g}(u)\right|\right\}$. If $\mathcal{N}$ is small enough then $J_{g}$ has at most 2 r connected components.

Proof. Consider the functions $\theta_{ \pm}^{g}:[-a, a] \rightarrow \mathbb{R}$,

$$
\theta_{ \pm}^{g}(u)=\left(D \psi_{\infty}^{g}(u)-D \varphi_{\infty}^{g}(u)\right) \pm 2 C\left(\psi_{\infty}^{g}(u)-\varphi_{\infty}^{g}(u)\right) \text {. }
$$

Since the map $g \rightarrow \theta_{ \pm}^{g}$ from $\mathcal{N}$ to $C^{r}([-a, a], \mathbb{R})$ is continuous and $\theta_{ \pm}^{f}(u)=$ $r u^{r-1} \pm 2 C u^{r}$ it follows that for $\mathcal{N}$ small enough the set

$$
\left\{u \in[-a, a] ;\left|D \psi_{\infty}^{g}(u)-D \varphi_{\infty}^{g}(u)\right|=2 C\left|\psi_{\infty}^{g}(u)-\varphi_{\infty}^{g}(u)\right|\right\}
$$


has at most $2 r$ points. This proves the lemma because the boundary of $J_{g}$ is contained in this set.

LEMMA 6.4. If $\mathcal{N}$ is small enough there exists an integer $m$ such that for each $g \in \mathcal{N}$ and each connected component $J_{g}^{i}$ of $J_{g}$ there are integers $m_{0}=m_{0}(g, i)$ and $n_{0}=n_{0}(g, i)$ such that

and

$$
\psi_{n_{0}-m}^{g}(u)<\varphi_{\infty}^{g}(u)<\psi_{n_{0}+m}^{g}(u)
$$

for every $u \in J_{g}^{i}$.

$$
\varphi_{m_{0}+m}^{g}(u)<\psi_{\infty}^{g}(u)<\varphi_{m_{0}-m}^{g}(u)
$$

Proof. Let $\tilde{\varphi}^{g}, \tilde{\psi}_{n}^{g}: J_{g} \rightarrow \mathbb{R}$ be the maps $\tilde{\varphi}^{g}(u)=-\log \left(\psi_{\infty}^{g}(u)-\varphi_{\infty}^{g}(u)\right)$ and $\tilde{\psi}_{n}^{g}(u)=$ $-\log \left(\psi_{\infty}^{g}(u)-\psi_{n}^{g}(u)\right)$. From the definition of $J_{g}$ and from property (c(ii)) one has $\left|D \tilde{\psi}_{n}^{g}(u)\right| \leq C$, and $\left|D \tilde{\varphi}^{g}(u)\right|<2 C$ for every $u \in J_{g}$. If we take $V$ and $\mathcal{N}$ small enough so that $a<1 /(2 C)$ we have that $\left|\tilde{\varphi}^{g}\left(u_{1}\right)-\tilde{\varphi}^{g}\left(u_{2}\right)\right|<1$ and $\left|\psi_{n}^{g}\left(u_{1}\right)-\psi_{n}^{g}\left(u_{2}\right)\right|<\frac{1}{2}$, if $u_{1}, u_{2} \in J_{g}^{i}$. Moreover, from (c(i)), $\left|\tilde{\psi}_{n+m}(u)-\tilde{\psi}_{n}(u)\right|>2$ for every $n \in \mathbb{N}$ and $u \in J_{g}$ if $m$ is such that $-m \log \alpha_{g}-\log C>2$. Hence, if there exist $n_{0} \in \mathbb{N}$ and $u_{0} \in J_{g}^{i}$ such that $\tilde{\psi}_{n_{0}}^{g}\left(u_{0}\right)=\tilde{\varphi}^{g}\left(u_{0}\right)$ then

$$
\begin{aligned}
\tilde{\psi}_{n_{0}-m}(u) & <-1+\tilde{\psi}_{n_{0}}^{g}\left(u_{0}\right)=-1+\tilde{\varphi}^{g}\left(u_{0}\right) \\
& \leq \tilde{\varphi}^{g}(u) \leq 1+\tilde{\varphi}_{\mathrm{g}}\left(u_{0}\right) \\
& =1+\tilde{\psi}_{n_{0}}^{g}\left(u_{0}\right)<\tilde{\psi}_{n_{0}+m}(u)
\end{aligned}
$$

for every $u \in J_{\mathrm{g}}^{i}$ If this is not the case then there is an integer $n_{0}$ such that

$$
\tilde{\psi}_{n_{0}-m}^{g}(u)<\tilde{\psi}_{n_{0}}^{g}(u)<\tilde{\varphi}^{g}(u)<\tilde{\psi}_{n_{0}+1}^{g}(u)<\tilde{\psi}_{n_{0}+m}^{g}(u) \quad \text { for } u \in J_{g}^{i}
$$

This proves the first inequality of the lemma. The proof of the second one is similar.

LEMMA 6.5. Let $J_{g}^{i}$ be a connected component of $J_{g}$ and $n_{0}=n_{0}(g, i)$ be an integer such that $\psi_{n_{0}-m}^{g}(u)<\varphi_{\infty}^{g}(u)<\psi_{n_{0}+m}^{g}(u)$ for every $u \in J_{g}^{i}$. If $1-C^{2} \alpha_{g}^{m}>0$ then

$$
\#\left\{n \in \mathbb{N} ; \exists u \in J_{g}^{i}, \varphi_{n}^{g}(u) \in\left[\psi_{n_{0}+2 m}^{g}(u), \psi_{\infty}^{g}(u)\right]\right\}<\frac{-\log \left(\frac{1}{C^{3}} \alpha_{g}^{2 m}\left(1-C^{2} \alpha_{g}^{m}\right)\right)}{\log \beta_{g}} .
$$

Proof. Since $-\psi_{n_{0}-m}^{g}(u)>-\varphi_{\infty}^{g}(u)>-\psi_{n_{0}+m}^{g}(u)$ we have that

$$
\begin{aligned}
\log & \left(\psi_{\infty}^{g}(u)-\varphi_{\infty}^{g}(u)\right)-\log \left(\psi_{n_{0}+2 m}^{g}(u)-\varphi_{\infty}^{g}(u)\right) \\
& \leq \log \left(\psi_{\infty}^{g}(u)-\psi_{n_{0}-m}^{g}(u)\right)-\log \left(\psi_{n_{0}+2 m}^{g}(u)-\psi_{n_{0}+m}^{g}(u)\right) \\
& \leq-\log \left(\frac{1}{C^{2}} \alpha_{g}^{2 m}\left(1-C^{2} \alpha_{g}^{m}\right)\right) .
\end{aligned}
$$

Let $m_{1} \in \mathbb{N}$ be such that $\varphi_{m_{1}}^{g}(u)<\psi_{\infty}^{g}(u) \leq \varphi_{m_{1}-1}^{g}(u)$. If $k \in \mathbb{N}$ is such that $\psi_{\infty}^{g}(u)>$ 


$$
\begin{aligned}
\varphi_{m_{1}+k}^{g}(u)>\psi_{n_{0}+2 m}^{g}(u) \text { then } & \\
-\log \left(\frac{1}{C^{2}} \alpha_{g}^{2 m}\left(1-C^{2} \alpha_{g}^{m}\right)\right) & \geq \log \left(\psi_{\infty}^{g}(u)-\varphi_{\infty}^{g}(u)\right)-\log \left(\psi_{n_{0}+2 m}^{g}(u)-\varphi_{\infty}^{g}(u)\right) \\
& \geq \log \left(\varphi_{m_{1}}^{g}(u)-\varphi_{\infty}^{g}(u)\right)-\log \left(\varphi_{m_{1}+k}^{g}(u)-\varphi_{\infty}^{g}(u)\right) \\
& \geq-\log C+k \log \beta_{g} .
\end{aligned}
$$

Hence

$$
k \leq \frac{-\log \left(\frac{1}{C^{3}} \alpha_{g}^{2 m}\left(1-C^{2} \alpha_{g}^{m}\right)\right)}{\log \beta_{g}}
$$

This proves the lemma.

LEMMA 6.6. Let $J_{g}^{i}$ be a connected component of $J_{g}$. The cardinality of the set $\{n \in \mathbb{N}$; the graph of $\varphi_{n} \mid J_{g}^{i}$ has a tangency with the graph of $\psi_{k} \mid J_{\mathrm{g}}^{i}$ for some $\left.k\right\}$ is at most

$$
(r-1)\left(5 m+\frac{-\log \left(\frac{1}{C^{3}} \alpha_{g}^{2 m}\left(1-C^{2} \alpha_{g}^{m}\right)\right)}{\log \beta_{g}}\right) .
$$

Proof. Let $u_{j}^{k} \in J_{g}^{i}, j=1, \ldots, l$, be such that $\left(u_{j}^{k}, \psi_{k}^{g}\left(u_{j}^{k}\right)\right)$ are all the points where the graph of $\psi_{k}^{g} \mid J_{i}^{g}$ is tangent to the foliation $\mathscr{F}_{q(g)}^{s}$. Clearly $l \leq r-1$. If $n>m_{0}+m$ is such that the graph of $\varphi_{n}^{g} \mid J_{g}^{i}$ is tangent to the graph of $\psi_{k}^{g}$ for some $k \geq n_{0}+2 m$ then

$$
\varphi_{n}^{g}\left(u_{j}^{k}\right) \in\left[\psi_{n_{0}+2 m}^{g}\left(u_{j}^{k}\right), \varphi_{m_{0}+m}^{g}\left(u_{j}^{k}\right)\right] \subset\left[\psi_{n_{0}+2 m}^{g}\left(u_{j}^{k}\right), \psi_{\infty}^{g}\left(u_{j}^{k}\right)\right]
$$

for some $j=1, \ldots, l$. By lemma 6.5 there are at most

$$
\frac{-\log \left(\frac{1}{C^{3}} \alpha_{g}^{2 m}\left(1-C^{2} \alpha_{g}^{m}\right)\right)}{\log \beta_{g}}
$$

such integers. To finish the proof we notice that for each $k$ there are at most $l \leq r-1$ integers $n$ such that the graph of $\varphi_{n}^{g}$ has a tangency with the graph of $\psi_{k}^{g}$ and that $\varphi_{n}^{g}(u)>\psi_{k}^{g}(u)$ for every $u \in J_{g}^{i}$ if $k \leq n_{0}-m$ and $\varphi_{n}^{g}(u)>\psi_{l}^{g}(u)$ for every $u \in J_{g}^{i}$ if $n \leq m_{0}-m$. A simple combinatorial argument finishes the proof of the Lemma.

End of the proof of theorem 6.1. If the graph of $\varphi_{n}^{g}$ has a tangency with the graph of $\psi_{k}^{g}$ then, by lemma 6.2, there exists an integer $i$ such that the graph of $\varphi_{n}^{g} \mid J_{g}^{i}$ has a tangency with the graph of $\psi_{k}^{g}$. Since $J_{g}$ has at most $2 r$ connected components we have that the number of integers $n$ such that the graph of $\varphi_{n}^{g}$ is tangent to the graph of $\psi_{k}^{g}$ for some $k \in \mathbb{N}$ is at most

$$
2 r \cdot(r-1) \cdot\left(5 m+\frac{-\log \left(\frac{1}{C^{3}} \alpha_{g}^{2 m}\left(1-C^{2} \alpha_{g}^{m}\right)\right)}{\log \beta_{g}}\right) .
$$

The graph of $\varphi_{n}^{g}$ has at most $r-1$ points of tangency with the foliation $\mathscr{F}_{p(g)}^{u}$. Hence the total number of tangencies is at most

$$
(r-1) \cdot 2 r \cdot(r-1) \cdot\left(5 m+\frac{-\log \left(\frac{1}{C^{3}} \alpha_{g}^{2 m}\left(1-C^{2} \alpha_{g}^{m}\right)\right)}{\log \beta_{g}}\right)
$$

and this is clearly bounded by some $K>0$ in a small neighbourhood of $f$. 


\section{Appendix: Smoothness of Invariant Foliations}

In this appendix we will prove that certain invariant foliations are highly differentiable in some sense. More specifically, let $f$ be a $C^{\infty}$ diffeomorphism on a neighbourhood of $p$ in $\mathbb{R}^{n}$, with $f(p)=p$ and $p$ a hyperbolic saddle-point. Take a point $x \in W^{s}(p)$, a $C^{\infty} \operatorname{disc} \Sigma$ at $x$ transversal to $W^{s}(p)\left(\right.$ with $\left.\operatorname{dim} \Sigma=\operatorname{dim} W^{u}(p)\right)$ and let $V$ be a compact neighbourhood of $p$. According to the $\lambda$-Lemma the discs $f^{k}(\Sigma) \cap V$ converge in the $C^{r}$ sense to $W^{u}(p) \cap V$ as $k \rightarrow \infty$. Here $r \in \mathbb{N}$. For a proof of the $\lambda$-Lemma see [P1], [MP].

In this way one can construct invariant foliations. Fill a strip $N$ between $\Sigma$ and $f(\Sigma)$ with $C^{r}$-discs which are all transversal to $W^{s}(p)$. Iterating this foliation on $N$, one obtains an invariant unstable foliation $\mathscr{F}$ on $V$. According to the $\lambda$-Lemma the $r$-jet along leaves varies continuously, (we shall make this statement more precise below). But in many applications this is not sufficient. One needs to have that the $r$-jet along leaves varies 'in a $C^{1}$ sense'.

In this appendix we want to extend the $\lambda$-Lemma in two ways.

(a) Let $\mu_{1}, \ldots, \mu_{u}\left(\lambda_{1}, \ldots, \lambda_{s}\right)$ be the expanding (contracting) eigenvalues of $D f(p)$ and order these eigenvalues as follows:

$$
\left|\mu_{u}\right| \geq \cdots \geq\left|\mu_{1}\right|>1>\left|\lambda_{1}\right| \geq \cdots \geq\left|\lambda_{s}\right| .
$$

Assume that

$$
\left|\lambda_{1}\right|<\left|\mu_{1}\right| \cdot\left|\lambda_{s}\right| \text {. }
$$

Then the $r$-jet varies $C^{1}$ along leaves of $\mathscr{F}$, see theorem 7.3. Here $r \in \mathbb{N}$ is arbitrary. For $r=1$ this result is already contained in [HP, theorem (6.3)].

(b) Even if the disc $\Sigma$ has a tangency with $W^{s}(p)$ of polynomial type the result from above still holds under appropriate conditions, see theorem 7.4 below.

Clearly if $\left|\lambda_{1}\right|=\left|\lambda_{s}\right|$ (i.e. all contracting eigenvalues of $D f(p)$ have the same norm) then the condition $\left|\lambda_{1}\right|<\left|\mu_{1}\right| \cdot\left|\lambda_{s}\right|$ is automatically satisfied. In particular if $W^{u}(p)$ has codimension 1 then this condition can be dropped. From this one easily deduces that one can find $C^{1}$ linearising coordinates near a hyperbolic saddle-point $p$ if we are in the two-dimensional case. This result is not new, see [Ha]. The additional smoothness we obtain here is new and turns out to be essential in the estimates in this paper.

7.1. A fiber-contraction on a jet-bundle. Take a neighbourhood $V$ of $p$. Using local coordinates we can assume that $V$ is of the form $V=E_{1}(r) \times E_{2}(r)$, where $E_{1}(r)$

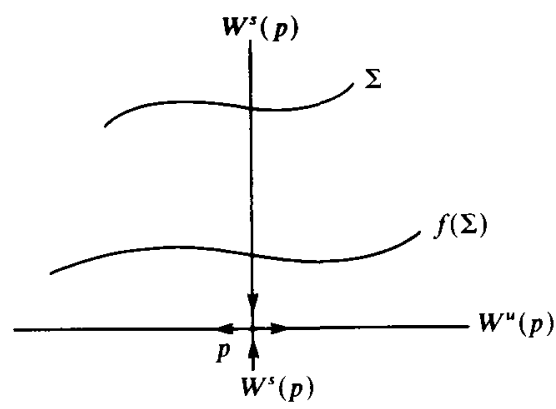

FIGURE 7.1 
$\left(E_{2}(r)\right)$ is a $u(s)$-dimensional ball with radius $r$. Assume $W^{u}(p) \cap V=E_{1}(r)$ and $W^{s}(p) \cap V=E_{2}(r)$.

Now let $\mathscr{E}^{k}\left(x_{0}, y_{0}\right)$ be the space of germs of $C^{k}$ functions $\left(E_{1}, x_{0}\right) \rightarrow\left(E_{2}, y_{0}\right)$. We say that $g_{1}, g_{2} \in \mathscr{E}^{k}\left(x_{0}, y_{0}\right)$ are $k$-equivalent, i.e. $g_{1} \sim_{k} g_{2}$ if the Taylor jet of $g_{1}$ and $g_{2}$ at $x_{0}$ agree up to $k$-th order (i.e. if $j^{k} g_{1}\left(x_{0}\right)=j^{k} g_{2}\left(x_{0}\right)$ ). Now define

and

$$
J^{k}\left(x_{0}, y_{0}\right)=\mathscr{E}^{k}\left(x_{0}, y_{0}\right) / \sim
$$

$$
J^{k}(V)=\bigcup_{\left(x_{0}, y_{0}\right) \in V} J^{k}\left(x_{0}, y_{0}\right)
$$

$J^{k}(V)$ is a smooth manifold. In fact one has the natural identification $D_{k}: J^{k}(V) \rightarrow$ $V \times \mathbb{R}^{l(1)} \times \cdots \times \mathbb{R}^{l(k)}$ which identifies the $k$-jet with the coefficients in the Taylor expansion. Here $l(j)$ is the dimension of the space of homogeneous polynomials from $\mathbb{R}^{u}$ to $\mathbb{R}^{s}$ of degree $j$. Similarly one has the map $\pi_{k, k-1}: J^{k}(V) \rightarrow J^{k-1}(V)$ which maps the $k$-jet of a function to its $(k-1)$-jet. In this way one gets the commutative diagram

$$
\begin{array}{ccc}
J^{k}(V) \stackrel{D_{k}}{\simeq} & V \times \mathbb{R}^{l(1)} \times \cdots \times \mathbb{R}^{l(k)} \\
\downarrow \pi_{k, k-1} & \downarrow \tilde{\pi}_{k, k-1} \\
\vdots & \vdots \\
J^{2}(V) \stackrel{D_{2}}{\longrightarrow} & V \times \mathbb{R}^{l(1)} \times \mathbb{R}^{l(2)} \\
\downarrow \pi_{2,1} & & \downarrow \tilde{\pi}_{2,1} \\
J^{1}(V) \stackrel{D_{1}}{\longrightarrow} & V \times \mathbb{R}^{l(1)} \\
\downarrow \pi_{1} & & \downarrow \tilde{\pi}_{1} \\
V & \stackrel{\text { id }}{\longrightarrow} & V .
\end{array}
$$

Here $\quad \tilde{\pi}_{j, j-1}: V \times \mathbb{R}^{l(1)} \times \cdots \times \mathbb{R}^{l(j)} \rightarrow V \times \mathbb{R}^{l(1)} \times \cdots \times \mathbb{R}^{l(j-1)}$ is the projection $\left(x,\left(v_{1}, \ldots, v_{j}\right)\right) \rightarrow\left(x,\left(v_{1}, \ldots, v_{j-1}\right)\right)$. It will also be useful to work with jet spaces of functions with bounded derivatives. So let $B^{j}=\left\{v \in \mathbb{R}^{l(1)} \times \cdots \times \mathbb{R}^{l(j)} ; \quad v=\right.$ $\left(v_{1}, \ldots, v_{j}\right), v_{i} \in \mathbb{R}^{l(i)}$ and $\left.\left|v_{i}\right| \leq 1, i=1,2, \ldots, j\right\}$. Define $X^{j}(V)=D_{j}^{-1}\left(V \times B^{j}\right)$. As before we have a commutative diagram.

Now assume that $f: V \rightarrow \mathbb{R}^{m}$ is a diffeomorphism with 0 as a hyperbolic fixed point. Let $\lambda_{1}, \ldots, \lambda_{s}\left(\mu_{1}, \ldots, \mu_{u}\right)$ be the contracting (expanding) eigenvalues of $D f(0)$. Assume that they are ordered as follows:

$$
\left|\mu_{u}\right| \geq \cdots \geq\left|\mu_{1}\right|>1>\left|\lambda_{1}\right| \geq \cdots \geq\left|\lambda_{s}\right| \text {. }
$$

For $x \in V=E_{1}(r) \times E_{2}(r)$ one can write $D f(x), D f^{-1}(x): E_{1} \times E_{2} \rightarrow E_{1} \times E_{2}$ as follows:

$$
D f(x)=\left(\begin{array}{ll}
A(x) & B(x) \\
C(x) & D(x)
\end{array}\right), \quad D f^{-1}(x)=\left(\begin{array}{cc}
\tilde{A}(x) & \tilde{B}(x) \\
\tilde{C}(x) & \tilde{D}(x)
\end{array}\right) .
$$

Since $W^{u}(p) \cap V=E_{1}(r)$ and $W^{s}(p) \cap V=E_{2}(r)$ the matrices $B(x), \tilde{B}(x), C(x)$ and $\tilde{C}(x)$ vanish for $x=0$. Moreover for each $\delta>0$ one can choose $r>0$ so small 
that for $x \in V$,

$$
\begin{aligned}
& \|A(x)\| \leq\left|\mu_{u}\right|+\delta, \quad\|B(x)\| \leq \delta, \\
& \|C(x)\| \leq \delta, \quad\|D(x)\| \leq\left|\lambda_{1}\right|+\delta .
\end{aligned}
$$

Similarly

$$
\begin{aligned}
& \|\tilde{A}(x)\| \leq\left(\left|\mu_{1}\right|-\delta\right)^{-1}, \quad\|\tilde{B}(x)\| \leq \delta, \\
& \|\tilde{C}(x)\| \leq \delta, \quad\|\tilde{D}(x)\| \leq\left(\left|\lambda_{s}\right|-\delta\right)^{-1} .
\end{aligned}
$$

Furthermore take a neighbourhood $U$ of $0, U \subset V$, such that $f(U) \subset V$. In other words if $\left(x_{0}, y_{0}\right) \in U$, then $f\left(x_{0}, y_{0}\right)=\left(f^{1}\left(x_{0}, y_{0}\right), f^{2}\left(x_{0}, y_{0}\right)\right)=\left(x_{1}, y_{1}\right) \in V$. Furthermore take $\mathscr{E}_{1}^{k}\left(x_{0}, y_{0}\right)=\left\{g \in \mathscr{E}^{k}\left(x_{0}, y_{0}\right):\left|D g\left(x_{0}\right)\right|<1\right\}$. Suppose $f$ is $C^{n}$. Then define for $k \leq n$,

$$
\Gamma_{\left(x_{0}, y_{0}\right)}: \mathscr{E}^{k}\left(x_{0}, y_{0}\right) \rightarrow \mathscr{E}^{k}\left(x_{1}, y_{1}\right)
$$

by defining $\Gamma_{\left(x_{0}, y_{0}\right)}(g):\left(E_{1}, x_{1}\right) \rightarrow\left(E_{2}, y_{1}\right)$ to be the germ of the function

$$
t \rightarrow\left(f^{2}(\mathrm{id}, g)\right) \circ\left(f^{1}(\mathrm{id}, g)\right)^{-1}(t),
$$

see figure 7.2 .

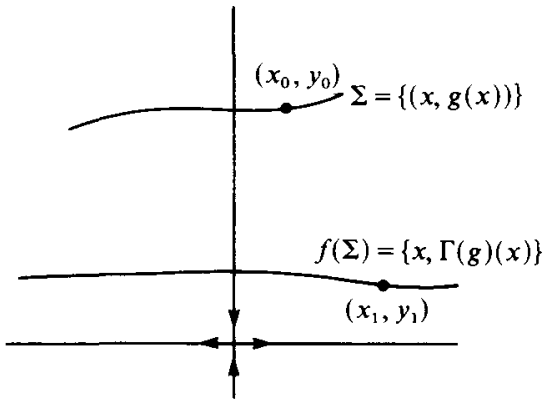

Figure 7.2

Let $V$ be so small that $\|A(x)\|>\|B(x)\|$, for all $x$ in $V$. Since for $g \in$ $\mathscr{E}^{k}\left(x_{0}, y_{0}\right),\left|D g\left(x_{0}\right)\right|<1$, the map $t \rightarrow f^{1} \circ(\mathrm{id}, g)(t)$ is invertible at $t=x_{0}$, for $\left(x_{0}, y_{0}\right)$ in $V$. Hence $\Gamma_{\left(x_{0}, y_{0}\right)}$ is a well defined map for $\left(x_{0}, y_{0}\right)$ in $V$. (This also follows from the $\lambda$-Lemma). Clearly $\Gamma$ induces a map on the $k$-jet level: This map $\Gamma_{k}: X^{k}(U) \rightarrow$ $J^{k}(V)$ is defined by

$$
\Gamma_{k}\left(j^{k} g\left(x_{0}\right)\right)=J^{k}\left(\Gamma_{\left(x_{0}, y_{0}\right)} g\right)\left(x_{1}\right) .
$$

Presently we will show that the map $\Gamma_{k}$ contracts the fibers of $\pi_{k, k-1}: J^{k} \rightarrow J^{k-1}$. So define for $g \in \mathscr{E}^{k}\left(x_{0}, y_{0}\right)$,

$$
|g|_{k}=\max _{1 \leq i \leq k}\left|D g^{i}\left(x_{0}\right)\right| \text {. }
$$

LEMMA 7.1. Let $k \leq n$ and $\varepsilon>0$ be given. Then we can choose $V$ so small that for any $g_{1}, g_{2} \in J^{k}(V)$ with $\pi_{k, k-1}\left(g_{1}\right)=\pi_{k, k-1}\left(g_{2}\right)$ one has

(a) $\left|\Gamma_{k}\left(g_{1}\right)-\Gamma_{k}\left(g_{2}\right)\right| \leq\left(\left(\left|\lambda_{1}\right| /\left|\mu_{1}\right|^{k}\right)+\varepsilon\right) \cdot\left|g_{1}-g_{2}\right|_{k}$;

(b) $\left.D\left(f^{-1} \mid V\right)\right) \leq\left(1 /\left|\lambda_{s}\right|\right)+\varepsilon$.

Proof. Statement (b) is obvious from equations (7.2) so let us prove (a). Here we 
are going to use the following formulae: if $\phi, \psi_{1}, \psi_{2}$ are $C^{k}$ and $\left|\psi_{1}-\psi_{2}\right|_{k-1}=0$ then

$$
\begin{aligned}
& \left|\phi \circ \psi_{1}-\phi \circ \psi_{2}\right|_{k}=|\phi|_{1} \cdot\left|\psi_{1}-\psi_{2}\right|_{k}, \\
& \left|\psi_{1} \circ \phi-\psi_{2} \circ \phi\right|_{k}=\left|\psi_{1}-\psi_{2}\right|_{k} \cdot\left(|\phi|_{1}\right)^{k} . \\
& \left|\left(\psi_{1}\right)^{-1}-\left(\psi_{2}\right)^{-1}\right|_{k} \leq\left|\psi_{1}-\psi_{2}\right|_{k} \cdot\left(\left|\psi_{1}^{-1}\right|_{1}\right)^{k+1} .
\end{aligned}
$$

Now by assumption the $(k-1)$-jets of $\Gamma^{k} g_{1}$ and $\Gamma^{k} g_{2}$ are equal. Therefore $\left|\Gamma^{k} g_{1}-\Gamma^{k} g_{2}\right|_{k}$ can be estimated (using the equalities from above) by:

$$
\begin{aligned}
& \mid\left. f^{2} \circ(\text { id, } g)\right|_{1} \cdot \mid\left(f^{1} \circ\left(\text { id, } g_{1}\right)\right)^{-1}-\left.\left(f^{1} \circ\left(\text { id, } g_{2}\right)\right)^{-1}\right|_{k} \\
& \quad+\mid f^{2} \circ\left(\text { id, } g_{1}\right)-\left.f^{2} \circ\left(\text { id, } g_{2}\right)\right|_{k} \cdot\left\{\mid\left.\left(f^{1} \circ(\text { id, } g)\right)^{-1}\right|_{1}\right\}^{k} .
\end{aligned}
$$

Here we can take for $g$ either $g_{1}$ or $g_{2}$. For $V$ sufficiently small this is majorised by (see equations $(7.1),(7.2))$.

$$
\frac{\left(\left|\lambda_{1}\right|+\delta\right)}{\left(\left|\mu_{1}\right|-\delta\right)^{k+1}} \cdot \delta \cdot\left|g_{1}-g_{2}\right|_{k}+\frac{\left(\left|\lambda_{1}\right|+\delta\right)}{\left(\left|\mu_{1}\right|-\delta\right)^{k}} \cdot\left|g_{1}-g_{2}\right|_{k}
$$

So for $V$ sufficiently small, inequality (a) holds.

Corollary. $\Gamma_{k}\left(X^{k}(U)\right) \subset X^{k}(V)$.

7.2. Differentiability of invariant foliations; a differentiable version of the $\lambda$-Lemma. As before let $V=E_{1}(r) \times E_{2}(r)$ and $f$ be a $C^{n}$ diffeomorphism with a saddle-point at 0 . Let $U$ be a neighbourhood of 0 so that $\Gamma_{k}\left(X^{k}(U)\right) \subset X^{k}(V)$ (and in particular $f(U) \subset V)$. Such a neighbourhood exists, see the corollary above.

Let $\mathscr{F}$ be some foliation on $U$, not necessarily invariant, whose leaves are graphs of $C^{n}$ functions $E_{1}(r) \rightarrow E_{2}(r)$. For $k \leq n$, this defines a section $\sigma_{k}: U \rightarrow J^{k}(U)$ where $\sigma_{k}\left(x_{1}, y_{1}\right)$ is the $k$-jet at $x_{1} \in E_{1}(r)$ of the function whose graph is the leaf of $\mathscr{F}$ through the point $\left(x_{1}, y_{1}\right) \in U$. Suppose that the foliation $\mathscr{F}$ is chosen so that $\sigma_{k}(U) \subset X^{k}(U)$. In particular this implies that the leaves of $\mathscr{F}$ are transversal to $W^{s}(p)$. This foliation $\mathscr{F}$ is invariant precisely when the following diagram commutes:

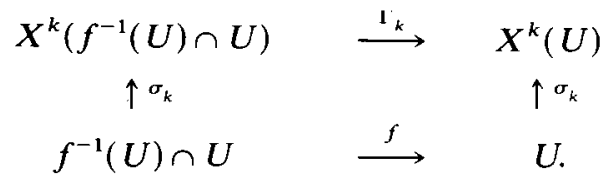

Let us now show how to construct such invariant foliations. In order to do this take a foliation $\mathscr{F}$ so that for each leaf $\Sigma$ in $U-f(U)$ the image $f(\Sigma) \cap U$ is also a leaf of $\mathscr{F}$. We claim that we can find a new invariant foliation $\mathscr{F}^{*}$ which coincides with $\mathscr{F}$ on $U-f(U)$. In fact:

LEMMA 7.2. Let $\mathscr{F}$ be as above and such that for the corresponding section $\sigma_{k}$ one has $\sigma_{k}(U) \subset X^{k}(U), k \leq n$. Then there exists an invariant foliation $\mathscr{F}^{*}$ which coincides with $\mathscr{F}$ on $U-f(U)$. The sections $\sigma_{k}^{*}: U \rightarrow X^{k}(U)$ corresponding to $\mathscr{F}^{*}$ are $C^{0}$ for $k \leq n$.

Proof. Follows from the $\lambda$-Lemma [P1].

Remark that if we assume that $\sigma_{n-1}: U \rightarrow X^{n-1}(U)$ is $C^{1}$ on $U-f(U)$, then the restriction of $\sigma_{n-1}^{*}: U \rightarrow X^{n-1}(U)$ is also $C^{1}$ on $U-W^{u}(0)$. Let us show that in fact $\sigma_{n-1}^{*}: U \rightarrow X^{n-1}(U)$ is $C^{1}$ on $U$. 
THEOREM 7.3 ( $C^{1}$-version of the $\lambda$-Lemma). Let $\mathscr{F}^{*}$ be an invariant foliation inducing a continuous section $\sigma_{n}^{*}: U \rightarrow X^{n}(U)$ so that the restriction of $\sigma_{n-1}^{*}: U \rightarrow X^{n-1}(U)$ to $U-W^{u}(0)$ is $C^{1}$. Then $\sigma_{n-1}^{*}: U \rightarrow X^{n-1}(U)$ is $C^{1}$, provided $\left|\lambda_{1}\right|<\left|\mu_{1}\right| \cdot\left|\lambda_{s}\right|$.

Proof. Let $\mathscr{G}^{k}$ be the space of sections $\sigma_{k}: U \rightarrow J^{k}(U)$ such that $\sigma^{k}$ restricted to $U-f(U)$ coincides with $\sigma_{k}^{*}$ (the section corresponding to $\mathscr{F}^{*}$ ). Endow this space with the supremum norm $\|\sigma\|_{k}=\sup \left\{|\sigma(x)|_{k}, x \in U\right\} . \mathscr{G}^{k}$ is a complete metric space. Let $\mathscr{G}^{k}(1)$ be the unit ball in $\mathscr{G}^{k}$.

Using the graph transformation $\Gamma_{k}$ we define $\phi_{k}: \mathscr{G}^{k}(1) \rightarrow \mathscr{G}^{k}(1)$ as follows. Let $\sigma \in \mathscr{G}^{k}(1)$. Then

$$
\phi_{k}(\sigma)(x)= \begin{cases}\sigma_{k}^{*}(x) & \text { if } x \in U-f(U) \\ \Gamma_{k}\left(\sigma\left(f^{-1}(x)\right)\right) & \text { if } x \in U \cap f(U)\end{cases}
$$

Since we had chosen $U$ so that $\Gamma_{k}\left(X^{k}(U)\right) \subset X^{k}(V)$ it follows that $\phi_{k}$ is well defined. Remark that $\sigma \in \mathscr{G}^{k}(1)$ is an invariant section precisely when the diagram above commutes, i.e. when $\sigma=\Gamma_{k}\left(\sigma \circ f^{-1}\right)$. This is equivalent to $\phi_{k}(\sigma)=\sigma$. From the $\lambda$-Lemma, see lemma 7.2, it follows that $\phi_{k}$ has a unique attracting fixed point $\sigma$ (for $k \leq n$ ). We will prove that $\sigma$ is $C^{1}$ (for $k \leq n-1$ ) by induction on $k$.

The idea will be the following. Suppose we can show that for some $\sigma \in \mathscr{G}^{k}(1)$ one has that $D\left(\left(\phi_{k}\right)^{m}(\sigma)\right)$ converges as $m \rightarrow \infty$. (Here $\left(\phi_{k}\right)^{m}$ is the $m$ th iterate of $\phi_{k}$.) Since $\left(\phi_{k}\right)^{m}(\sigma)$ converges to the fixed point $\sigma_{k}^{*}$ it follows that $\sigma_{k}^{*}$ is $C^{1}$. So it suffices to show that $D\left(\left(\phi_{k}\right)^{m}(\sigma)\right)$ converges as $m \rightarrow \infty$. We will do this using the fibre contraction theorem.

We start by noting that $J^{k}(U)$ is homeomorphic to $U \times \mathrm{Fib}^{1} \times \cdots \times \mathrm{Fib}^{k}$, where $U \simeq \mathbb{R}^{n}, \mathrm{Fib}^{(i)} \simeq \mathbb{R}^{l(i)}$. Let $p_{i}: J^{k}(U) \rightarrow \mathrm{Fib}^{(i)}$ be the projection on the $i$ th fiber. Now define $\mathscr{H}^{k}$ to be the space of continuous maps $H: U \times \mathbb{R}^{m} \rightarrow \mathrm{Fib}^{k}$ with $H(x, v)=$ $H_{x}(v)$, where $H_{x}: \mathbb{R}^{m} \rightarrow \mathrm{Fib}^{k}$ is linear and $H_{x}$ coincides with the derivative of $p_{k} \circ \sigma_{k}^{*}$ at $x$ for $x$ in $U-f(U)$. Endow $\mathscr{H}^{k}$ with the norm $\|H\|=\sup _{x \in U}\left\|H_{x}\right\|$.

Let us now prove the first induction step that $\sigma_{1}^{*}$ is $C^{1}$. In order to do this fix $\sigma \in \mathscr{G}^{1}(1)$. For this $\sigma$ we define a transformation $\psi_{1, \sigma}: \mathscr{H}^{1} \rightarrow \mathscr{H}^{1}$ by

$$
\left(\psi_{1, \sigma} H\right)_{x}(v)= \begin{cases}D\left(p_{1} \circ \sigma_{1}^{*}\right)_{x}(v) & \text { if } x \in U-f(U) \\ D\left(p_{1} \circ \Gamma_{1}\right)_{\sigma(y)}\left(w, H_{y}(w)\right) & \text { if } x \in U \cap f(U) .\end{cases}
$$

Here $w=\left(d f_{x}\right)^{-1}(v)$ and $y=f^{-1}(x)$. Remark that for $x \in U \cap f(U)$ one has $y=$ $f^{-1}(x) \in U \cap f^{-1}(U)$ and hence $\left(w, H_{y}(w)\right) \in T_{\sigma(y)} X^{1}\left(U \cap f^{-1}(U)\right)$. Therefore for $x \in U \cap f(U),\left(\psi_{1, \sigma} H\right)_{x}(v)$ is the derivative of

$$
p_{1} \circ \Gamma_{1}: X^{1}\left(U \cap f^{-1}(U)\right) \rightarrow \mathrm{Fib}^{1}
$$

at $\sigma(y)$ in the direction $\left(w, H_{y}(w)\right)$, and therefore $\psi_{1, \sigma}(D \sigma)=D\left(\phi_{1}^{\circ} \sigma\right)$ if $\sigma$ is $C^{1}$. Let us show that $\psi_{1, \sigma}$ is a contraction. Indeed take $H, K \in \mathscr{H}^{1}$. Then

$$
\left(\psi_{1, \sigma} H-\psi_{1, \sigma} K\right)_{x}(v)= \begin{cases}0 & \text { for } x \in U-f(U) \\ D\left(p_{1} \circ \Gamma_{1}\right)_{\sigma(y)}\left(0,\left(H_{y}-K_{y}\right)(w)\right) & \text { otherwise, }\end{cases}
$$


where $w \doteq\left(D f_{x}\right)^{-1}(v)$. From lemma 7.1 it follows that

$$
\begin{aligned}
\left\|D\left(p_{1} \circ \Gamma_{1}\right)_{\sigma(y)}\left(0,\left(H_{y}-K_{y}\right)(w)\right)\right\| & \leq\left(\frac{\left|\lambda_{1}\right|}{\left|\mu_{1}\right|}+\varepsilon\right) \cdot\left\|\left(H_{y}-K_{y}\right)(w)\right\| \\
& \leq\left(\frac{\left|\lambda_{1}\right|}{\left|\mu_{1}\right|}+\varepsilon\right) \cdot\left\|H_{y}-K_{y}\right\| \cdot\left\|D f_{x}^{-1}(v)\right\| \\
& \leq\left(\frac{\left|\lambda_{1}\right|}{\left|\mu_{1}\right|}+\varepsilon\right) \cdot\left(\frac{1}{\left|\lambda_{s}\right|}+\varepsilon\right) \cdot\left\|H_{y}-K_{y}\right\| \cdot\|v\| .
\end{aligned}
$$

Hence

$$
\left\|\psi_{1, \sigma} H-\psi_{1, \sigma} K\right\| \leq \lambda \cdot\|H-K\|,
$$

where $\left.\lambda=\left(\left(\left|\lambda_{1}\right| /\left|\mu_{1}\right|\right)+\varepsilon\right) \cdot\left(1 /\left|\lambda_{s}\right|\right)+\varepsilon\right)$ can be chosen smaller than one, by taking $\varepsilon>0$ sufficiently small. Hence

$$
\left\|\psi_{1, \sigma} H-\psi_{1, \sigma} K\right\| \leq \lambda \cdot\|H-K\|,
$$

i.e. $\psi_{1, \sigma}$ is a contraction. Now we are in the position to prove that $\sigma_{1}^{*}$ is $C^{\prime}$. In fact let $\theta_{1}: \mathscr{G}^{1} \times \mathscr{H}^{1} \rightarrow \mathscr{G}^{1} \times \mathscr{H}^{1}$ be defined by

$$
\theta_{1}(\sigma, H)=\left(\phi_{1}(\sigma), \psi_{1, \sigma}(H)\right)
$$

Remark that $\sigma_{1}^{*}$ is the unique attracting fixed point of $\phi_{1}$ and since $\psi_{1, \sigma}$ is a contraction for every $\sigma$, it follows from the Fiber Contraction Theorem (see [HP]) that $\theta_{1}$ has a unique attracting fixed point $\left(\sigma_{1}^{*}, H_{1}^{*}\right)$. So let $\sigma$ be $C^{1}$. Then

$$
\theta_{1}(\sigma, D \sigma)=\left(\phi_{1}(\sigma), \psi_{1, \sigma}(D \sigma)\right)=\left(\phi_{1}(\sigma), D \phi_{1} \sigma\right)
$$

and therefore $\left(\theta_{1}\right)^{l}(\sigma, D \sigma)=\left(\phi_{1}^{l}(\sigma), D \phi_{1}^{l}(\sigma)\right)$. Hence $D\left(\phi_{1}^{l}\right)(\sigma)$ converges to $H_{1}^{*}$ and $\phi_{1}^{l}(\sigma)$ to $\sigma_{1}^{*}$ as $l \rightarrow \infty$. It follows that $\sigma_{1}^{*}$ is $C^{1}$ and $D \sigma_{1}^{*}=H_{1}^{*}$. This finishes the first induction step.

Let us now prove that $\sigma_{2}^{*}: U \rightarrow X^{2}(U)$ is $C^{1}$. Let $\sigma \in \mathscr{G}^{2}$ and $H_{1} \in \mathscr{H}^{1}$. Define $\psi_{2, \sigma, H_{1}}: \mathscr{H}^{2} \rightarrow \mathscr{H}^{2}$ by

$$
\psi_{2, \sigma, H_{1}}\left(H_{2}\right)=\left\{\begin{array}{l}
D\left(p_{2} \circ \sigma_{2}^{*}\right)_{x}(v) \quad \text { if } x \in U-f(U), \\
D\left(p_{2} \circ \Gamma_{2}\right)_{\sigma(y)}\left(w, H_{1, y}(w), H_{2, y}(w)\right)
\end{array} \quad \text { if } x \in U \cap f(U) .\right.
$$

Here $w=\left(D f_{x}\right)^{-1}(v)$ and $y=f^{-1}(x)$. As before take $H_{2}, K_{2} \in \mathscr{H}^{2}$. Then

$$
\left(\psi_{2, \sigma, H_{1}}\left(H_{2}\right)-\psi_{2, \sigma, H_{1}}\left(K_{2}\right)\right)_{x}(v)= \begin{cases}0 & \text { if } x \in U-f(U) \\ D\left(p_{2} \circ \Gamma_{2}\right)_{\sigma(y)}\left(0,0,\left(H_{2, y}-K_{2, y}\right)(w)\right) \text { otherwise, }\end{cases}
$$

where $y=f^{-1}(x)$ and $w=\left(D f_{x}\right)^{-1}(v)$. As before one deduces that $\psi_{2, \sigma, H_{1}}$ is a contraction, using lemma 7.1. Now define a map $\theta_{2}:\left(\mathscr{G}^{2} \times \mathscr{H}^{1}\right) \times \mathscr{H}^{2} \varsigma$ by

$$
\left(\left(\sigma, H_{1}\right), H_{2}\right) \mapsto\left(\left(\phi_{2}(\sigma), \psi_{1, \sigma}\left(H_{1}\right)\right), \psi_{2, \sigma, H_{1}}\left(H_{2}\right)\right) \text {. }
$$

As before the Fiber Contraction Theorem implies that $\theta_{2}$ has a unique attracting fixed point $\left(\sigma_{2}^{*}, H_{1}^{*}, H_{2}^{*}\right)$. If $\sigma \in \mathscr{G}^{2}$ is $C^{1}$, then

$$
\theta_{2}\left(\sigma, D\left(p_{1} \circ \sigma\right), D\left(p_{2} \circ \sigma\right)\right)=\left(\phi_{2}(\sigma), D\left(p_{1} \circ \phi_{2} \circ \sigma\right), D\left(p_{2} \circ \phi_{2} \circ \sigma\right)\right) \text {. }
$$

As before it follows that $D\left(p_{2} \circ \sigma_{2}^{*}\right)=H_{2}^{*}$. From the previous induction step $D\left(p_{1} \circ \sigma_{2}^{*}\right)=D\left(\sigma_{1}^{*}\right)=H_{1}^{*}$. Hence $\sigma_{2}^{*}$ is $C^{1}$. Similarly one proves by induction that the fixed point $\sigma_{k}^{1} \in \mathscr{G}^{k}$ of $\phi_{k}$ is $C^{1}$ for $k \leq n-1$. 
COROllary 1. Let $f: M \rightarrow M$ be a diffeomorphism with a saddle-point $p$ and let $\operatorname{codim}\left(W^{u}(p)\right)=1$. Then there exists an invariant unstable foliation $\mathscr{F}^{*}$ for $p$, such that the k-jet along leaves is a $C^{1}$ function.

Proof. This follows from theorem 7.3 because in this case the condition $\left|\lambda_{1}\right|<\left|\mu_{1}\right| \cdot\left|\lambda_{s}\right|$ is trivially satisfied.

Remark. Let $\mathscr{F}$ be an invariant foliation on $V-W^{u}(p)$, where $V$ is a neighbourhood of $W^{u}(p)$. If $\mathscr{F}$ satisfies the conclusion of corollary 1 , then the foliation $\mathscr{F} *=\mathscr{F} \cup$ $W^{u}(p)$ on $V$ also satisfies the conclusion of corollary 1 . In other words the foliation $\mathscr{F}^{*}$ can be found as the extension of a given one on $V-W^{u}(p)$.

COROLlaRY 2. If the diffeomorphism $f$ and the foliation $\mathscr{F}$ depend $C^{s}$ on parameters, then the corresponding invariant foliation $\mathscr{F}^{*}(f, \mathscr{F})$ also depends $C^{s}$ on this parameter.

Proof. This can be proved with the same methods as used in [HP] for showing that the unstable manifolds depend continuously on $f$.

7.3. $C^{1}$-linearisability near saddle-points in the two-dimensional case. Let $f: M \rightarrow M$ be a diffeomorphism with a hyperbolic saddle-point $p$. Assume $\operatorname{dim}(M)=2$. From corollary 1 of theorem 7.3 one can obtain invariant stable and unstable foliations for $p, \mathscr{F}^{s}$ and $\mathscr{F}^{u}$, which are $C^{1}$. From this one obtains a $C^{1}$ invariant projection $\pi_{s}\left(\pi_{u}\right)$ from a neighbourhood $V$ of $p$ onto $W^{s}(p)\left(W^{u}(p)\right)$ by projecting along the leaves of $\mathscr{F}^{u}\left(\mathscr{F}^{s}\right)$. By construction one has

$$
\pi_{s} \circ f=f \circ \pi_{s}, \quad \pi_{u} \circ f=f \circ \pi_{u} .
$$

Then take $C^{1}$ coordinates on $W^{s}(p)$ and on $W^{u}(p)$ so that $f \mid W^{s}(p)$ and $f \mid W^{u}(p)$ are linear with respect to these coordinates. (This is not hard to do, since $W^{s}(p)$ and $W^{u}(p)$ are 1-dimensional). Using these coordinates and $\pi_{s}, \pi_{u}$ one obtains a $C^{1}$-linearising coordinate system for $f$ near $p$. This result was already known, see [Ha] and [HP, theorem (6.1)]. The fact that there is additional smoothness i.e. that $\sigma_{n-1}^{*}: V \rightarrow X^{n-1}(V)$ is $C^{1}$, is new.

7.4. Differentiability of invariant foliations with polynomial tangencies. In $\$ 7.2$ we have extended foliations which were smooth and transversal to $W^{s}(p)$. In this section we will consider foliations with leaves which have a tangency of finite order with $W^{s}(p)$, see figure 7.3 .

More precisely take a neighbourhood $V$ of $p$. Using local coordinates we can assume that $V=E_{1}(r) \times E_{2}(r)$ where $E_{1}(r)$ (resp. $\left.E_{2}(r)\right)$ is a $u($ resp. $s)$-dimensional ball of

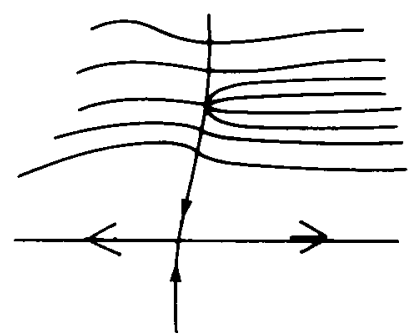

FIGURE 7.3 
radius $r$. Assume $W^{u}(p) \cap V=E_{1}(r), W^{s}(p) \cap V=E_{2}(r)$. As before define the space of germs $\mathscr{E}^{k}\left(x_{0}, y_{0}\right)$. For $g \in \mathscr{E}^{k}\left(x_{0}, y_{0}\right)$ and $0 \leq \delta<1$ define a new norm

$$
|g|_{k, \delta}=\max _{i=1,2, \ldots, k}\left\{\left|D^{i} g\left(x_{0}\right)\right| \cdot\left|x_{0}\right|^{i-\delta}\right\} \text {. }
$$

Furthermore let

$$
X^{k, \delta}(V)=\left\{g \in J^{k}(V) ;|g|_{k, \delta} \leq 1\right\} .
$$

(We could also introduce extra coefficients $a_{i}>0$ and consider $\left|D^{i} g\left(x_{0}\right)\right| \cdot\left|x_{0}\right|^{i-\delta} \cdot a_{i}$, but this would have no essential effect on the sequel.)

Now consider some foliation $\mathscr{F}^{u}$ on $V$ whose leaves are graphs of functions $E_{1}(r) \rightarrow E_{2}(r)$ which are $C^{k}$ on $E_{1}(r)-0$. This defines a section $\sigma_{k}: V-W^{s}(p) \rightarrow$ $J^{k}\left(V-W^{s}(p)\right)$ as before. We assume now that

$$
\sigma_{k}\left(V-W^{s}(p)\right) \subset X^{k, \delta}\left(V-W^{s}(p)\right),
$$

for some $\delta>0$. In other words we allow the foliation $\mathscr{F}^{u}$ to have tangencies with $W^{s}(p)$ of order at most $1 /(1-\delta)$. We have now the following generalization of the $\lambda$-Lemma and of theorem 7.3.

THEOREM 7.4. Assume that $\sigma_{k}$ is as above and that for the corresponding $\delta$ one has,

$$
\left|\frac{\lambda_{1}}{\lambda_{s}}\right| \cdot \frac{\left|\mu_{u}\right|^{k-\delta}}{\left|\mu_{1}\right|^{k}}<1 \text {. }
$$

(a) If $k \leq n$, where $n$ is the degree of differentiability of $f$, then $\mathscr{F}$ can be extended to an invariant foliation $\mathscr{F}^{*}$ on $V$ such that $\sigma: V-W^{s}(p) \rightarrow X^{k, \delta}(V)$ is continuous.

(b) If $\sigma: V-\left(W^{s}(p) \cup W^{u}(p)\right) \rightarrow X^{k, \delta}(V)$ is $C^{1}$, then in fact $\sigma: V-W^{s}(p) \rightarrow$ $X^{k, \delta}(V)$ is $C^{1}$.

Proof. One cannot deduce (a) from the $\lambda$-Lemma because $\mathscr{F}^{u}$ has tangencies with $W^{s}(p)$. So define as in theorem 7.3:

$$
\mathscr{G}^{k, \delta}=\left\{\sigma: V \backslash W^{s}(p) \rightarrow X^{k, \delta}(V), \sigma=\sigma^{*} \text { on } U-f(U)\right\} .
$$

For $\sigma \in \mathscr{G}^{k}$ define $\phi_{k}(\sigma)$ exactly as before. Let us show that $\phi_{k}$ maps $\mathscr{G}^{k, \delta}$ into $\mathscr{G}^{k, \delta}$. Let $\left(x_{0}, y_{0}\right) \in V \backslash W^{s}(p)$, i.e. assume $x_{0} \neq 0$. Then take $g_{i} \in \mathscr{E}^{k}\left(x_{0}, y_{0}\right)$ with $\pi_{k, k-1}\left(g_{1}\right)=$ $\pi_{k, k-1}\left(g_{2}\right)$ and let $\left(x_{1}, y_{1}\right)=f\left(x_{0}, y_{0}\right)$. From lemma $7.1(\mathrm{a})$ for any $\varepsilon>0$ we can choose $V$ so small that:

$$
\begin{aligned}
\left|\Gamma_{k}\left(g_{1}\right)-\Gamma_{k}\left(g_{2}\right)\right|_{k, \delta} & =\left|\Gamma_{k}\left(g_{1}\right)-\Gamma_{k}\left(g_{2}\right)\right|_{k} \cdot\left\|x_{1}\right\|^{k-\delta} \\
\leq & \left(\frac{\left|\lambda_{1}\right|}{\left|\mu_{1}\right|^{k}}+\varepsilon\right)\left|g_{1}-g_{2}\right|_{k} \cdot\left\|x_{1}\right\|^{k-\delta} \\
& =\left(\frac{\left|\lambda_{1}\right|}{\left|\mu_{1}\right|^{k}}+\varepsilon\right)\left|g_{1}-g_{2}\right|_{k, \delta} \cdot\left(\frac{\left\|x_{1}\right\|}{\left\|x_{0}\right\|}\right)^{k-\delta} \\
& \leq\left(\frac{\left|\lambda_{1}\right|}{\left|\mu_{1}\right|^{k}}+\varepsilon\right) \cdot\left(\mu_{u}+\varepsilon\right)^{k-\delta} \cdot\left|g_{1}-g_{2}\right|_{k, \delta} .
\end{aligned}
$$

Furthermore $\left\|D f_{x}^{-1}\right\| \leq\left(\left(1 /\left|\lambda_{s}\right|\right)+\varepsilon\right)$. Now one can estimate the Lipschitz expansion:

$$
L\left(\phi_{k}\right) \leq L\left(\Gamma_{k}\right) \cdot\left\|D f_{x}^{-1}\right\| \leq\left(\frac{\left|\lambda_{1}\right|}{\left|\mu_{1}\right|^{k}}+\varepsilon\right) \cdot\left(\left|\mu_{u}\right|+\varepsilon\right)^{k-\delta}\left(\frac{1}{\left|\lambda_{s}\right|}+\varepsilon\right) .
$$


Since $\delta>0$ this last number is smaller than one, provided $\left(\left|\lambda_{1}\right| /\left|\lambda_{s}\right|\right) \cdot\left(\left|\mu_{u}\right|^{k-\delta} /\left|\mu_{1}\right|^{k}\right)<1$ and provided we take $\varepsilon>0$ small enough. It follows that $\phi_{k}$ maps $\mathscr{G}^{k, \delta}$ into $\mathscr{G}^{k, \delta}$ and moreover that this map is a contraction. Hence there is a unique fixed point $\sigma_{k}^{*}$ which extends $\sigma_{k}$. Statement (a) follows.

The proof of statement (b) goes exactly as the proof of theorem 7.3 if we replace $|\cdot|_{k}$ by $|\cdot|_{k, \delta}$ and use the estimates from above.

COROllary. Let $f: M \rightarrow M$ be a diffeomorphism with a saddle-point $p$ and let $\operatorname{dim} M=$ 2. Then one can extend a foliation $\mathscr{F}$ with tangencies along $W^{s}(p)$ (as above) to an invariant unstable foliation $\mathscr{F}^{*}$ for $p$, such that the $k$-jet along leaves is a $C^{1}$ function away from $W^{s}(p)$.

Proof. For the two-dimensional case the condition $\left(\left|\lambda_{1}\right| /\left|\lambda_{s}\right|\right) \cdot\left(\left|\mu_{u}\right|^{k-\delta} /\left|\mu_{1}\right|^{k}\right)<1$ is trivially satisfied. Therefore apply theorem 7.4.

Remark. As before $\mathscr{F}^{*}$ depends continuously on $f$ and $\mathscr{F}$.

Finally we wish to thank Freddy Dumortier and Anthony Manning for making many detailed suggestions after reading an earlier version of this paper.

\section{REFERENCES}

[Ha] P. Hartman. On local homeomorphisms of Euclidean spaces. Bol. Soc. Math. Mexicana 5 (1960).

[HP] M. Hirsch \& C. Pugh. Stable manifolds and hyperbolic sets. In Global Analysis. Proc. Symp. in Pure Math. XIV. Amer. Math. Soc. (1970), 133-164.

[Me1] W. de Melo. Structural stability of diffeomorphisms on two manifolds. Inv. Math. 21 (1973), 233-246.

[Me2] W de Melo. Moduli of stability of two-dimensional diffeomorphisms. Topology 19 (1980), 9-21.

[MP] W. de Melo \& J. Palis. Moduli of stability for diffeomorphisms. Lecture Notes in Math. 819. Springer-Verlag (1980), 315-339.

[MPS] W. de Melo, J. Palis \& S. J. van Strien. Characterizing diffeomorphisms with modulus of stability one. In Dynamical Systems and Turbulence, Warwick 1980. Springer Lecture Notes in Math. 898 (1981), 266-285.

[NPT] S. Newhouse, J. Palis \& F. Takens. Bifurcations and stability of families of diffeomorphisms. Publ. Math. Inst. Hautes Études Scientifiques 57 (1983).

[P1] J. Palis. On Morse-Smale dynamical systems. Topology 8 (1969), 385-404.

[P2] J. Palis. Moduli of stability and bifurcation theory. Proc. Int. Congress of Mathematicians Helsinki (1978).

[PS] J. Palis \& S. Smale. Structural stability theorems. In. Global Analysis. Proc. Symp. in Pure Math. XIV. Amer. Math. Soc. (1970), 223-232.

[R] C. Robinson. $C^{r}$ structural stability implies Kupka-Smale. In Dynamical Systems (ed. M. Peixoto) Academic Press (1973), 443-449.

[R1] J. Robbin. A structural stability theorem. Ann. of Math. 94 (1971), 447-493.

[R2] J. Robbin. Unfoldings of discrete dynamical systems. Ergod. Th. \& Dynam. Sys. 4 (1984), $421-486$

[Sm1] S. Smale. Differentiable dynamical systems, Bull. Amer. Math. Soc. 73 (1967), 747-817.

[Sm2] S. Smale. The $\Omega$-stability theorem. In Global Analysis. Proc. Symp. in Pure Math. XIV. Amer. Math. Soc. (1970), 289-297.

[S1] S.J.van Strien. Saddle connections of arcs of diffeomorphisms, moduli of stability. In Dynamical Systems and Turbulence, Warwick 1980. Springer Lecture Notes in Math. 898 (1981), 352-366.

[S2] S. J. van Strien. One parameter families of vectorfields. Bifurcations near saddle-connections. Ph.D. Thesis, Utrecht (1982). (An extended version will appear as a monograph.) 